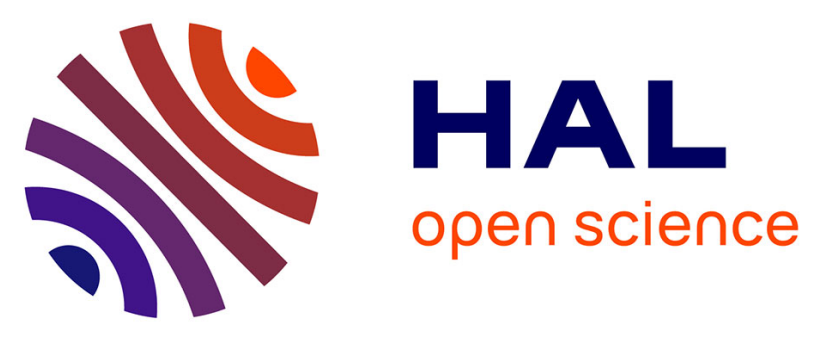

\title{
Chapitre II - L'étape de la crémation : les bûchers funéraires
}

\author{
Valérie Bel, Frédérique Blaizot, Christine Bonnet, Marie Gagnol, Patrice \\ Georges-Zimmermann, Jean-Luc Gisclon, Renaud Lisfranc, Anne Richier, \\ Alain Wittmann
}

\section{To cite this version:}

Valérie Bel, Frédérique Blaizot, Christine Bonnet, Marie Gagnol, Patrice Georges-Zimmermann, et al.. Chapitre II - L'étape de la crémation: les bûchers funéraires. Gallia - Archéologie de la France antique, 2009, Pratiques et espaces funéraires de la Gaule durant l'Antiquité, 66 (1), pp.89-150. 10.3406/galia.2009.3373 . hal-01911007

\section{HAL Id: hal-01911007 https://hal.science/hal-01911007}

Submitted on 7 Jan 2020

HAL is a multi-disciplinary open access archive for the deposit and dissemination of scientific research documents, whether they are published or not. The documents may come from teaching and research institutions in France or abroad, or from public or private research centers.
L'archive ouverte pluridisciplinaire HAL, est destinée au dépôt et à la diffusion de documents scientifiques de niveau recherche, publiés ou non, émanant des établissements d'enseignement et de recherche français ou étrangers, des laboratoires publics ou privés.

\section{(ㅇ)(1) $\$$}

Distributed under a Creative Commons Attribution - NonCommercial - NoDerivatives| 4.0 
Chapitre II

\title{
L'ÉTAPE DE LA GRÉMATION: LES BÛCHERS FUNÉRAIRES
}

\author{
Valérie BEL, Frédérique BlaIzOT, Christine BONNET, Marie-Émilie GAGNOL, \\ Patrice GeOrges, Jean-Luc Gisclon, Renaud Lisfranc, Anne Richier et Alain WittmanN
}

\section{NOMENCLATURE}

Dans la littérature archéologique, on rencontre de grandes difficultés pour identifier les structures de crémation souvent mal décrites par les auteurs. L'emploi des termes latins bustum et ustrinum, dont l'interprétation varie en outre énormément d'un archéologue à l'autre, complique d'emblée les faits. Réinterpréter les découvertes anciennes devient une véritable gageure ; cet ouvrage s'en fait l'écho à de multiples reprises.

Chez certains, ustrinum est employé pour désigner un bûcher établi au sol, chez d'autres un bûcher collectif, d'autres encore utilisent indifféremment bustum ou ustrinum à la place de bûcher, bustum étant également usité pour nommer les bûchers en fosse qui ont acquis le statut de sépulture (voir infra, chapitre IV, p. 183-186). Ainsi, lorsque la description d'une structure de crémation est insuffisamment précise, il est difficile de déterminer à quoi nous avons affaire. C'est pourquoi, nous proposons ici une typologie fondée sur des réalités archéologiques : bûcher au sol ou bûcher en fosse, bûcher collectif ou bûcher individuel. Nous profitons de cette mise au point pour rappeler que l'adjectif " primaire », employé pour désigner la structure de crémation stricto sensu, en l'occurrence les bûchers, doit se rapporter expressément à la structure et non pas à l'action de brûler. Il convient donc de préférer l'expression «structure primaire » à celle, elliptique et ambiguë, de «crémation primaire »(Blaizot, Tranoy, 2004). Enfin, il n'échappera pas que nous avons également pris soin d'employer le terme de "crémation ", qui est plus adapté à la réalité du produit fini que celui «d'incinération » qui signifie " réduit en cendres ", même si ce terme, employé au départ pour les déchets (Thomas, 1980), est passé par extension dans le vocabulaire funéraire de la communauté archéologique.

\section{LES DIFFÉRENTS TYPES DE BÛCHERS}

\section{BÛCHERS SUR LE SOL OU EN FOSSE}

Pour le centre et le sud de la Gaule (mais sans doute cette remarque pourrait-elle être étendue à l'ensemble des Gaules, voire du monde romain), les exemples de bûchers érigés sur le sol sont extrêmement rares au regard des dizaines d'exemplaires de bûchers en fosses reconnus à ce jour dans les ensembles funéraires du Sud-Est (voir infra, p. 99-103). On peut évoquer, en premier lieu, des raisons taphonomiques. Contrairement aux structures enfouies, les dispositifs de surface n'ont pas résisté à l'érosion des sols par les labours ou par les aménagements postérieurs, qui caractérisent la plupart des ensembles funéraires antiques. Il a été montré par ailleurs qu'un bûcher ponctuel aménagé sur le sol ne laissait que des traces peu perceptibles (Lambot, 1994, p. 256 ; Pautreau, 1994, p. 307). L'emplacement d'un tel bûcher ne porte pas toujours de marques de rubéfaction, dans la mesure où le sol est rapidement isolé de la chaleur de la crémation par les cendres qui s'accumulent à 
la base de l'édifice. Or, les seuls indices déterminants pour identifier une aire de crémation sont la rubéfaction ou plus largement, l'altération du sol sous l'effet de la chaleur et une répartition des restes osseux qui soit cohérente du point de vue anatomique.

Par ailleurs, les descriptions succinctes, sans documentation (photo)graphique, nous obligent à écarter des découvertes qui peuvent être - mais comment le savoir ? - des bûchers au sol mal conservés. Nous n'avons ainsi pas retenu le cas des Plaines à Cayrac (Tarn-et-Garonne) où, dans le secteur le plus densément occupé par les tombes, sont mentionnées diverses accumulations de charbons de bois et de mobilier calciné. En effet, l'argumentation fait défaut et, au regard de la diversité des pratiques potentielles relevées dans les ensembles funéraires en général et dans l'environnement immédiat des sépultures en particulier, il nous semble préférable de ne pas retenir l'hypothèse d'un bûcher, même si l'auteur a voulu reconnaître dans ces vestiges "un lieu privilégié de crémation ». De même, sur le site de la station de métro François-Verdier à Toulouse (Haute-Garonne), la présence sur le niveau de sol d'une zone rubéfiée qui conserve les restes d'une planche associée à une monnaie non brûlée du $\mathrm{II}^{\mathrm{e}} \mathrm{s}$. apr. J.-C. ne peut être mise en rapport, malgré les hypothèses avancées, avec un bûcher à même le sol.

Quand l'argumentation n'est pas très développée, la forme et les dimensions de la structure recelant des couches charbonneuses et des os sont parfois des critères suffisants pour déterminer un bûcher, mais il n'est généralement pas possible d'en préciser la nature : sur le sol ou en fosse. Dans l'ensemble funéraire de l'Érable à Gièvres (Loir-et-Cher), qui a fait l'objet de plusieurs campagnes archéologiques au $\mathrm{XIX}^{\mathrm{e}}$ s. et au début du XX $\mathrm{XX}^{\mathrm{e}}$ s. (Jollois, 1830), deux structures plus ou moins rectangulaires furent observées et fouillées en 1823, dont l'une mesurait 2,25 m à 2,60 m de longueur, sur 1,30 m à 1,60 m de largeur. Elles étaient couvertes d'un " amas de cendre », de charbons de bois et d'os calcinés. Ces structures sont désignées par le terme d'ustrina, et force est aujourd'hui de constater que les informations essentielles manquent.

Pour ce qui concerne le bûcher construit de l'ensemble funéraire des Vernes à Faverdines (Cher), les arguments sont indirects. Ce petit édifice de pierres, mesurant 4,40 $\mathrm{m}$ x 3,90 $\mathrm{m}$ et dont il ne subsiste que les fondations (blocage de moellons calcaires déposé sur de grosses dalles calcaires), a été considéré comme étant la base d'un bûcher funéraire en raison des vestiges (tessons, verre, os, charbons de bois...) retrouvés dans le fossé qui borde cette structure. Le pendage de la couche a montré que les débris constituant l'amas de vidange ont été jetés depuis l'édicule (Fourteau-Bardaji et al., 1993, p. 268). D’autres bûchers construits ont été exhumés en région Centre, comme aux Mahis à Gy-en-Sologne ; ils étaient très mal conservés et si les murs qui évoquent des structures pérennes n'avaient subsisté, il n'aurait pas été possible de déterminer la nature de ces vestiges.

La question des « enclos » relève en partie de la même problématique. Elle montre en tout cas qu'il n'est pas si simple d'identifier un bûcher, même construit. Par leur description, deux structures de la Vayssière à L'Hospitaletdu-Larzac (Aveyron), dont l'une est dénommée « enclos funéraire T25 » (Vernhet, 1987b, p. 131), ne diffèrent pas de bûchers permanents et construits. Elles possèdent des murs bâtis de pierres sèches ; l'un est un carré de 3,10 m de côté et l'autre forme un rectangle de 4,32 m x 1,95 m. L'élément suggestif est représenté par les $30 \mathrm{~kg}$ de charbons de bois exhumés de l'un d'entre eux.

Les mentions d'amas de résidus charbonneux ou de couches de cendres en lits superposés sont fréquentes en contexte funéraire à l'époque romaine : à Vaison-laRomaine, dans le Vaucluse (Bel, Meffre, 1991, p. 115-121), à Carpentras, dans le Vaucluse (fouille inédite : D. Carru), à Cimiez, à Nice, dans les Alpes-Maritimes (Mouchot, 1966, p. 28), à Castelnau-le-Lez, dans l'Hérault (Ramonat, Sahuc, 1988, p. 156-161), ou à Roanne (Vaginay, 1983). À Cimiez, D. Mouchot mentionne l'existence de couches de combustion très riches en fioles de verre brisées ou fondues alternées avec des couches d'argile vierge battue. À Vaisonla-Romaine, un sondage réalisé par J.-C. Meffre dans le quartier des Roussillons (Bel, Meffre, 1991, p. 115-121) a recoupé, au pied d'un abrupt rocheux, une structure d'une surface d'environ $30 \mathrm{~m}^{2}$, constituée de deux couches de sédiment très charbonneux (de $0,15 \mathrm{~m}$ et $0,60 \mathrm{~m}$ d'épaisseur maximale). Celles-ci comportent des ossements humains, des pierres et des éléments de mobilier brûlés, datés du dernier tiers du $\mathrm{I}^{\mathrm{er}} \mathrm{s}$. et du premier tiers du $\mathrm{II}^{\mathrm{e}} \mathrm{s}$. Les deux couches de résidus sont séparées par un amas de pierres brûlées de $0,30 \mathrm{~m}$ d'épaisseur et reposent sur le substrat non rubéfié. Des parties de squelettes non brûlés en connexion anatomique (mandibule et segment de rachis cervical ; fémurs, os coxaux et sacrum ; métatarsiens), et des os isolés ont été observés au sein de la couche charbonneuse supérieure, avec des traces de carbonisation partielle sur certaines pièces, interprétées comme l'expression d'une carbonisation incomplète. Sur le site de Navitau à Castelnaule-Lez, la structure désignée comme un ustrinum, observée 
dans un sondage, est également constituée de deux couches charbonneuses (d'environ 0,10 $\mathrm{m}$ et $0,05 \mathrm{~m}$ d'épaisseur) séparées par une mince couche de sédiment stérile et par une dalle de calcaire. Cette dernière présente, comme le substrat rocheux sous-jacent au dépôt, des traces d'une exposition au feu (Ramonat, Sahuc, 1988, p. 156). À la différence des dépôts observés à Vaison-la-Romaine, la couche charbonneuse inférieure de Navitau n'a livré aucun os brûlé et la couche supérieure, seulement trois fragments. Ces deux niveaux renfermaient en revanche une très grande quantité de fragments de céramique brûlée ( ${ }^{\mathrm{er}}$ s. apr. J.-C.) et de rares ossements de faune non brûlés. Ces dépôts occupent une dépression dans le substrat, en partie comblée par des occupations antérieures à l'époque romaine, observée dans un sondage de $1,30 \mathrm{~m}$ sur $0,45 \mathrm{~m}$. Dans ce cas, la faible quantité d'ossements brûlés pourrait faire penser que la structure ne conservait que le seul matériel du banquet funéraire, mais l'hypothèse nécessiterait, pour être validée, une analyse précise des types fonctionnels (voir infra, p. 62-71). À Roanne (Loire), dans un talweg ont été observées deux couches de " terre noire cendreuse » avec des fragments de céramique et d'os brûlés, séparées par un niveau de cailloutis.

Globalement, en l'absence de fouille extensive et d'une étude de la répartition des ossements humains brûlés, il est difficile de se prononcer sur la nature de ces différentes structures : bûchers en place, couches d'occupation piégées dans des creux, dépositions secondaires de produits de crémation, ou encore rejets d'artefacts liés aux repas funéraires, comme le suggère l'exemple de Navitau.

Des aires de crémation de surface sont en revanche bien attestées à Marseille (Bouches-du-Rhône). Il s'agit d'une aire rubéfiée quadrangulaire de 2,50 m sur 2,10 m, dépourvue de restes de crémation, reconnue dans l'ensemble funéraire romain de Sainte-Barbe (Moliner et al., 2003, p. 43), et d'un bûcher aménagé sur le rocher aplani et profondément calciné, au bassin de Carénage (Toulouzan, 1831, cité par Moliner et al., 2003, p. 44). La situation de la zone rubéfiée de Sainte-Barbe, cernée par des dépôts secondaires de crémation évoque une aire de crémation commune à plusieurs sépultures (Moliner et al., 2003, p. 44). Lorsque la surface rubéfiée n'est plus associée à des résidus d'ustion, si ce n'est quelques fragments de charbons de bois, on ne peut déterminer si elle se rapporte à une aire de crémation ou plutôt à un foyer aménagé pour les besoins des rites accompagnant les funérailles ou des pratiques liées au culte de la mémoire. Cette dernière interprétation est proposée pour les traces ténues de rubéfaction et les petites concentrations de charbons de bois relevées sur le sol de l'ensemble funéraire de la ZAC Sextius-Mirabeau à Aix-enProvence (Nin et al., 2006, p. 114-115).

Il existe également un exemple de bûcher établi à l'intérieur d'une construction maçonnée préexistante. Il s'agit d'un petit enclos ou mausolée quadrangulaire de 3,50 m de largeur interne, constitué en façade de blocs de grand appareil, mis au jour sur le site des Combes, à proximité de l'agglomération secondaire de Murviel-lès-Montpellier (Schwaller, 1992 ; Gallia Informations, 1992, p. 147-148 ; Richard, Gascou, 1992, p. 443-445). Dans une deuxième phase d'utilisation, la construction a été comblée par des résidus provenant de crémations datées du milieu du $\mathrm{I}^{\mathrm{er}} \mathrm{s}$. apr. J.-C., avant d'être réaménagée pour servir d'aire de crémation dans le courant du troisième quart du $\mathrm{I}^{\mathrm{er}} \mathrm{s}$. apr. J.-C. Cette réutilisation a été identifiée notamment grâce aux traces de rubéfaction observées sur les parements internes.

Mis à part les aires de crémation au sol exhumées dans le quartier de Vaise, sur les sites de la voie de l'Océan et de Villa Montel, aucune autre n'a été décrite pour Lyon ou la région Rhône-Alpes, où les bûchers en fosse sont en revanche très fréquents. Les informations relatives au bûcher de Villa Montel sont malheureusement succinctes : sa surface n'est pas précisée, ni sa morphologie ; conservé sur une épaisseur de $50 \mathrm{~cm}$, il se compose de quatre niveaux charbonneux irréguliers, parsemés d'esquilles osseuses et de tessons de céramique calcinée, séparés par des sables plus ou moins grossiers relatifs à des épisodes de débordement d'un talweg. Aucune datation du matériel n'a été réalisée. Les bûchers établis en bordure de la voie de l'Océan sont illustrés par 43 zones rubéfiées de superficie variable dont les plus grandes atteignent $10 \mathrm{~m}^{2}$ (Frascone, 1999 , p. 127-130). Un tiers ne contiennent plus aucun artefact ni os et les $3 / 4$ d'entre eux renferment moins de $50 \mathrm{~g}$ d'os. La stratigraphie révèle des niveaux successifs et dans un cas au moins, le NMI s'élève à trois individus tandis que plusieurs collages entre les céramiques provenant de différentes structures de dépôt attestent l'emploi collectif de ces aires.

En Auvergne, les mentions de bûchers sont très peu nombreuses, et l'imprécision des descriptions ne permet pas, le plus souvent, d'en définir la nature. C'est le cas par exemple, des ensembles de Voingt (Puy-de-Dôme) et de Chemilly (Allier), mentionnés sous la forme de « couches de cendres » riches en artefacts calcinés. Les surfaces occupées par ces structures sont très importantes : jusqu'à $20 \mathrm{~m}$ sur $2 \mathrm{~m}$ à $3 \mathrm{~m}$ de largeur pour Voingt. On ne compte 


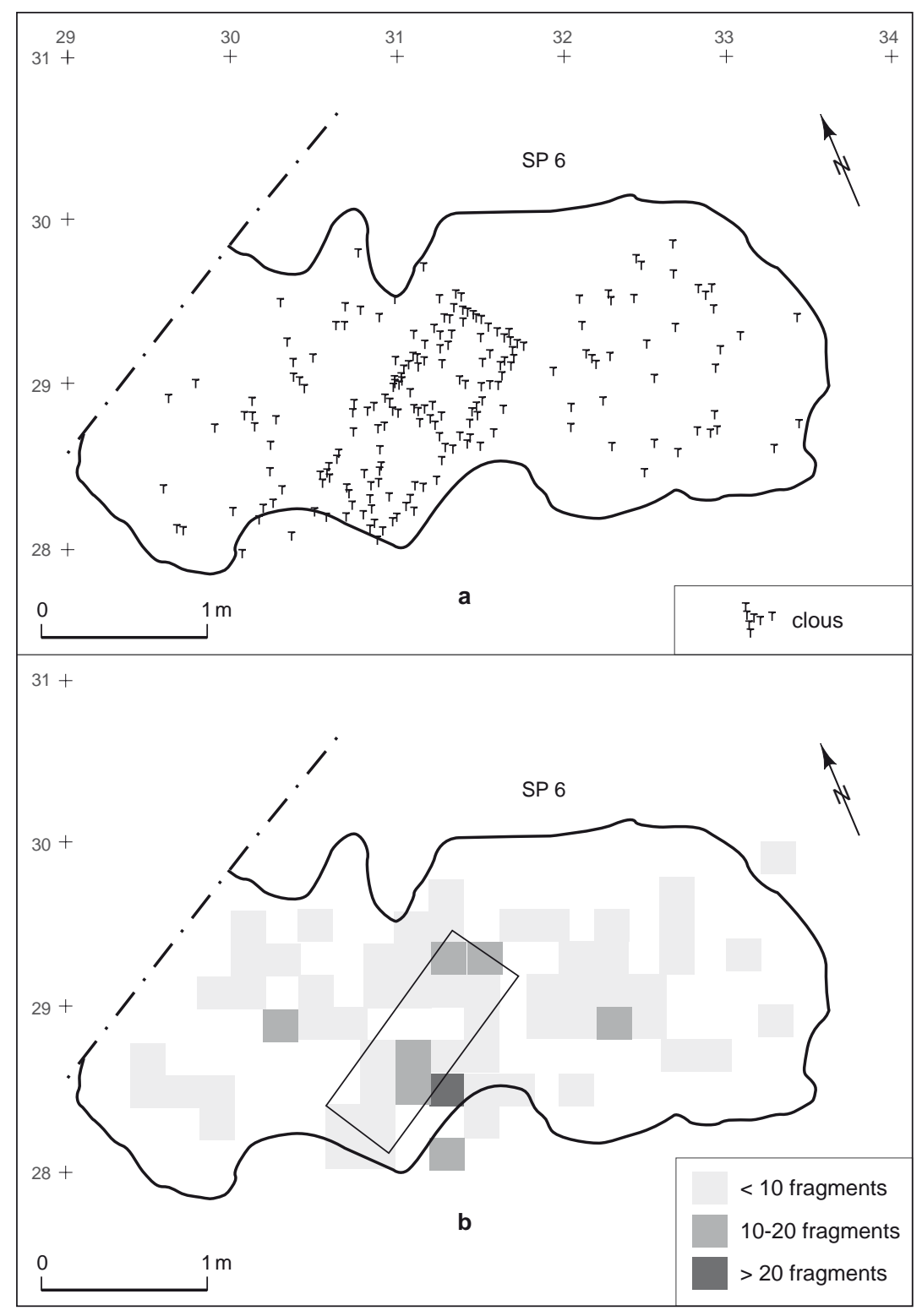

Fig. 72 - Aire de crémation et bûcher 6 du Pâtural à Clermont-Ferrand, Puy-de-Dôme :

$\boldsymbol{a}$, répartition des clous ; $\boldsymbol{b}$, répartition des fragments osseux (relevé : C. Gouzel ; DAO : G. Macabéo, Inrap).

en tout cas, aucun bûcher construit dans toute la région. Un bûcher au sol daté de la première moitié du $\mathrm{I}^{\mathrm{er}} \mathrm{s}$. est attesté sur le site du Pâtural à Clermont-Ferrand (Gouzel, 1996, p. 47-51). Il s'agit d'une nappe de sédiment très charbonneux, riche en mobilier, en esquilles osseuses et clous calcinés (fig. 72). L'ensemble se répartit sur une surface de $10 \mathrm{~m}^{2}$ globalement rectangulaire aux contours irréguliers. Au centre, une centaine de clous en fer dessine le négatif d'un rectangle de $0,50 \mathrm{~m}$ sur $1,50 \mathrm{~m}$, qui correspond probablement au dispositif du corps (lit ?), tandis que de part et d'autre, de grands amas de clous désorganisés sont relevés. L'étude des ossements, récemment réalisée (Pélissier, 2008), n'a malheureusement guère apporté d'informations : les $3 / 4$ des restes osseux n'ont pas été retrouvés et la documentation s'est avérée très confuse. L'importante dispersion des vestiges humains, toutes régions anatomiques confondues, incite l'auteur à envisager que l'aire de crémation fut utilisée à plusieurs reprises. Les trente-deux vases en NMI n'offrent cependant pas d'argument à l'hypothèse de plusieurs crémations, puisque sur le même site, des dépôts 
de résidus en fosse comportent plus d'une vingtaine, voire une trentaine de vases en NMI.

À l'heure actuelle, rares sont les petits bûchers en fosse identifiés pour l'époque romaine en Auvergne : trois à la Grande Borne, un à la Buyre à Chappes (Puy-deDôme), cinq à Pont-du-Château et un probable exhumé il y a quelques années à la Pardieu, à Clermont-Ferrand. Une structure, fouillée au XIX ${ }^{\mathrm{e}} \mathrm{s}$. à Bromont-Lamothe, à Riom (Puy-de-Dôme), traditionnellement désignée sous le terme d'ustrinum, se présente sous la forme d'un grand rectangle excavé, de 15 m sur 4 m (Mondanel, 1982, p. 18).

Le site de la caserne Niel à Toulouse (fouilles de 2004) a livré un bûcher construit, dont il ne subsistait plus que la partie basse constituée de briques de terre cuite (us 102) et d'une file de briques liées au mortier de chaux (us 101). Le bâti de briques (module moyen : 0,34 m x 0,20 m x 0,03 m) mesure $1,13 \mathrm{~m}$ de longueur et $0,70 \mathrm{~m}$ de largeur. Un espace rectangulaire $(0,72 \mathrm{~m}$ sur $0,28 \mathrm{~m})$ est délimité par une couche de mortier qui, à l'origine, était surmontée au moins d'une assise de briques. La fonction de bûcher a été établie en raison de la couleur de l'épiderme du mortier du parement interne, rouge à rosé, typique d'une rubéfaction. Dans la masse du mur, ce même mortier a en revanche conservé sa teinte gris-blanc.

Dans le sud-est de la Gaule, les rares exemples de bûchers de surface ne permettent guère de se faire une idée de la place réelle de ce type d'aménagements, dans les ensembles funéraires de l'époque romaine. La documentation disponible pour le second âge du Fer est encore plus réduite, mais en Languedoc, deux découvertes témoignent de leur existence. Il s'agit de l'aire de crémation datée de la fin du III ${ }^{\mathrm{e}}$ s. av. J.-C., fouillée par B. Dedet au sein de l'espace funéraire mis au jour au pied de l'oppidum d'Ambrussum à Villetelle, dans l'Hérault (Fiches dir., 1989, p. 39 ; Fiches, Py, 2002 et 2003, p. 394), et de celle, contemporaine, découverte sur l'oppidum de Puech Maho à Sigean, dans l'Aude (Dedet, Schwaller, 1990, p. 141 ; Gailledrat, Belarte, 2002, p. 600). À la même époque, il existe aussi des structures de crémations en fosse, mais elles semblent moins répandues qu'elles le seront au Haut-Empire dans les mêmes régions (voir infra, p. 99-103). L'aire de crémation du III ${ }^{\mathrm{e}}$ s. av. J.-C. étudiée sur l'oppidum d'Ensérune à Nissan-lez-Ensérune, dans l'Hérault, d'une superficie minimale de $15 \mathrm{~m}^{2}$, a été aménagée sur le substrat, après enlèvement de la couverture pédologique (Jallet et al., 1998, p. 203-205). Sur l'aire funéraire hellénistique de Sainte-Barbe à Marseille, apparaissent deux exemples de bûchers individuels en fosse (IV -III $^{\mathrm{e}}$ s. av. J.-C.). Il s'agit d'excavations rubéfiées de forme allongée, associées à quelques charbons de bois ou de rares esquilles osseuses et dont l'une, longue de 1,85 m et large de $0,48 \mathrm{~m}$, a été recoupée par un loculus contenant un vase ossuaire (Moliner et al., 2003, p. 35, p. 257 et p. 272). Dans les régions Rhône-Alpes et Auvergne, les bûchers laténiens sont inconnus, mis à part ceux des ensembles aristocratiques de Bel Air à Saint-Laurent-la-Conche dans la Loire et de la Cime des Bruyères à Pulvérières dans le Puy-de-Dôme, datés $d u I^{e}$ s. ; il s'agit de bûchers en fosse. Sur le site d'Aulnat/Gandaillat, deux grandes dépressions comblées de plusieurs couches de résidus de crémations séparées par des sédiments de ruissellement pourraient témoigner, sinon d'aires de crémation in situ, d'une accumulation de résidus provenant de bûchers établis au sol. Le mobilier qu'elles contiennent permet de les dater de la fin du $\mathrm{III}^{\mathrm{e}} \mathrm{s}$. et du $\mathrm{II}^{\mathrm{e}} \mathrm{s}$. En Midi-Pyrénées, on ne dispose d'aucune description fiable pouvant se rapporter sans ambiguïté à un bûcher, ni même à toute structure liée à la crémation ; par exemple dans le Gers, toutes les structures dites «funéraires » du second âge du Fer sont en fait des « puits » dont le caractère funéraire reste à démontrer (Lapart, Petit, 1993 ; Verdin, Vidal, 2004 ; Verdun, Bardot, 2007), tandis que dans le Tarn, en dépit de ce qu'en dit la Carte archéologique de la Gaule (« au I ${ }^{\mathrm{er}} \mathrm{s}$. av. J.-C. l'incinération en fosse est la pratique funéraire la plus usitée », $c f$. Provost dir., 1995, p. 49), les seuls exemples relevés, comme celui de Montans, se rapportent à des récipients enterrés et associés à des dépôts de vases, mais pour lesquels aucun vestige osseux n'est mentionné (CERAM, 1987, p. 69).

L'utilisation de bûchers en fosse apparaît donc exceptionnelle et marginale avant la période augustéenne. En revanche, le Haut-Empire est caractérisé, dans les régions considérées, par le développement de structures excavées de forme oblongue et souvent rectangulaire, qui présentent des traces de crémation sur place, soit parce que le bûcher a été installé dans la fosse elle-même, soit parce qu'il a été établi juste au-dessus. Souvent bien conservées, ces fosses permettent d'approcher dans d'assez bonnes conditions les modalités crématoires, le mode de construction du bûcher, la disposition du sujet et du mobilier livrés aux flammes et les différentes interventions ayant pu être réalisées pendant ou après la crémation sur l'aire crématoire. La multiplication des découvertes récentes et les progrès méthodologiques permettent à la fois de mieux cerner les modalités de diffusion des bûchers en fosse au cours des deux premiers siècles de notre ère mais aussi de mettre en évidence la diversité des formes et des usages (Bel, Tranoy, 1993b ; Blaizot, Tranoy, 2004 ; Bel et al., 2008). 


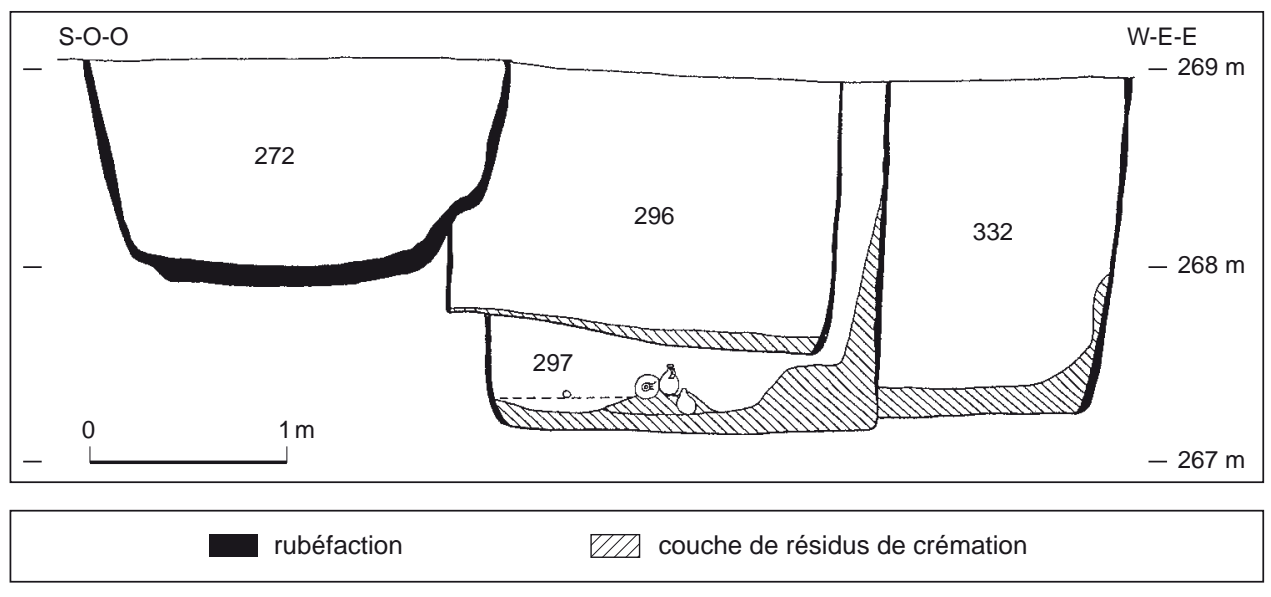

Fig. 73 - Bûchers en fosse de la rue de la Favorite à Lyon (relevé : L. Tranoy, Université de La Rochelle ; DAO : G. Macabéo, Inrap).

\section{GRITÈRES D'IDENTIFICATION DES BÛCHERS EN FOSSE}

Le premier indice en faveur d'une utilisation de la fosse pour la crémation est la rubéfaction des parois et/ou du fond et, plus largement, d'une altération sous l'effet de la chaleur. Cette rubéfaction est souvent superficielle ou marquée sur moins de 0,04 m d'épaisseur. Toutefois, dans le cas du bûcher du Larsou à Réalville (Tarn-et Garonne) (fin du $\mathrm{I}^{\mathrm{er}}$-début du $\mathrm{II}^{\mathrm{e}} \mathrm{s}$. de notre ère), la rubéfaction des parois argileuses a été reconnue sur une épaisseur de 0,27 m (Rigal, 1996, p. 22). La coloration des parois n'est pas toujours homogène au sein d'une même structure. Par exemple, dans le bûcher de Gallière à Montpellier - une fosse de 1,65 m de longueur et 0,85 m de largeur, au grand axe orienté nord-sud -, seules les parois nord et ouest ainsi que le fond étaient rubéfiés. Généralement, du fait qu'il s'agit d'une structure excavée, la rubéfaction ne se produit que sur les parties hautes de la fosse (milieu oxydant) qui ne sont pas toujours conservées. En conséquence, dans les ensembles funéraires d'époque romaine, la fonction de bûcher est le plus souvent suggérée par d'autres indices, comme la morphologie et le comblement des fosses.

Les fosses à parois rubéfiées sont en effet de plan allongé, à angles droits ou arrondis (fig. 73), souvent avec un fond plat et des parois verticales ou subverticales, à l'instar des fosses réalisées pour les inhumations. Dans les régions étudiées dans cet ouvrage, le fond est plat, parfois en auge. Ainsi, dans leur partie profonde, ces bûchers ne sont guère plus étroits qu'à l'ouverture ; on ne connaît aucun exemplaire dont le profil transversal accuse un « V » prononcé, à l'image de ceux de Krefeld-Gellep en Rhénanie (Pirling, 2002, p. 496-498). Les structures de crémation de forme circulaire, qualifiées de rares à Krefeld-Gellep (id., ibid., p. 492) sont exceptionnelles dans nos séries. On peut mentionner la fosse de crémation 155 découverte dans l'enclos 2 de la ZAC Sextius-Mirabeau à Aix-en-Provence (Nin et al., 2006, p. 53-54). Celle-ci, au profil évasé, est quadrangulaire à la base $(0,70 \mathrm{~m}$ x $0,30 \mathrm{~m})$ et circulaire au sommet (diamètre $1,05 \mathrm{~m}$; profondeur : $0,60 \mathrm{~m}$ ). Sur le même site, dans l'enclos 3, une aire de crémation a été aménagée dans une fosse préexistante et partiellement comblée, peu profonde et de forme irrégulière (structure 40). La zone rubéfiée, limitée sur un côté par une banquette en terre, dessine une aire vaguement semi-circulaire. Un autre exemple de bûcher en fosse de forme circulaire ( $1 \mathrm{~m}$ de diamètre), conservé sur $0,17 \mathrm{~m}$ de profondeur seulement, a été mis au jour dans l'ensemble rural de l'Estel à Vers-Pont-du-Gard. Le bûcher de Champ Chalatras, aux Martres-d'Artière, est de plan carré ; un seul bûcher sur le site de la Favorite à Lyon affecte cette forme (bûcher 249), également relevée à Krefeld-Gellep, dans un très petit nombre de cas.

La taille ne constitue pas un critère déterminant. La variabilité des modules s'observe parfois au sein d'un même ensemble ou pour une même agglomération, comme à Nîmes ou à Aix-en-Provence (fig. 74). Des variations apparaissent également entre les différents sites ou secteurs géographiques. Ainsi, parmi les séries les mieux documentées, Nîmes et les Communaux de Saint-Cézaire à Vernègues fournissent des valeurs plus importantes qu'à Aix, Marseille ou Saint-Paul-Trois-Châteaux. C'est ce dernier ensemble 


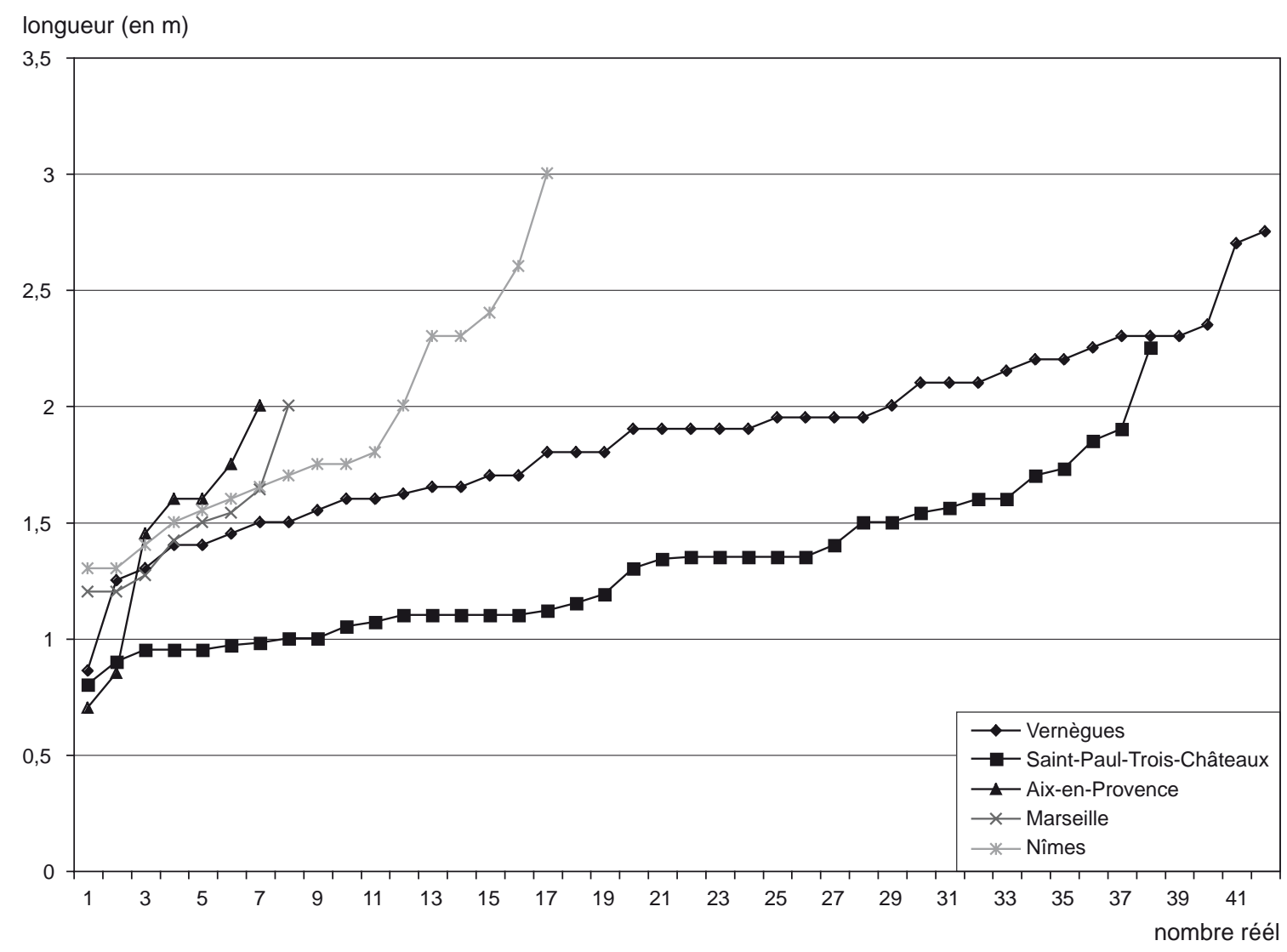

Fig. 74 - Longueurs des bûchers complets relevées dans les ensembles de Vernègues (les Communaux de Saint-Cézaire), Saint-Paul-Trois-Châteaux (le Valladas), Aix-en-Provence (ZAC Sextius-Mirabeau) et Marseille (Sainte-Barbe) (DAO : V. Bel, Inrap).

qui fournit les bûchers avec les valeurs les plus faibles. Dans ce cas, les rares données anthropologiques disponibles et la nature du mobilier associé montrent que la taille réduite des fosses n'est pas due à l'âge au décès des sujets qui y ont été brûlés : si certaines fosses de taille réduite ont été utilisées pour des sujets immatures, d'autres sont en revanche attribuées à des adultes (Bel et al., 2002, p. 89). Les données lyonnaises montrent que la variabilité au sein de chaque ensemble peut être équivalente, voire légèrement supérieure à la variabilité entre les sites (tabl. VIII). L'ensemble funéraire de la Favorite est celui qui présente la plus grande variabilité, sans doute parce que c'est celui pour lequel les données sont les plus nombreuses. La longueur des 73 bûchers complets est de 0,66 m à 3,05 m avec une moyenne de 2,01 m et un écart-type de 3,60, tandis que si l'on réunit tous les bûchers de Lyon (onze sites utilisables), les valeurs extrêmes oscillent entre $0,60 \mathrm{~m}$ et $3,05 \mathrm{~m}$ pour une moyenne de $1,50 \mathrm{~m}$ et un écart-type de 3,05. Il est évident que la série de la Favorite, qui se caractérise par la présence de grands bûchers, donne une image un peu parti- culière du phénomène, car sur les autres sites, les moyennes varient de 1,10 m à 1,20 m et l'écart-type de 0,18 à 0,54.

L'inégalité du corpus disponible conduit à nuancer l'hypothèse précédemment émise d'une diminution de la taille des bûchers à partir du troisième quart du $\mathrm{I}^{\mathrm{er}} \mathrm{s}$. (Bel, Tranoy, 1993b, p. 99). En effet, l'effectif des tombes anciennes (7 exemplaires dans le Midi et la vallée du Rhône sans compter celui de Villelongue-d'Aude, dans l'Aude et 20 exemplaires à la Favorite à Lyon) est très faible. Dans le Midi et la vallée du Rhône la moyenne des données et surtout la valeur minimale des longueurs des fosses datées de la première moitié $d u \mathrm{I}^{\mathrm{er}} \mathrm{s}$. apparaissent légèrement supérieures à celles des bûchers postérieurs au milieu du $\mathrm{I}^{\mathrm{er}} \mathrm{s}$., mais la variabilité est telle que les différences pourraient être simplement dues à l'état de notre documentation (fig. 75). Si l'on reprend les données brutes, on comprend que le poids du site de la Favorite a été sensible dans l'interprétation des données qui a été réalisée en 1993 : la longueur moyenne des bûchers, qui est de 2,16 m pour la première moitié du $\mathrm{I}^{\mathrm{er}} \mathrm{s}$. (min. 1,10 m, max. 3,05 m, écart-type de 0,41) passe à 


\begin{tabular}{|c|c|c|c|c|}
\hline Localisation & $\mathbf{N}^{\circ}$ bûcher & Datation & NMI & $\begin{array}{c}\text { Taille } \\
\text { (en m) }\end{array}$ \\
\hline Rue des Granges & 17 & milieu $\mathrm{er}^{\mathrm{er}} \mathrm{s}$. & 1 & $1,7 \times 1,6$ \\
\hline Rue des Granges & 18 & milieu $\mathrm{er}^{\mathrm{er}} \mathrm{s}$. & 1 & $1,6 \times 1,1$ \\
\hline Rue des Granges & 22 & milieu $\mathrm{ler}^{\mathrm{er}} \mathrm{s}$. & 1 & $1,3 \times 0,9$ \\
\hline Rue des Granges & 36 & milieu $\mathrm{er}^{\mathrm{er}} \mathrm{s}$. & 1 & $1,4 \times 0,95$ \\
\hline Rue des Granges & 45 & milieu $\mathrm{I}^{\mathrm{er}} \mathrm{s}$. & 1 & $1,65 \times 1,2$ \\
\hline Rue des Granges & 46 & milieu $\mathrm{ler}^{\mathrm{er}} \mathrm{s}$. & 1 & $1,3 \times 0,7$ \\
\hline Rue des Granges & 48 & milieu $\mathrm{l}^{\mathrm{er}} \mathrm{s}$. & 1 & $1,3 \times 0,8$ \\
\hline Rue du Commandant-Charcot & Les Roses, 1 & Augustéen & 1 & $1 \times 1$ \\
\hline Rue du Commandant-Charcot & Les Roses, 25 & Augustéen & 1 & $1 \times 0,75$ \\
\hline Avenue du Point-du-Jour & 1000 & fin $I^{\mathrm{er}}$-début $\mathrm{II}^{\mathrm{e}} \mathrm{s}$. & 1 & $2 \times 0,90$ \\
\hline 77-79, rue Pierre-Audry & 3041 & $2^{\mathrm{e}}$ quart Ier $\mathrm{s}$. & 1 & $2,10 \times 1,40$ \\
\hline 77-79, rue Pierre-Audry & 59 & $2^{\mathrm{e}}$ moitié $\mathrm{l}^{\mathrm{er}} \mathrm{s}$. & 2 & $0,80 \times 1$ \\
\hline 77-79, rue Pierre-Audry & 150 & $\mathrm{II}^{\mathrm{e}}-\mathrm{II}^{\mathrm{e}} \mathrm{s}$ & 1 & $0,60 \times 0,42$ \\
\hline 77-79, rue Pierre-Audry & 159 & $\| I^{e}-I I^{e} s$ & 1 & $0,70 \times 0,55$ \\
\hline 77-79, rue Pierre-Audry & 1093 & $\| I^{e}-I I I^{e} s$. & 1 & $0,80 \times 0,66$ \\
\hline 54, rue Pierre-Audry & 22 & $2^{\mathrm{e}}$ moitié $\mathrm{I}^{\mathrm{er}} \mathrm{s}$.-début II $\mathrm{s}$. & 1 & $1,35 \times 0,75$ \\
\hline 54, rue Pierre-Audry & 30 & fin Ier s.-début II $\mathrm{s}$. & 1 & $1,55 \times 0,70$ \\
\hline 54, rue Pierre-Audry & 54 & non daté & 1 & $1,55 \times 0,60$ \\
\hline 54, rue Pierre-Audry & 56 & fin ler s.-début II s. & 1 & $1,30 \times 0,70$ \\
\hline 54, rue Pierre-Audry & 58 & $\mathrm{Il}^{\mathrm{e}} \mathrm{s}$ & 2 & $1,75 \times 0,55$ \\
\hline Rue de la Favorite & 243 & $1^{\text {re }}$ moitié $1^{\text {er }} \mathrm{s}$. & 1 & 2,2 \\
\hline Rue de la Favorite & 396 & $1^{\text {re }}$ moitié $1^{\text {er }} \mathrm{s}$. & 1 & 2,25 \\
\hline Rue de la Favorite & 255 & $1^{\mathrm{re}}$ moitié $\mathrm{l}^{\mathrm{er}} \mathrm{s}$. & 1 & 2,3 \\
\hline Rue de la Favorite & 405 & $1^{\text {re }}$ moitié $1^{\text {er }} \mathrm{s}$. & 1 & 2,25 \\
\hline Rue de la Favorite & 114 & $1^{\text {re }}$ moitié $~^{\text {er }} \mathrm{s}$. & 1 & 2 \\
\hline Rue de la Favorite & 299 & $2^{\mathrm{e}}$ moitié $\mathrm{I}^{\mathrm{er}} \mathrm{s}$.-début $\mathrm{II}^{\mathrm{e}} \mathrm{s}$. & 1 & 1,75 \\
\hline Rue de la Favorite & 80 & $2^{\mathrm{e}}$ moitié $\mathrm{I}^{\mathrm{er}} \mathrm{s}$.-début II $\mathrm{s}$. & 2 & $?$ \\
\hline Rue de la Favorite & 199 & $2^{\mathrm{e}}$ moitié $\mathrm{I}^{\mathrm{er}} \mathrm{s}$ - -début $\mathrm{II}^{\mathrm{e}} \mathrm{s}$. & 1 & $?$ \\
\hline Rue de la Favorite & 131 & $2^{\mathrm{e}}$ moitié $\mathrm{I}^{\mathrm{er}} \mathrm{s}$.-début $\mathrm{II}^{\mathrm{e}} \mathrm{s}$. & 1 & 0,93 \\
\hline Rue de la Favorite & 76 & $2^{\mathrm{e}}$ moitié $\mathrm{I}^{\mathrm{er}} \mathrm{s}$.-début $\|^{\mathrm{e}} \mathrm{s}$. & 1 & $?$ \\
\hline Rue de la Favorite & 35 & $2^{\mathrm{e}}$ moitié $\mathrm{I}^{\mathrm{er}} \mathrm{s}$ - -début II $\mathrm{s}$. & 1 & 1 \\
\hline Rue de la Favorite & 281 & $2^{\mathrm{e}}$ moitié $\mathrm{l}^{\mathrm{er}} \mathrm{s}$.-début $\|^{\mathrm{e}} \mathrm{s}$. & 1 & 1,15 \\
\hline Rue de la Favorite & 328 & $2^{\mathrm{e}}$ moitié $\mathrm{I}^{\mathrm{er}} \mathrm{s}$.-début $\mathrm{Il}^{\mathrm{e}} \mathrm{s}$. & 1 & 1,3 \\
\hline Rue de la Favorite & 7 & $2^{\mathrm{e}}$ moitié $\mathrm{I}^{\mathrm{er}} \mathrm{s}$ - -début $\mathrm{II}^{\mathrm{e}} \mathrm{s}$. & 1 & 0,66 \\
\hline Rue de la Favorite & 98 & $2^{\mathrm{e}}$ moitié $\mathrm{I}^{\mathrm{er}} \mathrm{s}$.-début $\mathrm{Il}^{\mathrm{e}} \mathrm{s}$. & 1 & 1,3 \\
\hline Rue de la Favorite & 31 & $2^{\mathrm{e}}$ moitié $\mathrm{I}^{\mathrm{er}} \mathrm{s}$.-début II $\mathrm{s}$. & 1 & 1,3 \\
\hline Rue de la Favorite & 33 & $2^{\mathrm{e}}$ moitié $\mathrm{I}^{\mathrm{er}} \mathrm{s}$.-début $\mathrm{II}^{\mathrm{e}} \mathrm{s}$. & 1 & 1 \\
\hline Rue de la Favorite & 233 & $2^{\mathrm{e}}$ moitié $\mathrm{ler}^{\mathrm{er}} \mathrm{s}$ - -début $\mathrm{Il}^{\mathrm{e}} \mathrm{s}$. & 1 & 1,5 \\
\hline Rue de la Favorite & 295 & $2^{\mathrm{e}}$ moitié $\mathrm{I}^{\mathrm{er}} \mathrm{s}$.-début II $\mathrm{s}$. & 1 & 1,4 \\
\hline Rue de la Favorite & 8 & $2^{\mathrm{e}}$ moitié $\mathrm{I}^{\mathrm{er}} \mathrm{s}$.-début $\mathrm{II}^{\mathrm{e}} \mathrm{s}$. & 1 & 1,18 \\
\hline Rue de la Favorite & 132 & $2^{\mathrm{e}}$ moitié $\mathrm{l}^{\mathrm{er}} \mathrm{s}$.-début $\|^{\mathrm{e}} \mathrm{s}$. & 1 & 1,35 \\
\hline Rue de la Favorite & 52 & $2^{\mathrm{e}}$ moitié $\mathrm{I}^{\mathrm{er}} \mathrm{s}$.-début $\mathrm{Il}^{\mathrm{e}} \mathrm{s}$. & 4 & 1,6 \\
\hline Rue de la Favorite & 59 & $1^{\text {re }}$ moitié $\|^{\mathrm{e}} \mathrm{s}$. & 1 & $?$ \\
\hline Rue de la Favorite & 160 & $1^{\text {re }}$ moitié $\|^{\mathrm{e}} \mathrm{s}$. & 1 & 1,2 \\
\hline Rue de la Favorite & 231 & $1^{\mathrm{re}}$ moitié $\|^{\mathrm{e}} \mathrm{s}$. & 1 & 1,2 \\
\hline Rue de la Favorite & 29 & $1^{\text {re }}$ moitié $I^{\mathrm{e}} \mathrm{s}$. & 1 & 1,2 \\
\hline Rue de la Favorite & 27 & $1^{\text {re }}$ moitié $I^{\mathrm{e}} \mathrm{s}$. & 1 & 1,4 \\
\hline Rue de la Favorite & 138 & $1^{\text {re }}$ moitié $I^{\mathrm{e}} \mathrm{s}$. & 1 & 1,5 \\
\hline Rue de la Favorite & 53 & $1^{\text {re }}$ moitié $\|^{\mathrm{e}} \mathrm{s}$. & 1 & 1,45 \\
\hline Rue de la Favorite & 201 & $1^{\text {re }}$ moitié $I^{\mathrm{e}} \mathrm{s}$. & 2 à 4 & 1,6 \\
\hline Rue de la Favorite & 24 & $1^{\text {re }}$ moitié $\mathrm{Il}^{\mathrm{e}} \mathrm{s}$. & 3 & 1,4 \\
\hline Rue du Docteur-Horand & 93 & fin $I^{\text {er }}$ S.-début II $\mathrm{s}$. & \begin{tabular}{|c|} 
non \\
étudié
\end{tabular} & $1,50 \times 0,75$ \\
\hline Voie de l'Océan & 251 & 3 premiers quarts $\|^{\mathrm{e}} \mathrm{s}$. & 1 & $1,40 \times 0,50$ \\
\hline Voie de l'Océan & 235 & 3 premiers quarts $\mathrm{Il}^{\mathrm{e}} \mathrm{s}$. & 1 & $0,80 \times 0,37$ \\
\hline Voie de l'Océan & 152 & $3^{\mathrm{e}}$ quart II -début III $\mathrm{s}$. & 1 & $1,20 \times 0,60$ \\
\hline Voie de l'Océan & 153 & $1^{\text {re }}$ moitié $I^{\text {er }} \mathrm{s}$. & \begin{tabular}{|c|}
2 \\
(1 adulte \\
1 enfant) \\
\end{tabular} & $0,50 \times 0,30$ \\
\hline Rue du Chapeau-Rouge & 166 & début $\|^{\mathrm{e}} \mathrm{s}$. & 1 & $1,40 \times 0,65$ \\
\hline Quai Arloing & 33 & fin Ier s.-début II s. & 1 & $1,60 \times 0,70$ \\
\hline Quai Arloing & 87 & fin Ier s.-début II s. & 1 & $1,40 \times 0,56$ \\
\hline 41 , rue Joliot-Curie & - & $2^{\mathrm{e}}$ moitié $\mathrm{l}^{\mathrm{er}} \mathrm{s}$. & 1 & $2,30 \times 0,90$ \\
\hline 62 , rue du Commandant-Charcot & 2 & $2^{\mathrm{e}}$ moitié $\mathrm{I}^{\mathrm{er}} \mathrm{s}$. & $\begin{array}{c}1 \text { sujet } \\
(1-5 \text { ans })\end{array}$ & $\geq 1,30 \times 0,55$ \\
\hline
\end{tabular}

Tabl. VIII - Longueur des bûchers en fosse à Lyon (sélection de sites) : NMI, nombre minimum d'individus. 


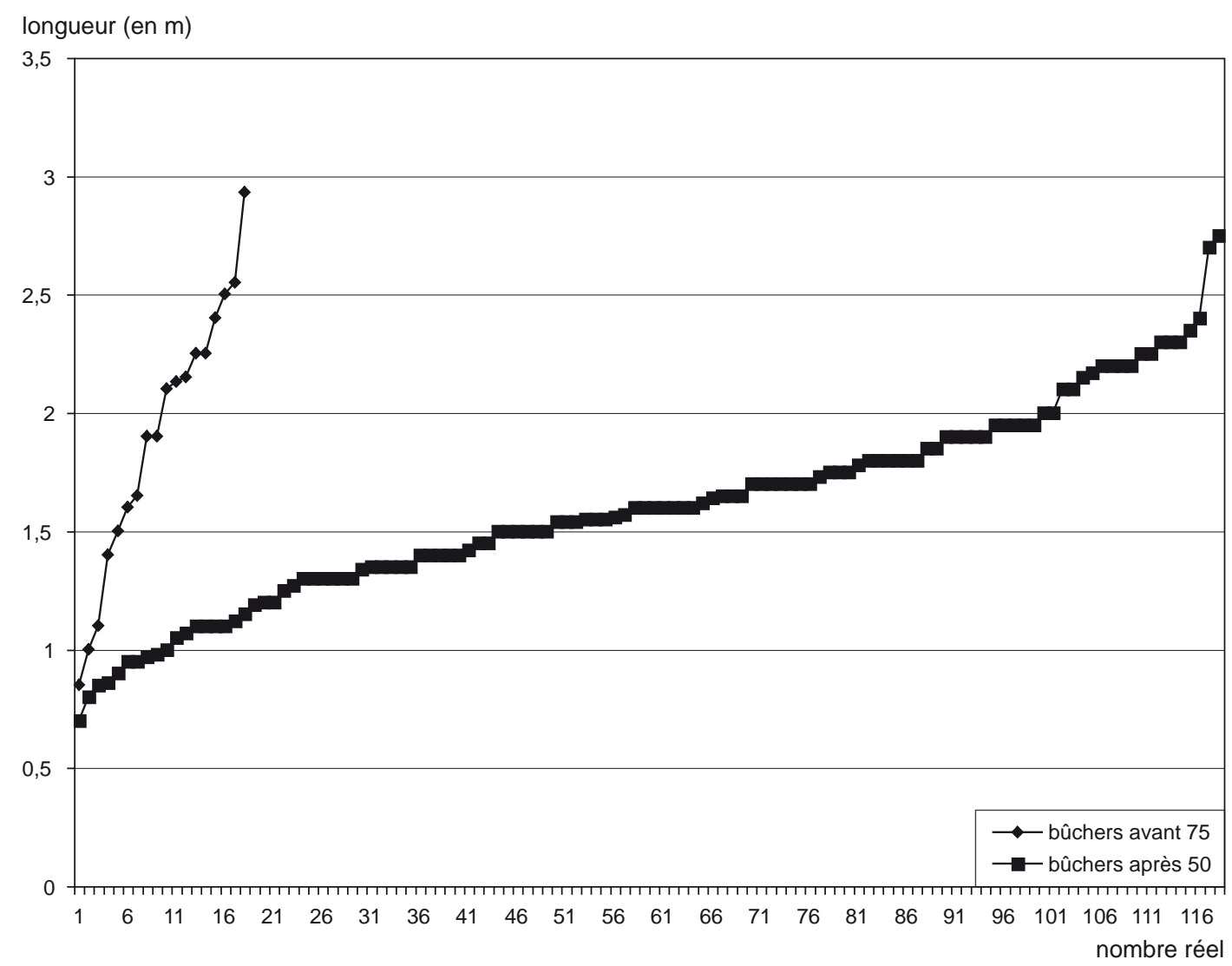

Fig. 75 - Évolution de la longueur des bûchers dans les ensembles de Vernègues (les Communaux de Saint-Cézaire), Saint-Paul-Trois-Châteaux (le Valladas), Aix-en-Provence (ZAC Sextius-Mirabeau) et Marseille (Sainte-Barbe) (DAO : V. Bel, Inrap).

1,35 m (min. 0,66 m, max. 1,35 m, écart-type de 0,23) pour les cinquante années qui suivent et à 1,45 m (min. 1,45 m, $\max .2,05 \mathrm{~m}$, écart-type de 0,24) pour le $\mathrm{II}^{\mathrm{e}} \mathrm{s}$. (Tranoy, 1995b, tabl. 20, p. 695-696). Il y a donc, sur ce site, une réelle diminution de la taille des bûchers entre la première moitié du I ${ }^{\mathrm{er}} \mathrm{s}$. et la période comprenant la seconde moitié $\mathrm{du} \mathrm{I}{ }^{\mathrm{er}} \mathrm{s}$. et le $\mathrm{II}^{\mathrm{e}} \mathrm{s}$. $(\mathrm{t}=2,7$ est compris entre 0,01 et 0,001$)$. Or, les bûchers de la Favorite, qui font monter la longueur moyenne de ceux du ${ }^{\mathrm{er}} \mathrm{s}$. de Lyon à 1,60 m (écart-type de $0,50)$, constituent la quasi-totalité des données antérieures à 50 apr. J.-C. à Lyon (vingt sur vingt-deux). Dès la seconde moitié du $\mathrm{I}^{\mathrm{er}}$ s., la longueur moyenne des bûchers lyonnais descend à 1,40 $\mathrm{m}$ (écart-type de 0,66) et est de 1,30 m (écart-type de 0,34) au $\mathrm{II}^{\mathrm{e}} \mathrm{s}$. Il en ressort que les bûchers les plus grands sont effectivement les plus anciens, mais que, quasiment fondé sur un seul site, ce constat demanderait à être validé par l'apport de données nouvelles. Dans le Sud, il semble que cette évolution soit marquée par une plus grande amplitude des valeurs, alors qu'au départ on a seulement des fosses moyennes ou grandes.
Les creusements de taille modeste n'ont pas forcément été réservés aux crémations individuelles et uniques. Par exemple, les bûchers 24 et 52 de la Favorite, qui livrent les restes de trois et quatre sujets, ont une longueur très proche de la moyenne de chacune des phases auxquelles ils appartiennent : 1,40 m pour le bûcher 24 et 1,60 m pour le bûcher 52 (moyenne de 1,35 m et 1,45 m). À l'inverse, il existe de très nombreux cas de bûchers individuels de grandes dimensions. On peut citer l'exemple de la fosse $71 \mathrm{du}$ Valladas à Saint-Paul-Trois-Châteaux qui mesurait $1,90 \mathrm{~m}$ de longueur et 1,40 $\mathrm{m}$ de largeur et dont divers éléments de mobilier brûlé ont pu être reliés à ceux d'une structure de dépôt implantée au sein du même enclos funéraire (tombe 70). C'est aussi le cas des grandes fosses de Peyre Plantade à Clermont-l'Hérault (Hérault) (2,93 m de longueur et 2,57 $\mathrm{m}$ de largeur), de Pont de Pierre à Bollène, dans le Vaucluse, (2,50 m x 1,50 m) (Alfonso, 2002), du Pôle Synéo à Chartres, en Eure-et-Loir (4,34 m x 2,58 m) ou de Saint-Fréjus à Marennes, dans le Rhône (fosses de 2,55 m et 2,10 m de longueur), et de celles de nombreux sites lyonnais, 


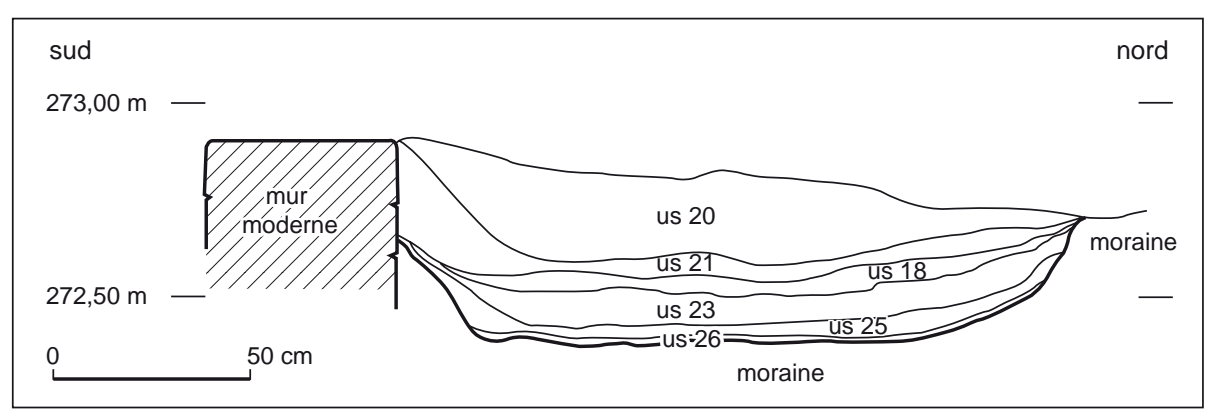

Fig. 76 - Stratigraphie d'un bûcher en fosse : bûcher 1000 du 62 avenue du Point-du-Jour à Lyon (relevé : L. Kuntz, Inrap; DAO : G. Macabéo, Inrap).

qui renfermaient les restes d'un seul sujet. Dans cette série des grands bûchers en fosse, l'exemplaire augustéen de Villelongue-d'Aude (fouille M. Passelac, inédite) ou celui du col de Ceyssat à Saint-Genès-Champanelle, dans le Puy-de-Dôme (milieu du II ${ }^{\mathrm{e}}$ s.) (Trément, Humbert, 2005) constituent des hapax par leurs dimensions tout à fait exceptionnelles $\left(20 \mathrm{~m}^{2}\right.$ et $\left.18,24 \mathrm{~m}^{2}\right)$.

Ces différences de dimensions ne peuvent guère être interprétées. Sont-elles liées à la part d'ostentation que la famille entend exprimer lors des funérailles ? Ou bien traduisent-elles des types crématoires un peu différents ? Les recherches menées en Asie par G. Grévin ont mis en évidence une assez grande diversité dans la manière de brûler les corps (Grévin, 2005). Sur le continent indien, les bûchers sont par exemple souvent de petite taille, ce qui fait que le corps dépasse aux deux extrémités ; en cours de crémation, l'officiant rabat alors les membres au centre (Grévin, communication personnelle). Selon que l'on retienne l'une ou l'autre des explications, la généralisation des petits bûchers en fosse dès le milieu du $\mathrm{I}^{\mathrm{er}} \mathrm{s}$. traduirait dans le premier cas l'accès à ce mode crématoire à un plus grand nombre, et dans le second, une diversification des types de crémation.

Quelle que soit leur taille, les bûchers en fosse conservent presque toujours une couche de résidus charbonneux plus ou moins épaisse tapissant le fond de la fosse. Souvent cette couche comporte des fragments de bois calciné de bonne taille, parfois les bûches entières, comme dans certains bûchers de la Favorite ou du Sextant rue du CommandantCharcot à Lyon. En l'absence de trace de rubéfaction, la présence de nombreuses bûches calcinées associées à des ossements et du mobilier brûlé constitue un indice suffisant pour identifier une structure de crémation, parce que celles-ci sont généralement absentes dans les structures de dépôt. Toutefois, lorsque le morceau de bois est isolé dans une couche peu charbonneuse, comme c'est le cas dans la fosse 4 de l'ensemble funéraire du Pont de Pierre (Alfonso, 2002, p. 798), l'hypothèse d'un dépôt secondaire peut être envisagée et il n'est pas toujours possible de trancher. La couche de résidus apparaît souvent plus épaisse le long des parois qu'au centre et elle est dans bien des cas caractérisée par une organisation interne nettement stratifiée, avec, souvent, une localisation des restes de bois carbonisé les plus importants dans la partie inférieure. La partie supérieure, moins charbonneuse, concentre la plupart des ossements et du mobilier brûlés, ainsi que des inclusions de terre rubéfiées provenant de l'effritement des parois de la fosse.

La stratification type du bûcher peut être illustrée par celui fouillé au 62 avenue du Point-du-Jour à Lyon (fig. 76) : le fond est tapissé d'une fine couche de sédiment remanié par le creusement de la fosse (us 26), qui est recouverte d'une couche cendreuse mêlée à quelques charbons et artefacts brûlés (us 25). Au-dessus se trouve une couche grasse très noire avec de gros charbons, très riche en os et en mobilier calcinés (us 23), que surmontent deux couches de même nature mais plus hétérogènes (us 18 et 21), avec notamment des matériaux provenant de l'encaissant plus nombreux dans la dernière (us 21). Celle-ci possède, comme c'est souvent le cas, un profil concave et est surmontée d'un remblai pauvre ou dépourvu de résidus (us 20).

Ces indices ne sont pas toujours tous réunis et, pris isolément, ne constituent pas des arguments déterminants, surtout lorsque la stratigraphie n'est pas aussi différenciée. Aussi, est-il nécessaire de tenter de vérifier l'hypothèse d'une crémation in situ en ayant recours à l'analyse de la répartition spatiale des restes humains et des vestiges mobiliers, en supposant que la couche de crémation n'a pas été remaniée (voir chapitre III). Cette analyse, qui implique un important travail de tri et de détermination, nécessite des compétences en ostéologie humaine. Un des indices les plus évidents d'une crémation sur place est la conservation de connexions anatomiques, à condition cependant que 
cette observation soit répétée et surtout qu'elle soit croisée avec une répartition cohérente de l'ensemble des régions anatomiques. L'analyse spatiale des bûchers passe donc par un enregistrement exhaustif des pièces osseuses (même les plus petites) sur la surface du bûcher. En pratique, cela ne peut être réellement réalisé que par un prélèvement en masse d'unités d'égales dimensions plus ou moins grandes selon les besoins (Bel, 1996).

Il arrive que dans leur ensemble, les éléments principaux : rubéfaction en place, répartition cohérente des vestiges anatomiques et stratigraphie, ne soient pas suffisamment explicites ; dans ce cas, il n'est pas possible d'interpréter la structure (voir chapitres III et IV).

\section{LA DIFFUSION ET LA CHRONOLOGIE DES BÛCHERS EN FOSSE}

Le recensement dans la documentation disponible des bûchers en fosse avérés, soit par des marques d'altération par le feu, soit par la répartition des restes osseux humains, révèle une répartition très hétérogène tant géographique que chronologique de ce mode de crémation (tabl. IX et X). On le rencontre en effet principalement à Lyon et dans les ensembles funéraires périurbains de Narbonnaise récemment étudiés. Si, en nombre de structures, le Languedoc et l'Auvergne sont nettement moins pourvus que la vallée du Rhône et la Provence, la multiplication des découvertes au cours des dix dernières années grâce au développement de l'archéologie préventive montre qu'en proportion, ce mode de crémation est également bien implanté à l'ouest du Rhône. Il semble que la répartition des bûchers en fosse est pour une bonne part liée aux aléas de la recherche archéologique et il serait réducteur d'en faire un phénomène essentiellement urbain comme nous le pensions auparavant (Bel, Tranoy, 1993b, p. 98).

Les séries urbaines du Midi montrent d'ailleurs des situations contrastées, si l'on compare par exemple la part des bûchers en fosse sur le total des structures qui se rapportent à la crémation (tabl. X) dans l'ensemble funéraire de SainteBarbe (Moliner et al., 2003, p. 37) et dans ceux de Fréjus, dans le Var (Béraud, Gébara, 1987a). Si les ensembles funéraires aixois comportent quelques exemplaires de bûchers, la fouille réalisée récemment à Riez (Alpes-de-Haute-Provence) n'a livré aucune structure de ce type (fouille inédite, P. Borgard ; Borgard et al., 2005, p. 40). Alors que sur le site du Valladas, les bûchers représentent près de $20 \%$ des structures funéraires (Bel et al., 2002, p. 85), dans la colonie voisine d'Orange, l'enclos funéraire B du site de Fouches Vieilles utilisé du milieu du $\mathrm{I}^{\mathrm{er}}$ s. au milieu du $\mathrm{III}^{\mathrm{e}}$ s. ne comporte qu'un seul bûcher en fosse et vingt-trois dépôts de crémation (Mignon, 2001b, p. 132). Dans le cas de Vaison-la-Romaine (Vaucluse), les conditions dans lesquelles les découvertes funéraires ont été effectuées, pourraient expliquer l'absence de bûcher en fosse (Provost, Meffre, 2003, p. 309 et p. 316-317).

On n'est pas en mesure de déterminer si les variations observées traduisent des différences régionales ou plus locales, car le caractère souvent hétérogène des sources permet rarement de comparer entre eux différents «quartiers funéraires » d'une même agglomération. À Fréjus, on n'observe pas de différence entre les deux ensembles funéraires qui ont été fouillés. On dénombre seulement un bûcher en fosse pour 94 structures se rapportant à la pratique de la crémation au Pauvadou (Béraud, Gébara, 1987a) et quatre bûchers (deux « ustrina » et deux «busta») pour 204 structures à Saint-Lambert (Béraud, Gébara, 1987a). À Nîmes, les bûchers ont été retrouvés dans plusieurs ensembles funéraires périurbains distincts. Dix d'entre eux (soit la moitié des exemples attestés) proviennent de deux sites proches, fouillés dans le cadre d'opérations préventives en 1996 et 2003 aux n ${ }^{\text {os }} 59$ et 78 de l'avenue Jean-Jaurès, le long des voies issues de la porte du Cadereau, au sud-ouest de la ville (fouilles de M.-L. Hervé et V. Bel). Trois autres ont été fouillés par F. Mazauric en 1911 au n 594 de l'avenue du Maréchal-Juin, le long de la voie Domitienne (Fiches, Veyrac dir., 1996, point 448). Un autre a été découvert en 1890 par A. Vigne, cette fois le long du tronçon oriental de la voie Domitienne issu de la porte d'Auguste ( $\mathrm{n}^{\circ} 53$ de la route de Beaucaire, $c f$. Fiches, Veyrac dir., 1996, p. 404). En revanche, les différentes interventions qui ont été menées sur des espaces funéraires dans ce même secteur (route de Beaucaire), n'en n'ont pas livré (Manniez, 1995 ; Hervé, 1996, p. 82-83 ; Bel, 1999a, p. 69-70).

À Lyon, la situation est toute aussi contrastée. Sur les grands sites, les proportions varient énormément, puisque les bûchers en fosse peuvent représenter une part infime des structures (au moins 3 sur 86 dépôts secondaires, voie de l'Océan ; 1 sur 25 rue du Chapeau-Rouge ; 1 sur 8 au quai Arloing ; 1 sur 54 aux 77-79 rue Pierre-Audry à la fin du $\mathrm{II}^{\mathrm{e}}$ s. et au $\mathrm{III}^{\mathrm{e}}$ s.), ou une part importante à très importante (7 bûchers pour 6 dépôts secondaires pour l'époque claudienne rue des Granges ; 6 pour 1 au 54 rue PierreAudry ; 131 pour 112 à la Favorite). D'autres sites, comme Saint-Irénée ( $\mathrm{II}^{\mathrm{e}}-\mathrm{III}^{\mathrm{e}} \mathrm{s}$.), les rues Rochette et Chevrier $\left(\mathrm{III}^{\mathrm{e}} \mathrm{s}\right.$.) n'en ont livré aucun, tandis que des bûchers constituent la totalité ou la quasi-totalité de l'occupation funéraire au 77-79 rue Pierre-Audry au I ${ }^{\mathrm{er}}$ s. par exemple. Quelques-uns, 







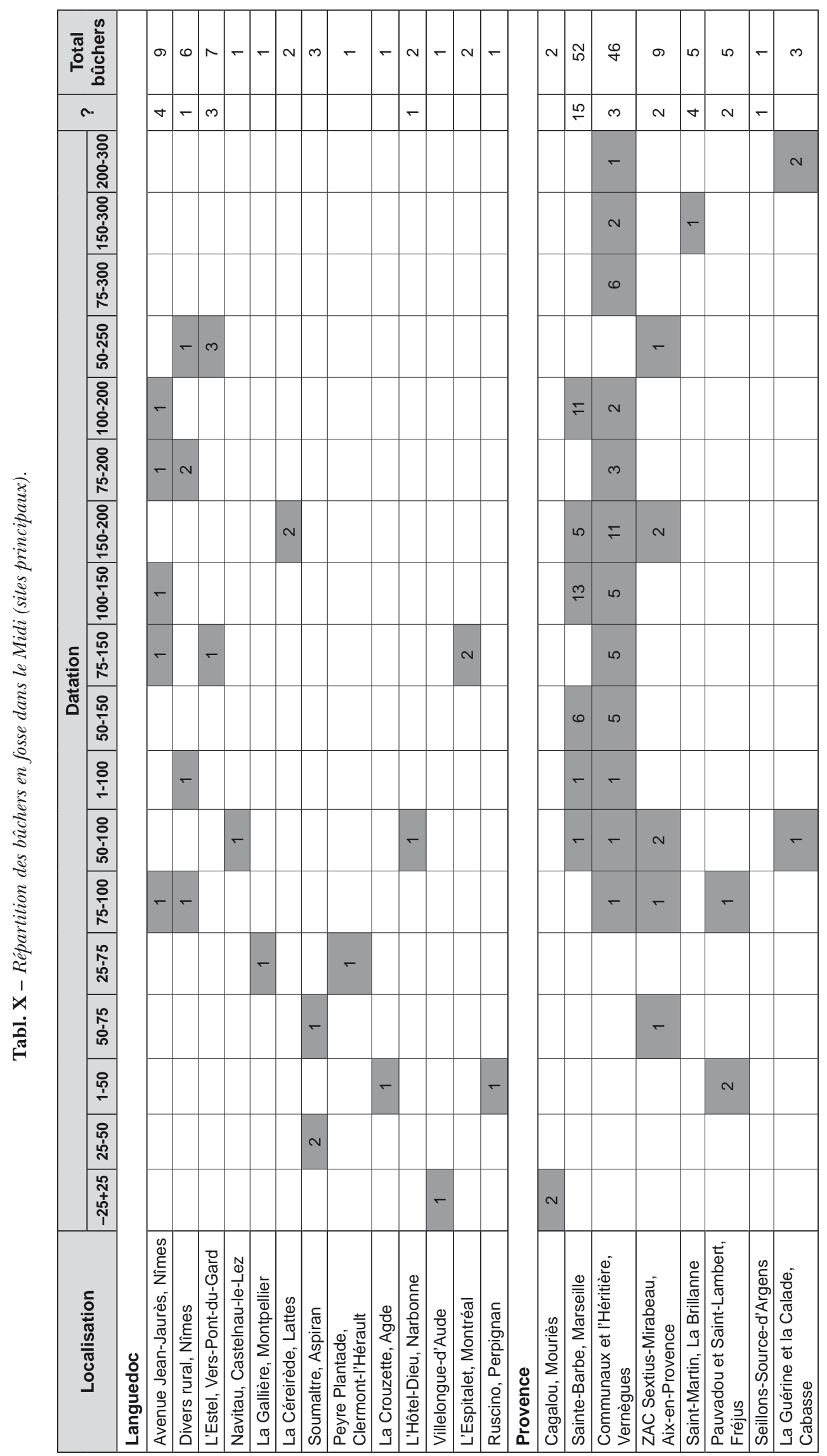


accompagnés ou non de structures de dépôts, ont été exhumés lors d'interventions ponctuelles réalisées sur de petites surfaces (rue du Docteur-Horand, 62 avenue du Point-du-Jour). Toutefois, il y a fort à parier que ces proportions sont en partie fonction de la surface explorée, ces grands bûchers étant le plus souvent réunis dans un secteur défini, comme le montrent les sites de la Favorite, du 41 rue Joliot-Curie, du Sextant rue du Commandant-Charcot, etc. En l'état des connaissances sur Lyon, il semblerait que le bûcher en fosse est plus fréquent dans l'actuel $5^{\mathrm{e}}$ arrondissement (secteur de Fourvière, à proximité de la ville haute antique) que dans le $9^{\mathrm{e}}$ (secteur de Vaise à distance de la ville), bien que le site de Villa Montel, jamais étudié, en livre manifestement un certain nombre. Les deux autres grands sites périurbains fouillés en Rhône-Alpes, le Replat à Aime (Savoie) et le Valladas comptent respectivement 6 et 39 bûchers pour 40 et 180 structures secondaires. L'inventaire réalisé par C. Mondanel ne donne aucun bûcher de ce type pour les contextes périurbains de Clermont-Ferrand, ni même pour Lezoux (Mondanel, 1982). Les quelques exemples connus proviennent de contextes ruraux.

Les bûchers en fosse ne se rencontrent pas uniquement dans les ensembles funéraires de chefs-lieux de cité. Ils sont également associés aux agglomérations secondaires, comme à Vernègues, sur les sites de l'Héritière et des Communaux à Saint-Cézaire qui sont composés presque exclusivement de ce type de structures (Chapon et al., 2004). Ils apparaissent également près des agglomérations de Cabasse dans le Var (Bérard, 1961 et 1980), de Castelnau-le-Lez (Ramonat, Sahuc, 1988), de Clermont-l'Hérault. Des bûchers sont également mentionnés, sans précision, dans l'ensemble funéraire de Lattes, riche de 170 structures liées à la crémation, malheureusement non publiées, à l'exception de la verrerie et des inscriptions (Demougeot, 1972 ; Pistolet, 1981 ; Vial, 2003, p. 212).

Aujourd'hui, la documentation disponible ne montre pas de ce point de vue d'opposition entre espace urbain et espace rural contrairement à ce qui avait pu être constaté en 1993 (Bel, Tranoy, 1993b ; Bel et al., 2002, fig. 613). Le développement des décapages archéologiques en contexte rural a permis de multiplier les découvertes de tombes dispersées et de renouveler nos connaissances sur les pratiques funéraires dans les campagnes. En Languedoc par exemple, on observe que la plupart des ensembles funéraires du Haut-Empire récemment fouillés, comme l'Estel à VersPont-du-Gard (Vacca-Goutoulli, 2001), Soumaltre à Aspiran (Thernot et al., 2004), la Céreirède à Lattes (Bel, Conche, 2003), Gallière à Montpellier ont livré au moins un bûcher

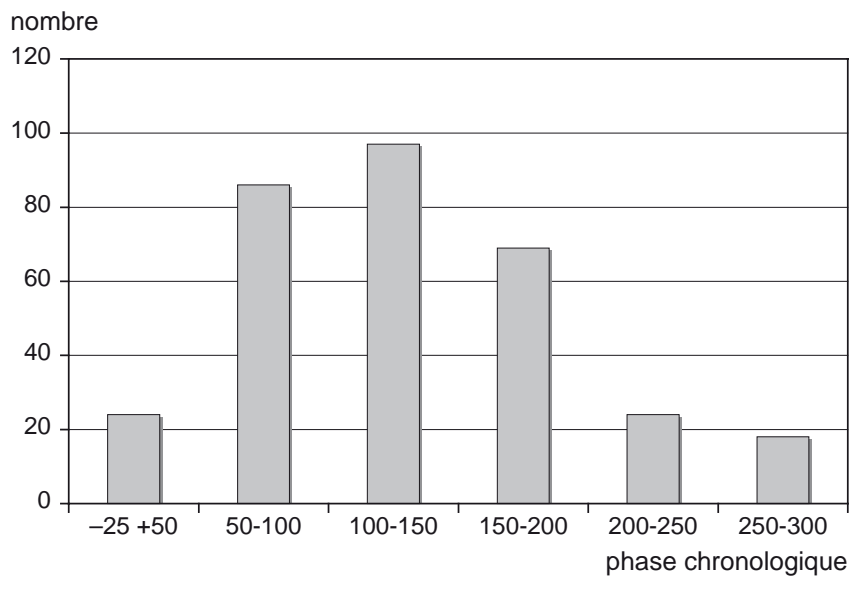

Fig. 77 - Diagramme cumulatif par période chronologique des effectifs de bûchers recensés dans le midi de la Gaule : moyenne vallée du Rhône, Provence, Languedoc (DAO : V. Bel, Inrap).

en fosse. À Nîmes, c'est le cas pour quatre (et peut-être cinq) sites funéraires sur sept dans la proche campagne : Mas de Vignoles IV, Mas de Vignoles IX, Mas Carbonnel, Magaille. Les nombreux exemples relevés dans les campagnes des régions Rhône-Alpes et Auvergne, montrent que, pour la plupart, les bûchers en fosse sont associés à des domaines, comme à Saint-Fréjus, Champ Chalatras aux Martresd'Artière, probablement à la Grande Borne (ClermontFerrand) et aux Quaires (Les Martres-d'Artière), à La Boisse dans l'Ain (fouille inédite de P. Nuoffer, Archéodunum), aux Feuillis ou aux Luèpes à Saint-Priest (Rhône) ou encore à la Brunerie à Voiron (Isère). Ces bûchers peuvent être isolés ou regroupés en lots de moins de cinq unités, et se situer ou non parmi des structures de dépôt.

La répartition des bûchers par période paraît plus significative. Les structures de ce type sont en effet assez rares avant le milieu, voire le troisième quart, du $\mathrm{I}^{\mathrm{er}} \mathrm{s}$. apr. J.-C. (fig. 77). Les plus anciens sont localisés principalement à Lyon et en Languedoc occidental. Pour le Languedoc, citons Villelongue-d'Aude, Ruscino à Perpignan, dans les Pyrénées-Orientales (Marichal, 1998, p. 125), Aspiran, Soumaltre (Thernot et al., 2004) et Clermont-l'Hérault, Peyre Plantade dans la vallée de l'Hérault. Des exemplaires d'époque augustéenne ont également été mis au jour à SaintLambert, à Fréjus (Béraud, Gébara, 1987a et b) et à Mouriès dans les Alpilles (Bouches-du-Rhône, site du Cagalou, $c f$. Marcadal, Paillet., 2003, p. 270-274). À Lyon, les bûchers en fosse datés de la période augustéenne proviennent des sites de la rue du Commandant-Charcot (les Roses) et de la rue des Granges, mais des exemplaires de la première moitié $\mathrm{du} \mathrm{I}^{\mathrm{er}} \mathrm{s}$. de notre ère sont également relevés à la Favorite. 
En dehors de Lyon, on en trouve pour la même période en milieu périurbain au Valladas dans la Drôme et à Annecy en Haute-Savoie. En contexte rural, les faits sont moins clairs : les sites de Chalépont à Montalieu-Vercieu, en Isère (De Klijn, 1987) et de Corbas, dans le Rhône, livrent des structures du début du $\mathrm{I}^{\mathrm{er}} \mathrm{s}$. interprétées comme telles par les fouilleurs, mais les arguments nous paraissent faibles.

Leur grande période est assurément la seconde moitié $\mathrm{du} \mathrm{I}{ }^{\mathrm{er}} \mathrm{s}$. et la première moitié $d u \mathrm{II}^{\mathrm{e}} \mathrm{s}$., avec une envolée spectaculaire entre 50 et 100. Le site de la Favorite est à cet égard bien représentatif : $59 \%$ des bûchers en fosses datés se situent dans la seconde moitié du $\mathrm{I}^{\mathrm{er}} \mathrm{s}$. tandis que les périodes comprises entre 0 et 50 et entre 100 et 150 comptent pour $19 \%$ et $18 \%$. Dès 150, l'utilisation du bûcher en fosse chute brutalement à Lyon (3 sur les 14 fouillés aux 77-79 rue Pierre-Audry et 4 sur les 110 datés de la Favorite). En revanche, sur les sites urbains situés en dehors de Lyon, les bûchers restent nombreux durant tout le $\mathrm{II}^{\mathrm{e}} \mathrm{s}$. On en trouve également en contexte rural au II $^{\mathrm{e}}$ s., par exemple aux Luèpes à Saint-Priest dans le Rhône ou à la Grande Borne à Clermont-Ferrand, dont certains présentent des caractères ostentatoires que l'on rencontre plus fréquemment au $\mathrm{I}^{\mathrm{er}} \mathrm{s}$. en contexte urbain. Dans le Midi, la pleine période d'utilisation des bûchers en fosse couvre la seconde moitié du I ${ }^{\mathrm{er}} \mathrm{s}$. et tout le $\mathrm{II}^{\mathrm{e}} \mathrm{s}$. apr. J.-C. Au cours de cette phase, les effectifs paraissent stables, du moins globalement : 23 exemplaires au moins sont datés de la seconde moitié du $\mathrm{II}^{\mathrm{e}}$ s., mais la moitié provient du seul site de Vernègues. Par la suite, avec l'abandon rapide de la pratique de la crémation, les occurrences diminuent sensiblement puisqu'on n'attribue plus que trois exemplaires au $\operatorname{III}^{\mathrm{e}} \mathrm{s}$. Les rares données de Midi-Pyrénées ne permettent pas d'apprécier l'évolution du bûcher en fosse. Les trois exemples recensés (la caserne Niel à Toulouse, fouillée en 2004, le Larsou à Réalville dans le Tarn-et-Garonne et le jardin du Séminaire à Rodez) sont postérieurs au début $d u \mathrm{II}^{\mathrm{e}} \mathrm{s}$.

Pour suivre la dynamique et les modalités de cette évolution, il convient de tenir compte de la diversité de formes des bûchers. Ce type de structure recouvre en effet des dispositifs et des modes de fonctionnement variés.

\section{DES BÛCHERS UTILISÉS UNE SEULE FOIS OU À PLUSIEURS REPRISES}

Dans quelques cas, peu nombreux, il existe des indices convaincants d'une réutilisation de la fosse pour des crémations successives. Cette réutilisation semble sans rapport avec la taille du bûcher, sa forme ou son éventuelle construction.
À Lyon, quelques bûchers renferment les restes de plusieurs sujets, mais faute d'avoir pu réaliser un enregistrement in situ adapté des vestiges, il n'a jamais été possible de démontrer si les crémations ont été simultanées ou successives, sachant qu'en théorie, la crémation multiple est attestée dans l'Antiquité au moins par le texte des Saturnales de Macrobe (VII, 7, 5). Il s'agit toujours de bûchers non construits. À la Favorite, sur un échantillon de 42 bûchers, 10 renfermaient deux sujets et 2 en contenaient trois (Tranoy, 1995b, p. 680-681). Dans tous les cas, il n'est décrit qu'une seule couche de résidus. Un bûcher de deux individus est mentionné aux 77-79 rue Pierre-Audry (3148). L'auteur ne distingue qu'un remplissage composé des restes d'un adulte et d'un jeune enfant, ce qui pourrait être le signe d'une crémation simultanée, étant donné que ce type d'assemblage (jeune enfant et sujet plus âgé) est très fréquemment relevé dans les dépôts secondaires (voir infra, p. 219). À la Favorite, le curage d'un bûcher en fosse (bûcher 272) a suggéré à l'auteur l'hypothèse que l'on avait prévu de l'utiliser pour une autre crémation (Tranoy, 1995b, p. 679). Les parois et le fond de cette fosse, d'une taille de $2 \mathrm{~m}$ sur $1,50 \mathrm{~m}$, étaient très indurées sur une épaisseur plus importante que dans les autres bûchers, mais il ne subsistait plus de résidus charbonneux.

Le bûcher 1004 de l'ensemble funéraire du 78 avenue Jean-Jaurès à Nîmes fournit un exemple clair de réutilisation. Il s'agit d'une fosse quadrangulaire aux parois verticales et au fond plat, d'environ $1,95 \mathrm{~m}$ de longueur, de 1,50 $\mathrm{m}$ de largeur et de $0,54 \mathrm{~m}$ de profondeur. Les parois étaient en partie rubéfiées de même que le sol de circulation au contact de la fosse (fig. 78, surface us 1208). Le fond est recouvert par une couche de limon argileux très rubéfié (fig. 78, us 1279), avec quelques charbons de bois, de rares fragments de céramique et d'os brûlés de très petite taille. Ces vestiges témoignent d'une première utilisation de la structure pour la crémation, suivie d'un curage presque complet. Quatre grandes dalles de calcaire ont ensuite été installées sur ce niveau, contre les parois de la fosse, de manière à constituer un coffrage (fig. 79). Deux dalles présentaient sur leurs faces internes, très abîmées et délitées, des traces nettes de rubéfaction qui résultent d'une exposition au feu prolongée ou répétée. Le comblement du coffrage comportait, à la base et le long des parois, un amas de résidus très charbonneux de $0,18 \mathrm{~m}$ d'épaisseur, scellé par des remblais de terre et de blocs qui constituent la couche de condamnation de la structure. La couche de crémation a livré des fragments osseux humains brûlés d'au moins un sujet, de rares fragments de céramique et de verre, brûlés 


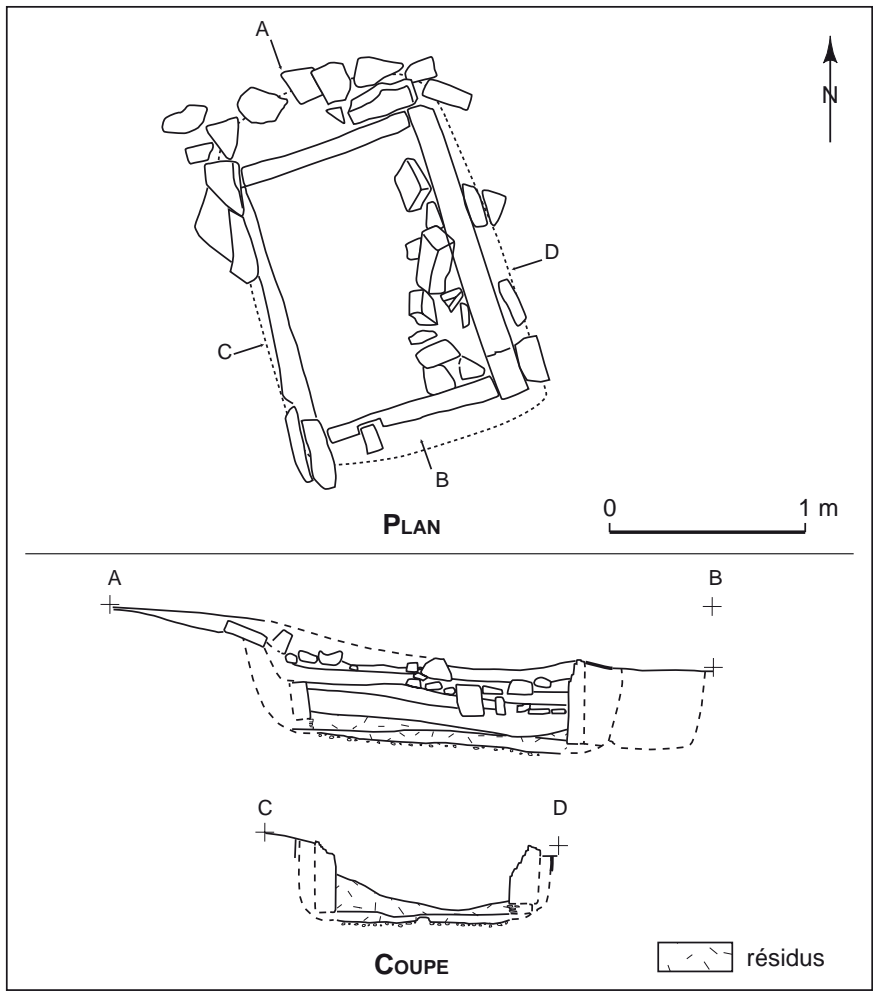

Fig. 78 - Exemple de bûcher en coffrage de dalles : fosse 1004 du 78 avenue Jean-Jaurès à Nîmes, Gard (relevé : V. Bel, Inrap ; DAO : G. Macabéo, Inrap).

ou non, et de nombreux éclats calcaires lamellaires qui pourraient provenir de l'effritement des dalles sous l'action de la chaleur. Une sorte de pavement constitué d'un assemblage de moellons et de blocs de calcaire a été aménagé sur le sol autour de la fosse. Il présentait en plusieurs points des traces de rubéfaction. Sa construction pourrait avoir eu pour fonction de stabiliser les abords de la fosse fragilisés par les crémations successives. Il pourrait s'agir aussi d'un radier destiné à supporter le bûcher funéraire. La construction d'un coffrage, l'aménagement d'un pavement autour de la fosse et la détérioration importante des dalles de calcaire sous l'action du feu pourraient être des indices d'usages multiples. Le fonctionnement de la structure est daté de la fin du ${ }^{\mathrm{er}}$ s. ou du $\mathrm{II}^{\mathrm{e}}$ s. apr. J.-C.

\section{BÛCHERS EN FOSSE AUX PAROIS OU AU FOND CONSTRUITS}

Outre l'exemple cité ci-dessus du 78 avenue Jean-Jaurès à Nîmes, la documentation disponible pour le Midi fournit d'autres cas de bûchers aux parois ou au fond construits. Des indices permettent parfois de penser que ces structures

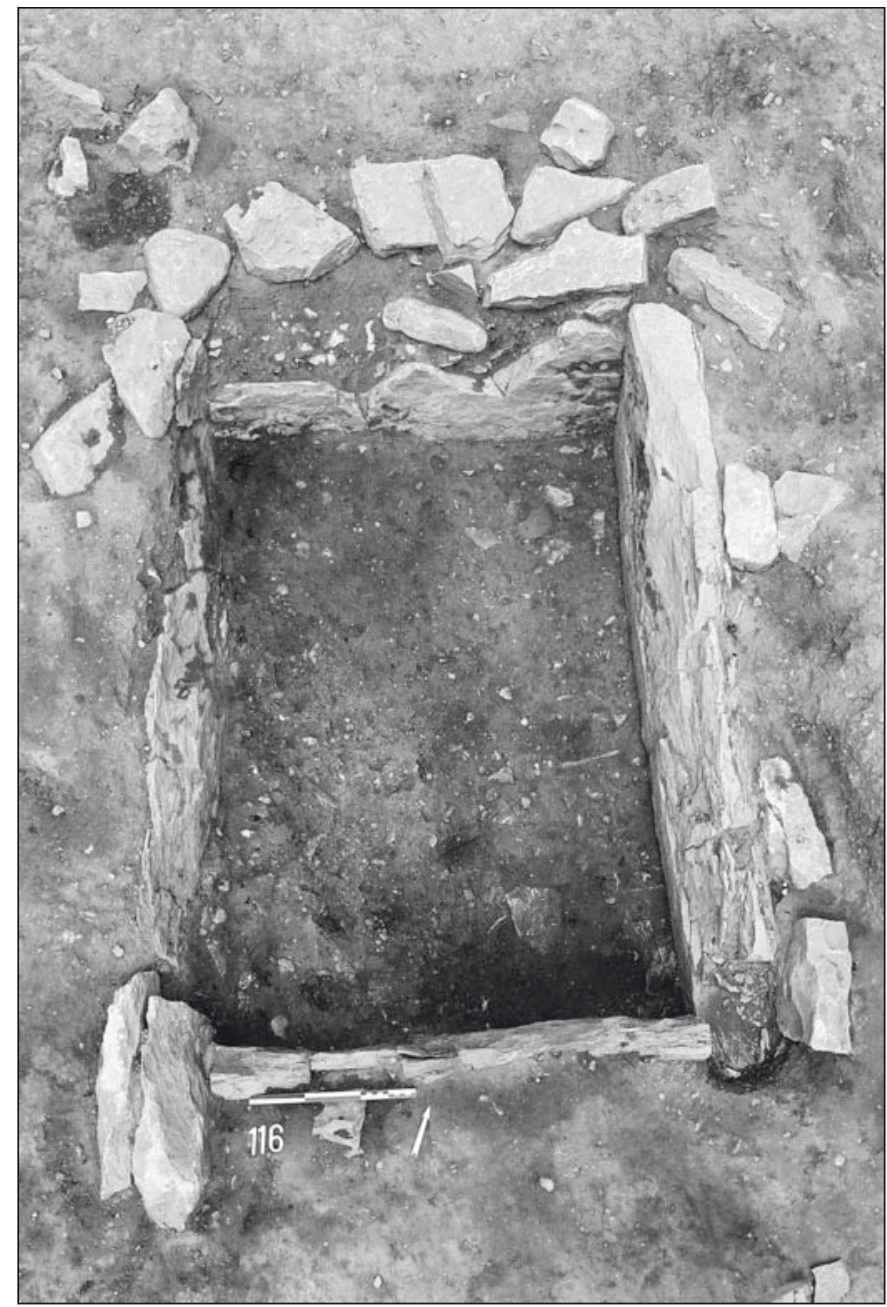

Fig. 79 - Vue du coffrage de dalles du bûcher 1004 du 78 avenue Jean-Jaurès à Nîmes, Gard (cliché : Y. Manniez, Inrap).

ont été conçues et utilisées pour des crémations successives, mais on en possède rarement la preuve.

À Fréjus, sur l'ensemble funéraire de Saint-Lambert, est signalé un bûcher installé dans une grande fosse rectangulaire bordée d'un mur en pierres sèches et pourvue d'une sorte de couloir d'accès (Béraud, Gébara, 1987a, p. 27 et 1987b, p. 186).

À Nîmes, quatre fosses aux parois construites ont été découvertes en 1911, au 594, avenue du Maréchal-Juin (route de Montpellier) (Fiches, Veyrac dir., 1996, p. 448). F. Mazauric les désigne sous les termes d'« ustrina» ou de «bûchers » et mentionne pour deux d'entre elles des traces de rubéfaction et de cendres. Celles-ci étaient parementées avec des maçonneries constituées de fragments de tuiles, de briques et de moellons. Dans un cas, le fond possède un pavement de «dalles de terre cuite » sur un lit de mortier. 


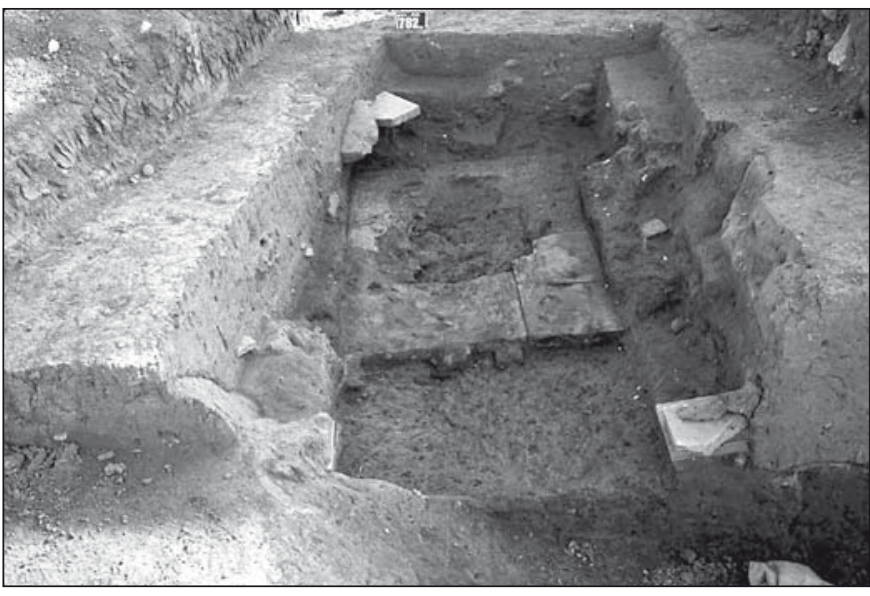

Fig. 80 - Le bûcher construit du Mas de Vignoles IV à Nîmes, Gard (cliché : L. Vidal, Inrap).

Leurs dimensions sont comparables à celles de la fosse $1004 \mathrm{du} 78$ avenue Jean-Jaurès (1,60 m x 0,80 m ; 1,80 m x $0,60 \mathrm{~m})$. Plus récemment, un exemple de bûcher en fosse construit (fig. 80), daté du courant de la première moitié du $\mathrm{II}^{\mathrm{e}}$ s., a été mis au jour sur le site rural du Mas de Vignole IV, à proximité de tombes du $\mathrm{I}^{\mathrm{er}}$ s. av. J.-C. (structure 1638). De dimensions supérieures $(2,40 \mathrm{~m}$ x $1,30 \mathrm{~m})$, il possède un fond recouvert par une couche de mortier de tuileau peigné, incluant des demi-dalles de suspensura (bipedales) et ses parois ont été doublées par un mur constitué d'un parement de dalles carrées de pilettes d'hypocauste, superposées et liées par de la terre (deux assises sont conservées). Ces aménagements, partiellement conservés, semblent avoir fait l'objet d'une destruction volontaire. Des éléments provenant de la destruction sont mêlés au comblement de la fosse constitué d'un limon charbonneux avec des nodules de terre rubéfiée, quelques ossements brûlés (non étudiés) et des fragments de mobilier dont une partie est brûlée. Partout où il est conservé, le sol construit est noirci et sa partie centrale est érodée par l'action de la chaleur.

Le fond des bûchers est rarement aménagé, mais dans l'un des exemplaires de Sainte-Barbe, un lit de pierres tapissait le «fond du creusement » (Moliner et al., 2003, p. 353, tombe 387).

L'ensemble funéraire du Valladas (Bel et al., 2002, p. 356) a livré une fosse aux parois maçonnées dont le parement présentait des traces d'une exposition au feu (comme le fond, non aménagé). De dimensions modestes (longueur: 1,10 m), elle conserve sur le fond (lui aussi altéré) des bûches carbonisées et une couche charbonneuse associée à des restes de vases brûlés et à quelques rares fragments osseux humains brûlés. Les résidus de la crémation ont été scellés par une couche d'argile verte avec des blocs provenant de la destruction de la partie supérieure des parois.

Les bûchers maçonnés en fosse sont également connus en Italie, à Aquilée (Brusin, 1941, p. 209, 211, 216) ou Ostie (Squarciapino dir., 1958, p. 111), en Espagne (Bonsor, 1931), mais aussi en Gaule septentrionale (Fourteau-Bardaji et al., 1993 ; Fayes et al., 1993, p. 90 ; Polfer, 1996, p. 20). Ils sont en revanche totalement absents à Lyon et à Clermont-Ferrand.

\section{LE DISPOSITIF GÉNÉRAL DU BÛCHER}

\section{L'INSTALLATION DU BÛGHER}

\section{BÛCHER CONSTRUIT DANS LA FOSSE}

Les variations de taille et de construction pourraientelles correspondre à des modes de fonctionnement et de crémation différents ? Dans le cas des très grandes fosses, on pourrait imaginer en effet que le bûcher a pu être édifié dans la fosse elle-même, le volume étant suffisant pour permettre une bonne aération de la structure. Des indices relevés lors de la fouille du bûcher 3060 de Peyre Plantade vont dans ce sens. Il s'agit d'une grande fosse quadrangulaire de 2,93 m sur 2,57 m et 0,19 m de profondeur (fig. 81), fortement écrêtée par les labours qui ont fait disparaître à cet endroit le paléosol antique.

Les parois et le fond portent de nombreuses traces d'une exposition au feu dont la répartition, loin d'être uniforme, témoigne de l'implantation du bûcher funéraire dans la fosse elle-même. La rubéfaction concerne en effet une partie des parois sur $0,05 \mathrm{~m}$ à $0,08 \mathrm{~m}$ de haut, la zone inférieure (sur $0,05 \mathrm{~m}$ à $0,10 \mathrm{~m}$ de haut) n'étant pas rubéfiée. Sur le fond, les marques d'oxydation s'organisent en six bandes parallèles entre elles et avec les bords est et ouest de la fosse. Elles sont très irrégulières, de largeurs variables (de 0,06 m à 0,35 m environ), de longueurs inégales, et présentent de nombreuses lacunes. Elles sont toutefois assez régulièrement espacées ce qui tend à montrer qu'il ne s'agit pas d'une disposition fortuite. On est tenté de les interpréter comme les empreintes en négatif des poutres de base du bûcher, ces dernières devant être restituées à l'emplacement des bandes non oxydées. Cette rubéfaction n'a pu se produire qu'en atmosphère oxydante, sur un sol nu ou seulement recouvert d'une faible épaisseur de braises. Selon toute vraisemblance, elle a dû intervenir dans les premières minutes de la crémation, au moment de l'embrasement du bûcher, avant que la construction ne s'effondre et que les braises ne colmatent les espaces laissés 


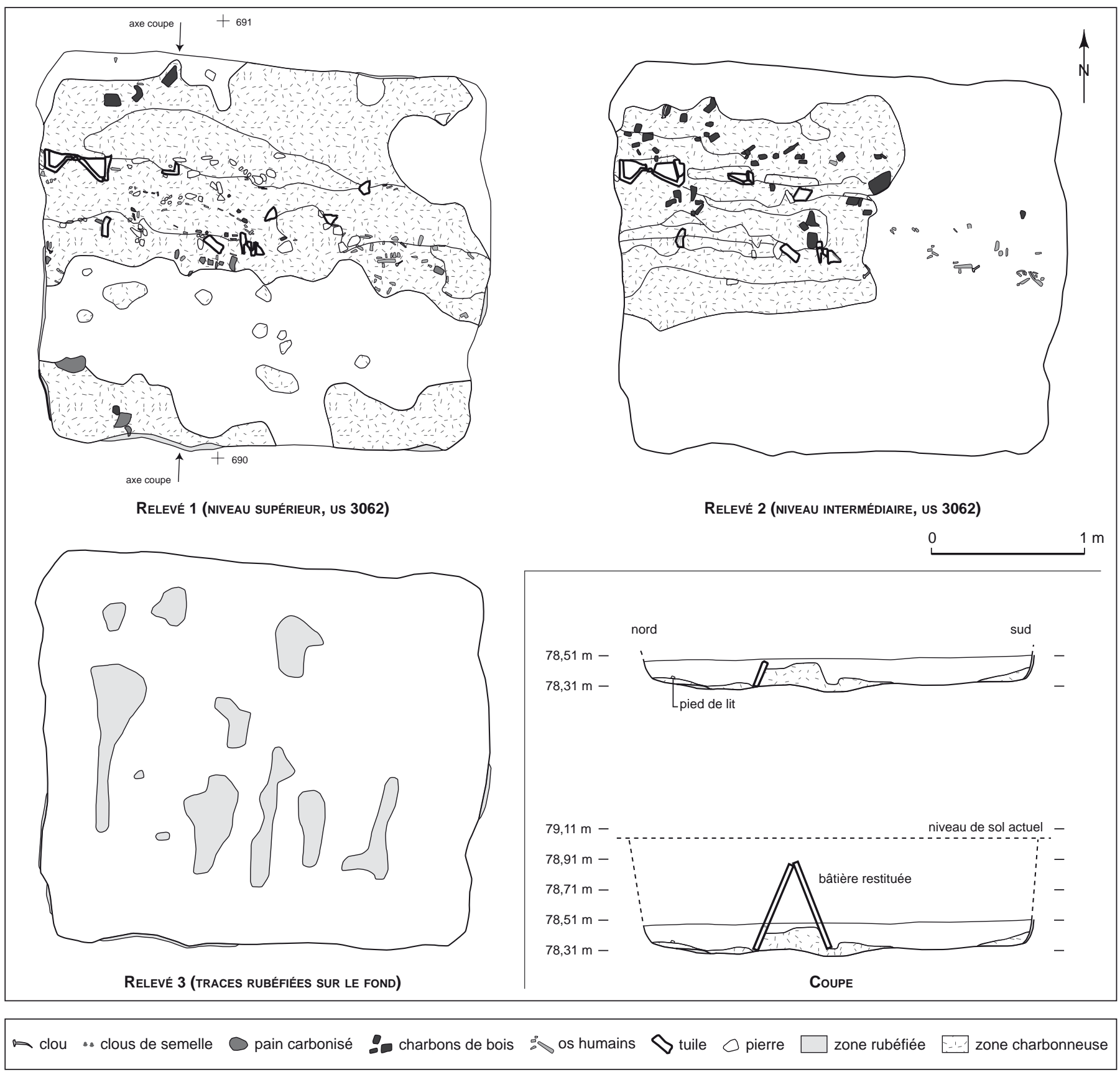

Fig. 81 - Le bûcher 3060 de Peyre Plantade à Clermont-l'Hérault, Hérault (DAO : A. Recolin, Inrap).

libres entre les bûches. L'expérimentation réalisée à AcyRomance (Ardennes) montre en effet que c'est au cours de cette phase initiale que l'aération de la structure est la plus importante et que la température de la base atteint son intensité maximale (Lambot, 1994).

On observe par ailleurs que ces traces rubéfiées occupent une emprise quadrangulaire de $2 \mathrm{~m}$ sur 1,85 m. Cette zone légèrement décentrée vers l'ouest est cernée par une bande non oxydée sur tout le pourtour de la fosse (de 0,30-0,40 m de largeur à l'ouest ; 0,20-0,30 m au nord ; environ 0,50 m à l'est et 0,35-0,45 $\mathrm{m}$ au sud). La partie médiane, construite sur une série de poutres parallèles, constitue selon toute probabilité la structure principale du bûcher. L'absence de marque d'oxydation le long des parois et sur leur partie 
inférieure invite à restituer un aménagement continu et peu élevé ceinturant la base du bûcher, peut-être pour le contrebuter ? L'hypothèse d'une conservation différentielle liée à l'intervention postérieure à la crémation et au ratissage des cendres peut être écartée dans la mesure où c'est précisément le long des parois, et notamment dans les angles, que se trouvent les principaux reliquats de la couche de crémation. L'épaisseur approximative des poutres peut être déduite de la largeur de l'espacement entre les bandes rubéfiées, de l'ordre de $0,20 \mathrm{~m}$ à $0,25 \mathrm{~m}$ environ. Ces données suggèrent l'existence d'une construction volumineuse dont la hauteur devait être au moins égale à la profondeur de la fosse. À partir de ces observations, on propose de restituer un bûcher construit, constitué de plusieurs rangées de bûches superposées entrecroisées, formant un massif de $2 \mathrm{~m}$ sur 1,85 m environ, d'une hauteur indéterminée d'au moins $0,60 \mathrm{~m}$.

En réalité, la rubéfaction renseigne surtout sur la manière dont la mise à feu a été effectuée, comme l'ont montré les crémations expérimentales et l'analyse des sédiments de bûchers archéologiques (Lambot, 1994 ; Pirling, 2002 ; Blaizot, Boës, 2003). Lorsque celle-ci s'effectue par le bas, le fond rougit puisque le feu se trouve à son contact, que l'air circule encore et que la chaleur est encore confinée dans la partie inférieure du bûcher. Par la suite, la température montant et le sol se trouvant en milieu réducteur du fait de l'accumulation de charbons qui jouent le rôle de bouclier thermique, la rubéfaction se porte sur le haut des parois, alors que la partie basse est caractérisée par des plages brunes indurées témoins d'une atmosphère réductrice. Il est à noter toutefois que la rubéfaction au sol n'est conservée que si la température a rapidement baissé à ce niveau, car les oxydes de fer ne résistent pas au-delà d'une température de $800^{\circ}$. C'est probablement ce qui s'est passé dans le bûcher de la Raude, à Tassin-la-Demi-Lune (Rhône) ; le fond, bien que largement entamé par deux cuvettes correspondant à des réaménagements de la couche de résidus, présentait une importante rubéfaction sur plusieurs centimètres d'épaisseur, de même que la banquette périphérique, située initialement à environ une vingtaine de centimètres au-dessus (fig. 82). Dans ce cas précis, on peut envisager que le bûcher prenait appui sur la banquette latérale, ce qui ménageait un emplacement pour le foyer tout en assurant une circulation d'air au sol. Des fosses à banquettes, rappelant ce dispositif, a été enregistrées sur le site de Krefeld-Gellep (Pirling, 2002, fig. 7-3, p. 499 et fig. 8-3, p. 500). Dans le bûcher du «Bois Baudry » (site de Pôle Synéo à Chartres, en Eure-et-Loir), daté de la seconde moitié du $\mathrm{II}^{\mathrm{e}} \mathrm{s}$. apr. J.-C. l'ustion in situ est corroborée par les traces de rubéfaction du sédiment encaissant au niveau des parois conservées sur 0,50 $\mathrm{m}$ de haut et du sol (épaisseur de quelques centimètres). Au regard des observations ethnologiques sur la conduite des bûchers, la rubéfaction du sol, signe d'une température élevée à la base du bûcher, pourrait être le signe d'un feu démarrant par le bas. La taille et la morphologie de la fosse (rectangulaire aux angles arrondis, à parois verticales et à fond plat, 4,34 m sur 2,58 m) permettent d'envisager un bûcher construit à l'intérieur.

Le dispositif décrit à la Raude, rappelle celui observé dans le bûcher 13 de l'ensemble funéraire méridional d'Aix-en-Provence, qui comporte une banquette de $0,35 \mathrm{~m}$ de largeur aménagée contre la paroi est de la fosse, une seconde pouvant être restituée du côté ouest (Nin et al., 2006, p. 178). Les bûchers pourvus ainsi d'un ressaut sont en réalité rencontrés ponctuellement à Lyon, mais on peut imaginer que l'arasement fréquent des parties hautes des structures minimise fortement ce type de dispositif. Toutefois, sur le site de la Favorite où les fosses sont conservées sur une bonne profondeur, une seule présente un double creusement (178). Aux 77-79 rue Pierre-Audry, la fosse 3041 possède un surcreusement central de près de 0,40 m. Ce dernier est comblé des résidus de crémation, mais on en trouve également sur le ressaut, ce qui montre bien qu'il servait de support au bûcher (fig. 83). Un bûcher du site de la station François-Verdier à Toulouse se présentait comme un double creusement : une plate-forme dans laquelle a été creusée une fosse réceptacle, qui contenait les résidus. Dans l'ensemble funéraire de Grand Noble 3 à Blagnac (Haute-Garonne), un dispositif identique a été mis en évidence.

\section{BÛCHER AU-DESSUS DE LA FOSSE (FOSSE-RÉCEPTACLE)}

Dans le cas des exemplaires étroits, on peut supposer au contraire que le bûcher était construit au-dessus de la fosse qui constituait alors le réceptacle des résidus. C'est ce que suggèrent les aménagements réalisés à la périphérie du bûcher 1004 de l'avenue Jean-Jaurès (voir supra, p. 103-104) ou bien les lambeaux de sols rubéfiés observés autour des bûchers à la Favorite (Tranoy, 1995b, p. 684-685). C'est également une construction aérienne qui a été restituée au col de Ceyssat, puisque le comblement de la séquence supérieure du bûcher débordait des limites de la fosse, attestant que l'édifice avait une emprise supérieure à celle de ce dernier (Trément, Humbert, 2005, p. 468-470). On peut supposer que pour les fosses à creusement en « V » 


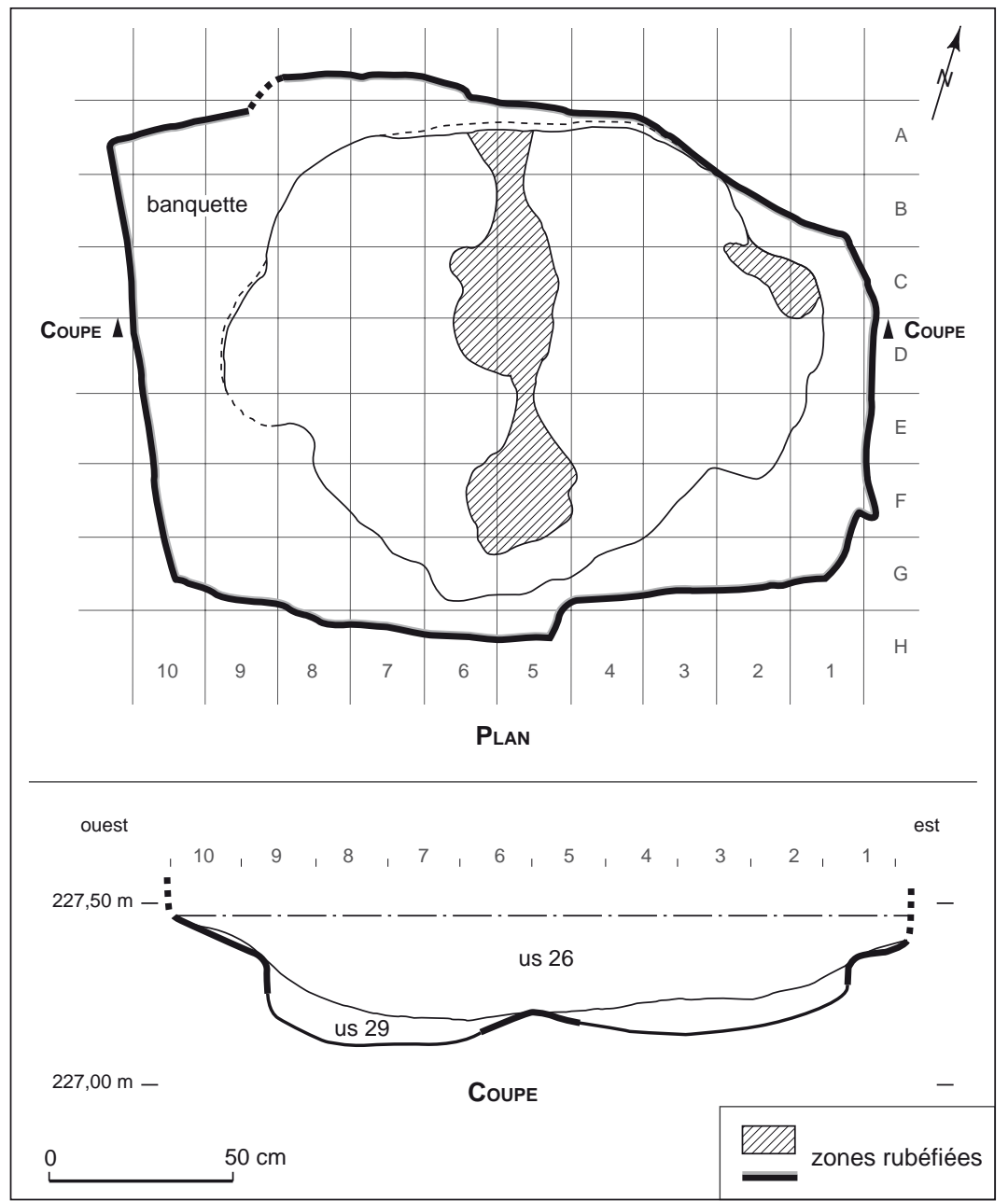

Fig. 82 - Le bûcher 23 de la Raude à Tassin-la-Demi-Lune, Rhône (relevé : J.-L. Gisclon, Inrap ; DAO : G. Macabéo, Inrap).

de Krefeld-Gellep mentionnées plus haut, le bûcher se trouvait de même au-dessus du sol.

La présence de bûches de bois de bonne taille incomplètement carbonisées à la base de la couche de crémation peut être l'indice d'un aménagement du bûcher au fond de la fosse, mais aussi d'un effondrement de la structure à l'intérieur avant que le bois ne soit complètement consumé. Ce phénomène a d'ailleurs été observé aussi bien dans les grandes fosses de Cagalou à Mouriès (Marcadal, Paillet, 2003, p. 273) que dans des bûchers de plus petits modules au Valladas (Bel et al., 2002, p. 92-93, en particulier dans la fosse B, les fosses 61, 105, 136, 137, 143, 146, 163, 209, 216, 262...), Cabasse (Bérard, 1961, p. 125 et p. 128-129), Sainte-Barbe (Moliner et al., 2003, pl. 23 et pl. 73, 2) et dans quelques-uns des bûchers de Vernègues (Chapon et al., 2004 , p. 143 , p. 146 , p. $162 \ldots)$.

\section{DISPOSITIFS PARTICULIERS}

Se pose aussi la question de la fonction des fragments de briques ou de tuiles, brûlés, parfois retrouvés sur le fond des fosses, généralement aux extrémités. Dans le bûcher 228 du Valladas (Bel et al., 2002, p. 344-345) et dans le bûcher 2249 du Mas de Vignoles IX à Nîmes (fouille inédite, V. Bel), ces éléments reposent sur la couche de résidus charbonneux conservée au fond des fosses. Dans le bûcher 247 du Valladas ou dans le bûcher 4077 de la Céreirède à Lattes, elles sont placées sur le fond de la fosse et recouvertes par des bûches ou des résidus de combustion (Bel et al., 2002, p. 401). Il n'est pas possible de déterminer si ces éléments ont servi pour l'agencement du bois, du mobilier ou du défunt. On note qu'au Valladas, ils sont situés à proximité de vases brûlés qui reposent sur le fond alors 


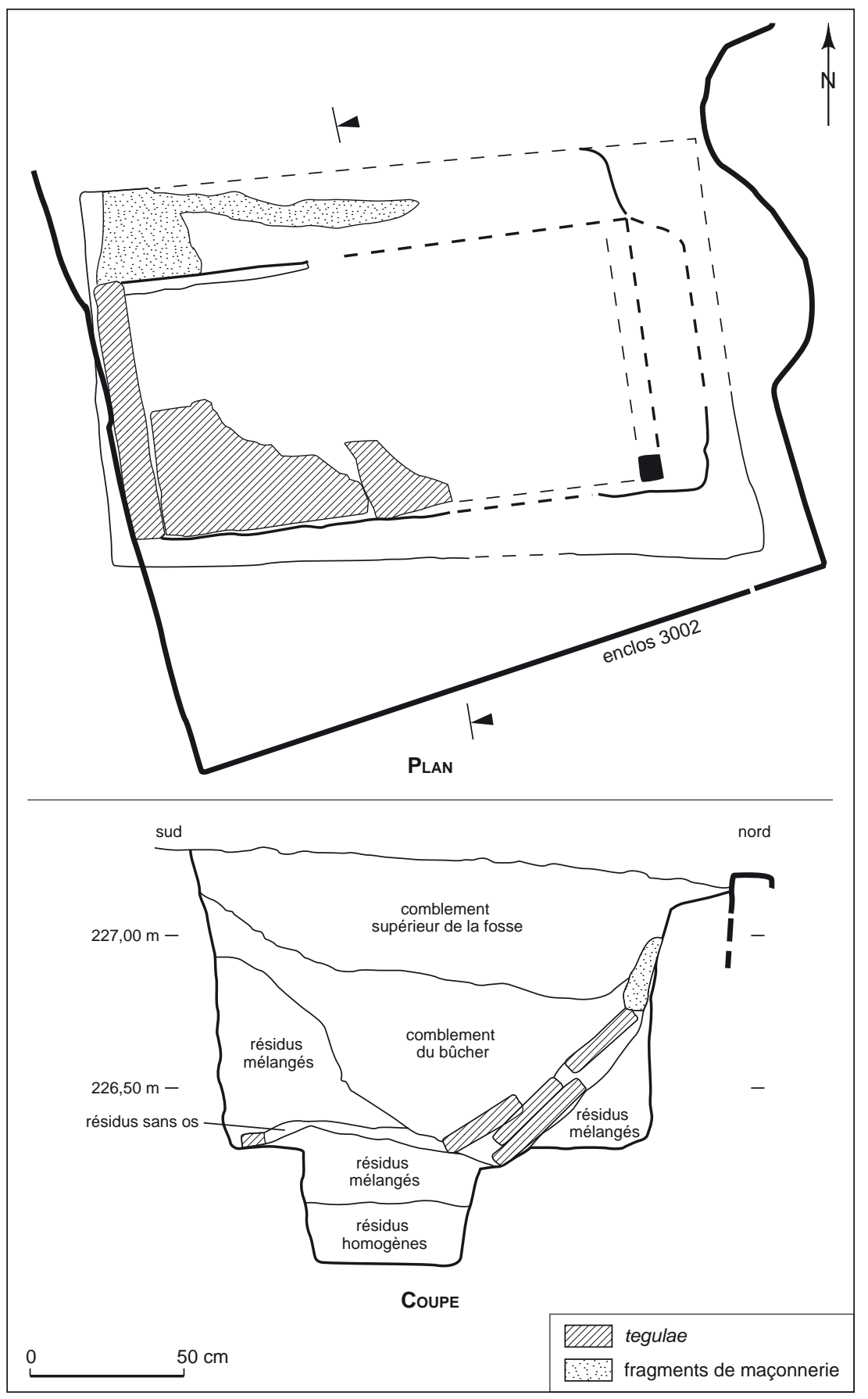

Fig. 83 - Le bûcher 3041 des 77-79 rue Pierre-Audry à Lyon (relevé : J.-L. Gisclon, Inrap; DAO : G. Macabéo, Inrap).

que les ossements humains sont concentrés dans la partie supérieure de la couche. Dans le bûcher 2249 du Mas de Vignoles (fig. 84), le dispositif comporte une tegula placée contre la paroi à l'extrémité de la fosse. La tegula légèrement brûlée est recouverte par des fragments d'un vase en céramique brûlé et des ossements humains, principalement du crâne. Une imbrex non brûlée et peut-être placée après la crémation surmonte l'ensemble. Sur la banquette du bûcher 3041 de la rue Pierre-Audry évoqué plus haut (fig. 83), deux tegulae sont placées verticalement contre la paroi aux deux extrémités de la longueur de la fosse (celle située à l'est est presque complètement détruite). La coupe indique que la 


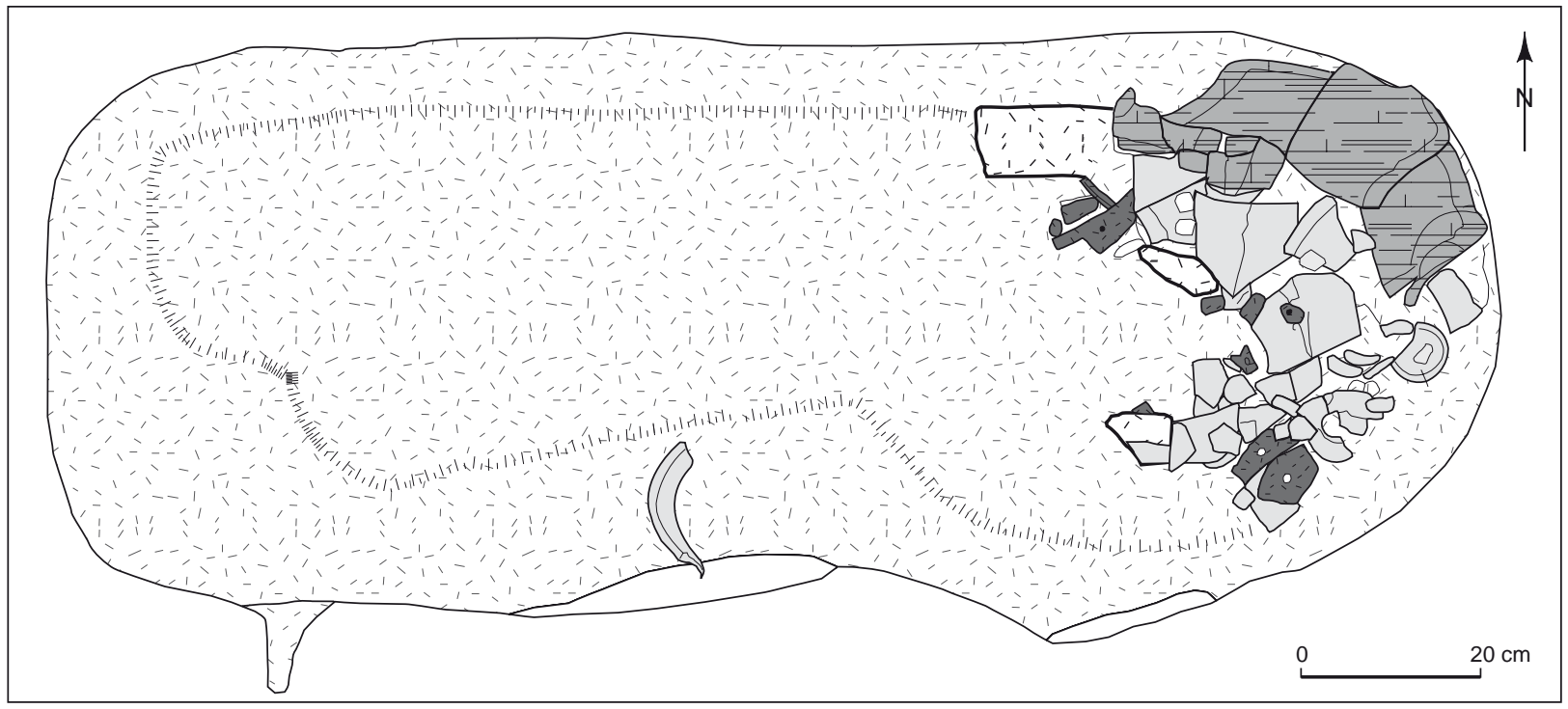

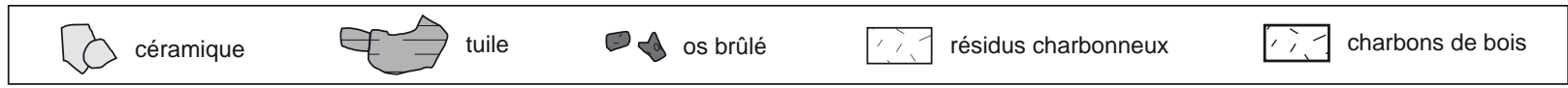

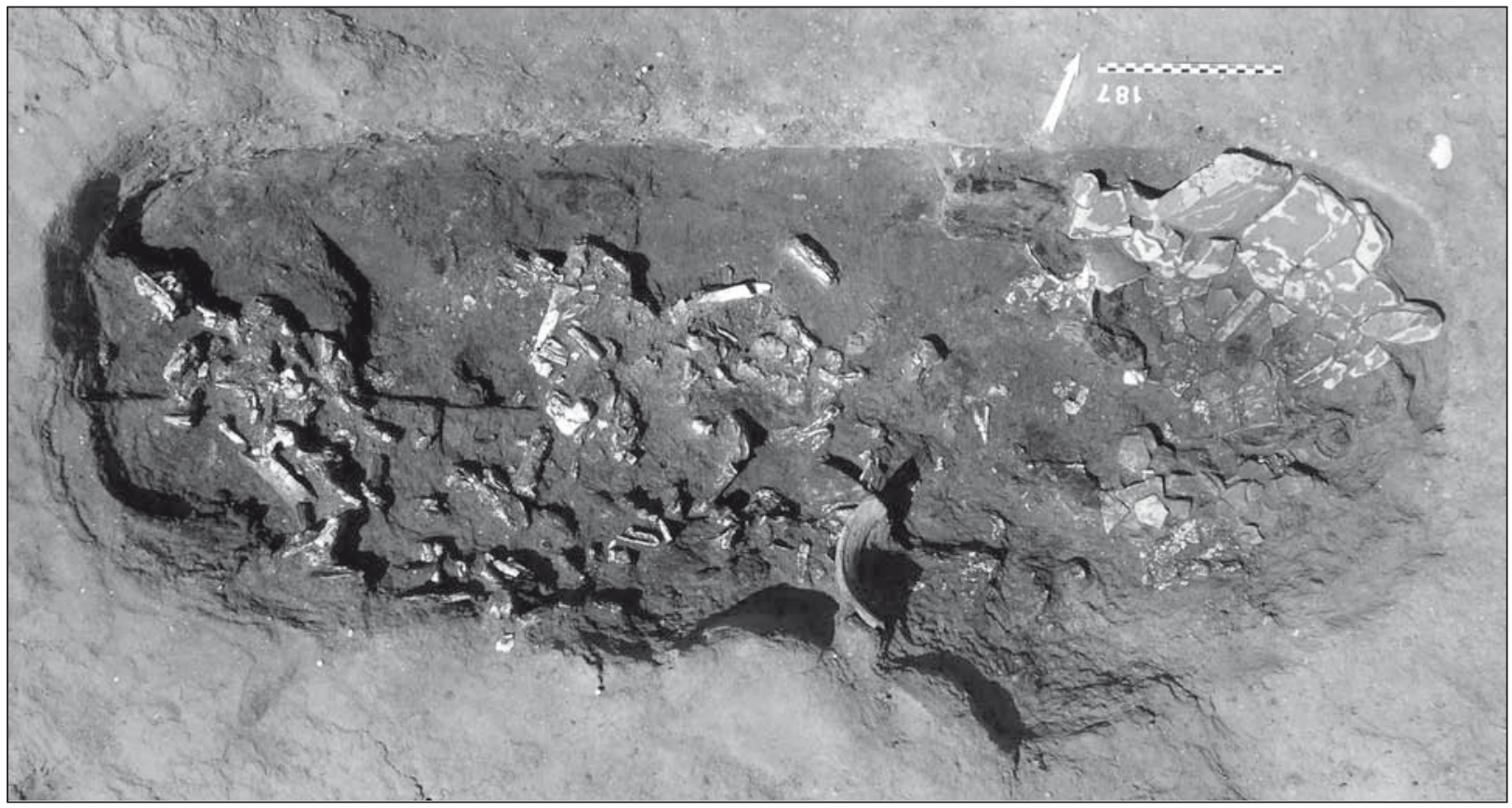

Fig. 84 - Aménagement de tuiles dans le bûcher 2249 du Mas de Vignoles IX à Nîmes, Gard (DAO : F. Robin et A. Recolin, Inrap; cliché : V. Bel, Inrap).

couche de crémation passe par-dessus ; d'autres tegulae, en revanche, reposent sur cette couche, dont elles suivent le pendage. S'agit-il, des vestiges d'une couverture installée a posteriori ou de la continuité de "l'habillage de la paroi » effondré après la crémation ? Les tuiles ne portent en tout cas aucune trace de brûlé, ce qui laisse envisager que le dis- positif est postérieur à la crémation, et donc que la totalité de la couche de résidus n'est pas en place.

Des pierres sont parfois associées aux résidus de crémation. C'est le cas d'une grosse pierre brûlée dans le bûcher 48 des Communaux de Saint-Cézaire (Chapon et al., 2004, p. 180), ou d'un fragment de bloc de marbre sculpté 
en remploi dans la tombe 2390 de l'Hôtel-Dieu à Narbonne, dans l'Aude (Ginouvez dir., 1996-1997, p. 128-129). On ne sait si ce dernier élément a subi l'action du feu ou s'il a été placé dans la fosse après la crémation, comme c'est probablement le cas du bloc rubéfié sur sa face inférieure découvert sur la couche de résidus du bûcher 2247 du Mas de Vignoles IX à Nîmes. Un dispositif plus élaboré a été mis en évidence dans le bûcher 71 des Communaux de SaintCézaire (Chapon et al., 2004, p. 193-194). Il est constitué de grosses pierres formant une sorte de coffrage et qui reposent sur chant, soit sur le fond de la fosse (pour les blocs parallèles aux petits côtés), soit sur la couche de résidus. Les restes osseux proviennent presque exclusivement des résidus situés à l'intérieur de cette structure interprétée comme un aménagement destiné à soutenir le bûcher (Chapon et al., 2004, p. 194).

Les couches charbonneuses conservées sur le fond des bûchers comportent bien souvent des fragments de terre cuite. Dans l'ensemble funéraire du Valladas, des amas de fragments de terre rubéfiée ont ainsi été observés au sommet de la couche de résidus et les restes osseux, principalement le long des parois (par exemple tombes 105, 127, 138, 195, cf. Bel et al., 2002, p. 324, 339, 459, 460). À Vers-Pont-du-Gard, des lentilles ou des nodules de terre cuite apparaissent dans la plupart des couches de crémation des bûchers. Ces inclusions sont parfois concentrées sur le pourtour de la fosse ou forment au sommet de la couche charbonneuse une couche mêlée de limons et de cendres (fig. 85). Parfois encore, elles sont localisées sur le fond et étroitement associées aux résidus de la crémation. Ces inclusions proviennent sans doute pour une part de l'effondrement des parois au cours ou à la fin de la crémation. Leur abondance, dans certaines des fosses de Vers-Pont-du-Gard, permet d'envisager l'existence d'une chape de terre recouvrant le bûcher à la manière d'une meule de charbonnier, ou bien un apport intentionnel de sédiment en fin de crémation pour arrêter la combustion. La première hypothèse paraît toutefois incompatible avec l'hétérogénéité de la coloration des pièces osseuses et avec les différents indices d'une bonne ventilation du bûcher, suggérée par la rubéfaction des parois et du fond.

C'est à des dispositifs d'ancrages de montants verticaux de ce type que pourraient correspondre les trous de poteaux mis au jour autour des fosses de crémations (fosses 12 et 40) de la ZAC Sextius-Mirabeau (Nin et al., 2006 , p. 176). La fosse 12 , de plan rectangulaire $(1,60 \mathrm{~m}$ x 1,20 m), est associée à deux trous de poteaux, implantés de part et d'autre de la fosse sur l'axe longitudinal médian. L'aire de crémation en fosse, F40, aménagée dans l'enclos 3, s'avère, quant à elle, encadrée par un dispositif de plan quadrangulaire de 2,50 m sur 3,50 m de côté, plus vaste que la fosse, identifié grâce aux négatifs de quatre poteaux $(0,15 \mathrm{~m}$ à $0,20 \mathrm{~m}$ de diamètre et $0,30 \mathrm{~m}$ à $0,40 \mathrm{~m}$ de profondeur). Il ne subsiste rien des poteaux, ce qui indique que la partie enterrée n'a pas été atteinte par la combustion. Il est impossible dans ce cas de déterminer si cette construction en bois est liée au bûcher lui-même (un plancher surélevé supportant le lit funéraire comme le propose l'auteur ou un système de contreforts) ou à l'aménagement de l'enclos après la crémation.

Un bûcher du site de la station François-Verdier à Toulouse était vraisemblablement, sinon construit, tout du moins aménagé. C'est en effet ce que suggèrent les empreintes semi-circulaires de rondins, placés verticalement contre les parois du creusement (limites externes de la plateforme), mais aussi autour du surcreusement interne.

Le bûcher de Champ Chalatras (II ${ }^{\mathrm{e}} \mathrm{s}$.), de forme quadrangulaire, s'avère avoir été flanqué de deux poteaux, implantés sur le fond de la fosse (fig. 86). Ils ont été placés à cheval sur les parois latérales du tiers sud de la fosse. Cette position et leur absence dans le tiers nord paraît désigner un dispositif «d'apparat » du bûcher (façade formant comme une porte ou, au contraire, un arrière-plan), plutôt qu'une conséquence de son édification et de sa stabilisation. Il ne s'agit en tout cas pas d'un aménagement a posteriori, puisque les résidus de crémation les occultaient complètement (les négatifs n'étaient visibles qu'au niveau du fond de la fosse).

\section{LA RÉPARTITION DES RESTES COMME TÉMOIN DU DISPOSITIF DU BÛCHER}

La répartition verticale des restes humains et du mobilier au sein de la couche charbonneuse contribue également à renseigner le dispositif du bûcher bien que, dans ce domaine, les observations ne soient pas toujours faciles à interpréter. On observe que les ossements sont généralement concentrés au sommet de la couche de résidus, plus rarement à sa base. Par exemple, pour ce qui concerne les bûchers 6200 et 6318 de Saint-Fréjus à Marennes, la plus grande partie des restes osseux a été retrouvée dans le premier niveau de décapage ( $80 \%$ pour le bûcher 6200 ), au sommet de la couche charbonneuse de 0,05-0,10 m d'épaisseur. Au Valladas, dans 12 cas sur 16, les ossements proviennent de la partie supérieure de la couche charbonneuse et dans 2 cas sur le fond (Bel et al., 2002, p. 94). Parfois des restes de branches carbonisées ont été retrouvées à la fois au-dessus et au-dessous des fragments osseux. Le bûcher 11172 de 


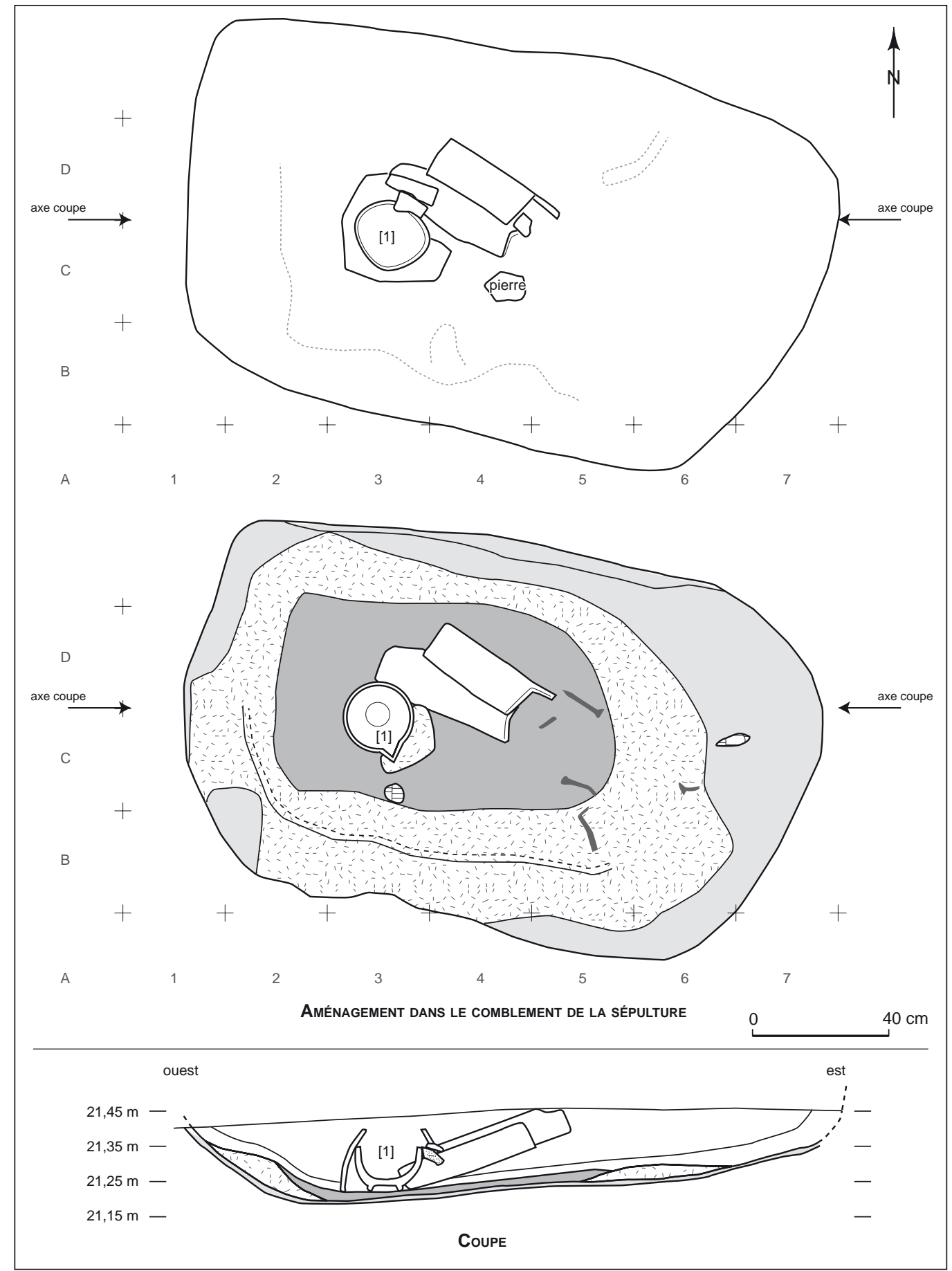

$\checkmark$ clou ……... saignée pelle $\square$ zone avec os humains $\quad \square$ zone rubéfiée $\quad \square$ zone charbonneuse

Fig. 85 - Le bûcher 10 de l'Estel à Vers-Pont-du-Gard, Gard (DAO : A. Recolin, Inrap). 


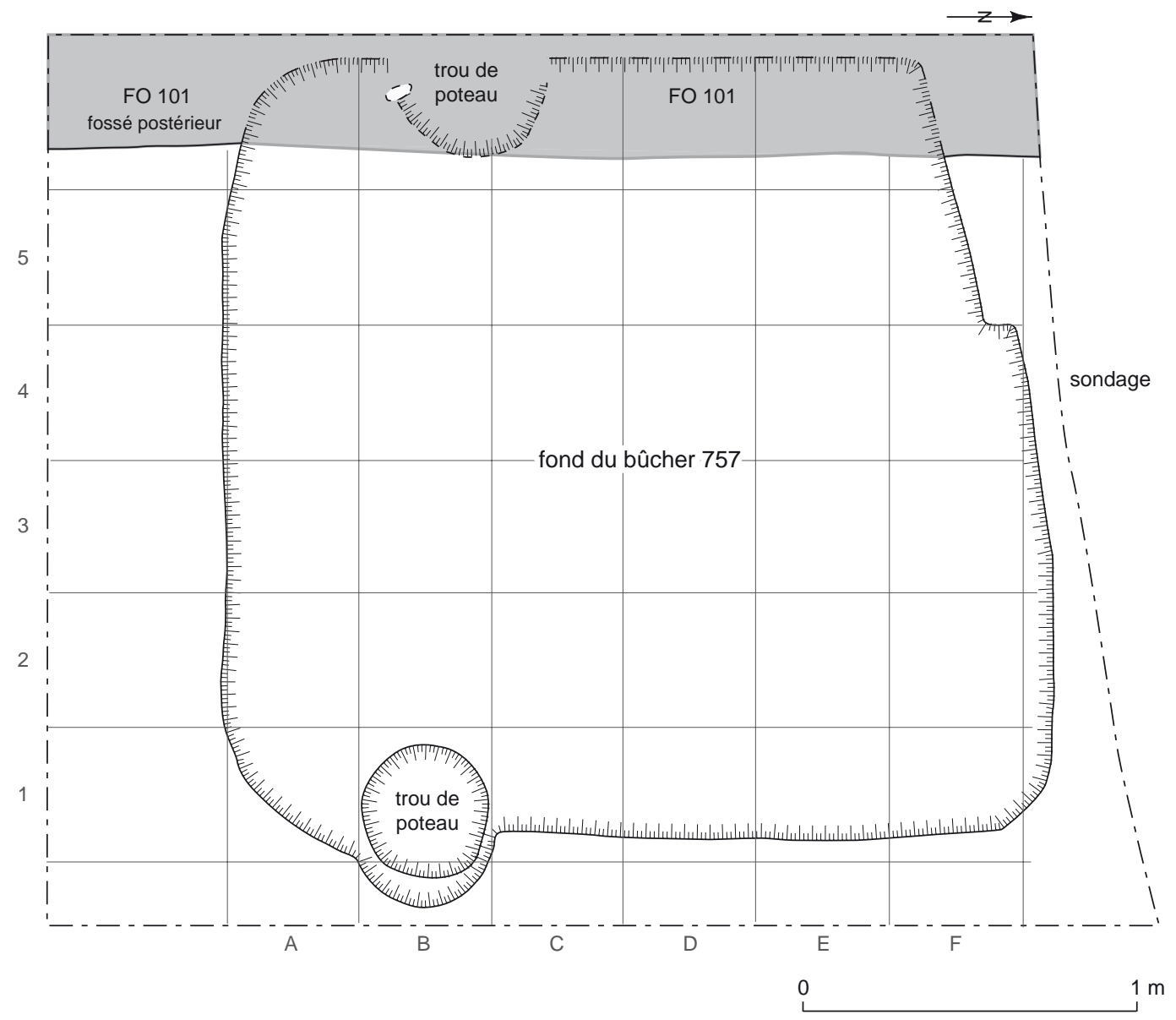

Fig. 86 - Aménagement de poteaux dans le bûcher 757 de Champ Chalatras aux Martres-d'Artière, Puy-de-Dôme (relevé : B. Rialland, Inrap; DAO : D. Parent, Inrap).

Soumaltre (Thernot et al., 2004, p. 316-317) présentait nettement deux couches, la première à la base très charbonneuse contenant de rares fragments d'os et de mobilier brûlés et de nombreux nodules de terre cuite, surmontée d'une seconde couche moins charbonneuse qui regroupait la quasi-totalité du mobilier brûlé et la plupart des restes osseux. Cette stratification se retrouve dans les bûchers du Mas de Vignoles IX. L'ensemble funéraire de Sainte-Barbe fournit des exemples où les os sont au contraire situés à la base de la couche de crémation (Moliner et al., 2003, p. 283, bûcher 26) et les bûches carbonisées en surface (Moliner et al., 2003, p. 353, bûcher 387). À Vers-Pont-duGard, les couches de crémation des bûchers en fosse sont peu épaisses (moins de $0,10 \mathrm{~m}$ ), et leur organisation varie beaucoup d'une tombe à l'autre. Dans un cas (bûcher 12), les fragments osseux sont le plus souvent situés à $1 \mathrm{~cm}$ ou $2 \mathrm{~cm}$ du fond. Dans trois autres bûchers (5, 11 et 13), les os ont été trouvés dispersés dans toute l'épaisseur de la couche de résidus de crémation, très charbonneuse dans le premier cas, très hétérogène dans le dernier. Enfin, dans deux bûchers (4 et 10), les restes osseux forment un amas dense associé à des cendres blanches, les résidus de charbons n'apparaissant que sur le fond ou contre les bords (fig. 85).

Il est fréquent qu'on ne puisse pas préciser le dispositif, mais à Lyon, dans tous les cas, le corps semble toujours avoir été placé au sommet du bûcher, aucun exemple ne venant à l'appui de l'hypothèse d'une crémation à l'étouffée. Sur le site du 62 avenue du Point-du-Jour, J.-L. Gisclon remarque toutefois que près de la moitié des ossements ont été retrouvés sous la couche de charbons, constituée en partie de brandons. Doit-on envisager ce mode de crémation ou bien un dispositif plus complexe, une véritable architecture crématoire, comme on en voit en Extrême-Orient, en partie effondrée sur les restes du corps?

Dans le bûcher 12 de la Grande Borne, à ClermontFerrand, la masse osseuse décroît en allant vers le fond de 


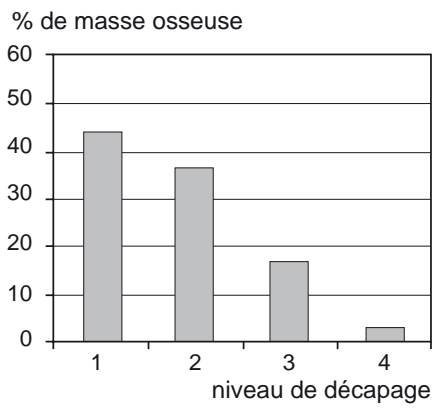

Bûcher 12

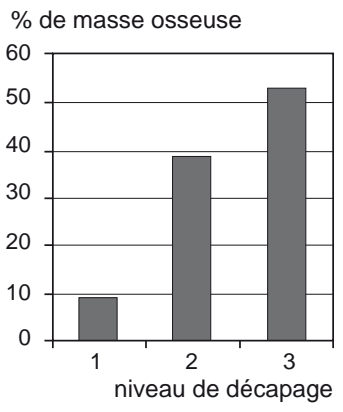

Bûcher 6
Fig. 87 - Distribution de la masse osseuse par niveaux de décapage des bûchers 12 et 6 de la Grande Borne à Clermont-Ferrand, Puy-de-Dôme (relevé : F. Blaizot, Inrap; DAO : G. Macabéo, Inrap).

la fosse ; par ailleurs, le crâne est mieux représenté sur le fond de la structure que dans deux premiers niveaux de décapage qui, en revanche, livrent la majorité des fragments se rapportant aux membres. La situation est à l'inverse dans le bûcher 6 : la masse osseuse est plus importante dans les niveaux inférieurs de la couche de résidus et la base comporte surtout les os des membres tandis que le crâne, les côtes et les vertèbres dominent les niveaux supérieurs (fig. 87). Les membres pesant plus lourd que ces éléments, il est probable que les valeurs relevées dans les niveaux inférieurs ou supérieurs de la couche de résidus varient en fonction des régions anatomiques qui s'y trouvent. Ces différences de masse se rapportent ainsi à l'ordre dans lequel les diverses parties du squelette se sont effondrées. La même interprétation peut être proposée pour le bûcher 58 du 54 rue Pierre-Audry à Lyon : dans la moitié nord de la fosse, la majorité de la masse osseuse provient des niveaux supérieurs de la couche de résidus alors que dans la moitié sud, les os sont concentrés sur le fond; la répartition verticale différentielle des $1841 \mathrm{~g}$ d'os provient vraisemblablement du mode d'effondrement du bûcher.

La variabilité des situations apparaît donc très importante. Ces données peuvent être mises en relation avec la situation relative du corps et du bois ou avec celle du bûcher lui-même (dans ou au-dessus de la fosse), avec la taille de la fosse, avec le mode d'effondrement du bûcher, ou encore avec des pratiques ayant pour objectif d'activer la combustion pendant la crémation, voire avec les activités postcrématoires (voir chapitre III).

\section{LE CHOIX DES ESSENCES ET DU TYPE DE BOIS}

L'étude des essences de bois utilisées pour la crémation et de leur répartition dans la couche de résidus permet parfois d'aller plus loin dans la restitution du bûcher, mais dans ce domaine, les séries disponibles sont encore trop peu nombreuses, les analyses anthracologiques étant encore rarement mises en œuvre dans les opérations d'archéologie préventive. Dans les régions méditerranéennes, à notre connaissance, seuls les ensembles du Valladas (Bel et al., 2002, p. 511-515), de Soumaltre (Thernot et al., 2004, p. 262-264) et du Mas de Vignoles IX (étude inédite de I. Figueiral) ont fait l'objet d'études systématiques. Dans les ensembles du Valladas (64 structures étudiées dont 35 bûchers) et d'Aspiran (25 tombes dont 3 bûchers), ce sont les chênes (chêne vert et chêne pubescent au Valladas ; chêne Quercus serpervivens à Soumaltre), qui dominent dans les assemblages, aux côtés du Pin sylvestre ou Pin d'Alep à Aspiran, et du Hêtre au Valladas. En revanche, l'étude de I. Figueiral montre que les deux bûchers du Mas de Vignoles IX ne conservaient aucun fragment de Chêne, ni de Pin ni de Hêtre, les essences représentées (9 taxons identifiés) provenant de bois d'eau (Orme, Peuplier) ou de fruitiers (vigne, figuier, noyer...). Ces études font surtout ressortir la grande variabilité taxinomique de l'approvisionnement en bois des bûchers, vraisemblablement en fonction de l'environnement immédiat de l'espace funéraire : 20 taxons, regroupant une trentaine d'espèces, ont été reconnus à Soumaltre (Thernot et al., 2004, p. 263) ; 23 taxons au Valladas (Bel et al., 2002, p. 511). À Soumaltre, comme au Valladas, les bûchers et les tombes les mieux documentés livrent 6 à 10 taxons. Dans la publication du site de Sainte-Barbe (Moliner et al., 2003), les seules mentions concernent le Pin d'Alep et, exceptionnellement du Cyprès (bûcher 378) et du chêne pubescent (bûcher 80), mais il s'agit de déterminations effectuées sur des échantillons ponctuels, souvent prélevés sur des clous (par exemple dans les bûchers 80, 81, 113, 136, 138).

Le faible nombre d'analyses anthracologiques rend difficile les comparaisons, voire la mise en évidence de tendances. Pour le Sud-Ouest, l'ensemble funéraire de la Vayssière à L'Hospitalet-du-Larzac semble être le seul site du Haut-Empire sur lequel ce type d'étude a été effectué. Les structures étudiées comportaient en majorité soit du chêne pubescent, soit du Hêtre (Fourteau-Bardaji et al., 1993, p. 268).

L'analyse des charbons de bois du bûcher des Vernes à Faverdines (Cher) a été menée par D. Marguerie. Son rapport indique qu'il a été élevé presque exclusivement avec du Chêne (sessile ou pédonculé), sous la forme de fragments de tronc ou de grosses branches, dans le but de donner un feu très calorifique mais lent, qualités qui lui 
Tabl. XI - Sélection des combustibles sur le site des 77-79 rue Pierre-Audry à Lyon (F. Blaizot, d'après les données d’Archéolabs).

\begin{tabular}{|c|c|c|c|c|c|c|c|c|c|c|c|c|c|c|c|c|c|c|c|c|c|c|}
\hline Espèces & $\begin{array}{c}\text { DP10 } \\
84\end{array}$ & \begin{tabular}{|c|} 
DP10 \\
85
\end{tabular} & \begin{tabular}{|c|} 
DP10 \\
93
\end{tabular} & \begin{tabular}{|c|} 
DP11 \\
17 \\
\end{tabular} & \begin{tabular}{|c|} 
DP11 \\
41
\end{tabular} & \begin{tabular}{|c|} 
DP11 \\
42
\end{tabular} & \begin{tabular}{|c|} 
DP20 \\
52
\end{tabular} & \begin{tabular}{|c|} 
DP30 \\
07
\end{tabular} & \begin{tabular}{|c|} 
DP12 \\
5
\end{tabular} & $\begin{array}{c}\text { DP13 } \\
4\end{array}$ & \begin{tabular}{|c|} 
DP13 \\
5
\end{tabular} & \begin{tabular}{|c|} 
DP15 \\
1
\end{tabular} & \begin{tabular}{|c} 
DP13 \\
8
\end{tabular} & \begin{tabular}{|c|} 
DP14 \\
1
\end{tabular} & $\begin{array}{c}\text { DP14 } \\
6\end{array}$ & $\begin{array}{c}\text { DP15 } \\
9\end{array}$ & \begin{tabular}{|c|} 
DP20 \\
1
\end{tabular} & \begin{tabular}{|c|} 
DP10 \\
19
\end{tabular} & \begin{tabular}{|c|} 
BUF5 \\
9
\end{tabular} & \begin{tabular}{|c|} 
BUF1 \\
70
\end{tabular} & $\begin{array}{c}\text { BUF3 } \\
138\end{array}$ & $\begin{array}{c}\text { BUF3 } \\
146 \\
\end{array}$ \\
\hline \multicolumn{23}{|l|}{ Chêne } \\
\hline \multicolumn{23}{|l|}{ Hêtre } \\
\hline \multicolumn{23}{|l|}{ Frêne } \\
\hline \multicolumn{23}{|l|}{ Sapin } \\
\hline \multicolumn{23}{|l|}{ Pin } \\
\hline \multicolumn{23}{|l|}{ Érable } \\
\hline \multicolumn{23}{|l|}{ Saule } \\
\hline \multicolumn{23}{|l|}{ Aulne } \\
\hline \multicolumn{23}{|l|}{ Noisetier } \\
\hline \multicolumn{23}{|l|}{ Fusain } \\
\hline Prunus & & & & & & & & & & & & & & & & & & & & & & \\
\hline
\end{tabular}

sont reconnues (Fourteau-Bardaji et al., 1993, p. 268). Il est probable aussi selon l'auteur qu'il s'agissait vraisemblablement de l'espèce la plus courante. D'autres études, que l'on peut qualifier d'anecdotiques, comme sur le site des Mahis à Gy-en-Sologne (Loir-et-Cher), ne permettent pas véritablement d'aller au-delà de ces conjectures.

Des différences ont parfois été observées entre la partie inférieure et le sommet de la couche charbonneuse. Ainsi à Cabasse, dans le bûcher 15 de la Calade, une couche de terre fumigée contenant des débris charbonneux de brindilles et de feuillages de Chêne vert (Quercus ilex) et de Clématite des haies (Clematis vitalba) surmontait une seconde couche charbonneuse qui couvrait de grosses branches carbonisées posées sur le fond (Bérard, 1961, p. 125). L'étude de L. Fabre sur les restes de bois de l'ensemble funéraire de Soumaltre fait également apparaître une répartition verticale différenciée des taxons, dans la couche de résidus charbonneux du bûcher 11172 (Thernot et al., 2004, p. 321). Dans ce cas, la partie la plus profonde se distingue par la présence d'éricacées (famille des bruyères) et la partie supérieure par celle de pins (sylvestre/salzmann), de pomoïdées et de chênes caducifoliés, les chênes (Quercus sp.) et la vigne (Vitis vinifera) apparaissant dans les deux horizons. L. Fabre émet l'hypothèse que ces deux couches pourraient refléter la composition du bûcher dont la partie inférieure serait composée de bois de petit calibre (éricacées, vigne et chênes verts) et la partie supérieure de bois de pins, de chênes caducifoliés, de fagots de pomoïdées et de vigne. «À l'image d'un foyer, le bois d'allumage a pu reposer en dessous du bois de comburage. Lors de la crémation, des rajouts de petit bois (recherche de flamme) ont pu être réalisés ». Cette même étude montre en outre que les espèces arbustives représentent plus de $30 \%$ des prélèvements. Le fait qu'il y ait beaucoup d'espèces représentées (voir supra, p. 114) et que celles-ci soient des essences naturellement arbustives, implique des conditions particulières pour que la calcination se produise. Cela conduit à envisager l'hypothèse d'une utilisation de fagots comme combustibles, sachant qu'un fagot de petit bois présente les mêmes caractéristiques thermiques qu'une bûche (Thernot et al., 2004, p. 265). Ce mode d'approvisionnement pourrait être lié au caractère rural de l'ensemble funéraire de Soumaltre, bien qu'au Valladas, les espèces naturellement arbustives soient également bien représentées. Ajoutons que la présence de sarments de vigne à la base du bûcher a également été observée dans une tombe de la proche campagne nîmoise, au Mas de Vignoles IX (étude en cours).

Les rares séries de la région Rhône-Alpes ayant fait l'objet de ce type d'étude concernent trois sites lyonnais seulement, ceux des 54 et 77-79 rue Pierre-Audry et celui du 62 avenue du Point-du-Jour (étude Archéolabs de 1992). Au 54 rue Pierre-Audry, aucun des résidus des six bûchers examinés ne livre plus de cinq taxons : le Hêtre est sur-représenté, et s'accompagne du Sapin (cinq cas), du Frêne, du Chêne, de l'Orme (un cas chacun), ainsi que de noisetier, de buis et de pignes de pin. Aux 77-79 rue Pierre-Audry, chaque structure ne livre pas plus de quatre taxons à la fois (tabl. XI). On constate l'omniprésence du Hêtre : sur vingt-deux structures examinées en effet, une seule n'a livré que du Chêne, tandis que dans toutes les autres, le Hêtre domine, en nombre, sur les autres fragments. Dans neuf cas, le Hêtre est associé au Chêne, dans deux cas au Frêne, dans trois cas au Sapin ; 
le Pin, l'Érable, le Saule et l'Aulne ne sont présents qu'une fois. Enfin, le Noisetier est rencontré dans trois structures, le Fusain dans deux et le Prunus dans une. Bien entendu, ces résultats sont fonction de l'état de conservation des charbons et des modalités de prélèvement. On ne peut pas toujours interpréter tous les résultats : si, à l'échelle d'une série, la présence massive de Hêtre, de Pin, de Chêne, etc., permet de restituer la composition fondamentale du bûcher, certains bois prélevés sur des arbres fruitiers ont peut-être été utilisés pour faire des objets ou des meubles (coffres, étagères, cercueil...) qui composaient le mobilier funéraire. Rue Pierre-Audry notamment, le saule qui n'est représenté qu'une fois dans des résidus de crémation, a été utilisé dans la construction du cercueil d'une inhumation (tombe 1124) contemporaine des crémations sur le même site. Au 54 rue Pierre-Audry, où le Hêtre domine, des fragments de Buis pourraient se rapporter à un objet tourné. La question est également posée à la Grande Borne à propos de fragments de Noyer issus d'un seul secteur d'un bûcher (étude de M. Cabanis, Inrap). La fonction de la pigne de pin, relativement fréquente, est encore discutée : la présence simultanée des pignons et des écailles est-elle l'indice d'un usage des pignes comme matériau combustible ou au contraire comme dépôt alimentaire (Bel et al., 2008) ? En Auvergne, l'étude des combustibles provenant de plusieurs structures funéraires antiques est en cours par M. Cabanis dans le cadre de son doctorat. Sur le site de la Grande Borne, dont l'étude des résidus de sept structures est terminée, une importante variabilité est observée : le Hêtre est majoritaire dans trois structures (63-92-97 \% des charbons), le Frêne (94\%), le Chêne $(86 \%)$ et le Sapin $(87 \%)$ ne prédominant qu'une fois chacun. Dans toutes les structures cependant, le Chêne, le Hêtre et le Sapin sont systématiquement associés. On trouve aussi ponctuellement du Prunellier, des Pomoïdés et de l'Érable, qui ont sans doute servi à allumer le feu. Sur les sites de Champ Bussière et Champ Lamet (période antique) à Pont-du-Château (Puy-de-Dôme), le Hêtre domine, le Chêne et le Sapin étant secondaires ; il est possible que le Sapin blanc, piètre combustible, ait été employé comme bois d'œuvre dans les bûchers.

Les différentes études réalisées jusqu'à présent semblent indiquer qu'une sélection était opérée parmi les espèces en présence, par site ou par structures (Fourteau-Bardaji et al., 1993, p. 268-269), ce dont témoigne effectivement la confrontation de nos séries. En effet, si en l'état du corpus actuel, les ensembles lyonnais ou auvergnats paraissent privilégier le Hêtre, il arrive qu'un ou plusieurs bûchers d'une série comportent des espèces différentes ou bien identiques à la majorité de ceux de la même série, mais en proposition inverse. Dans les ensembles méridionaux, l'approvisionnement des bûchers apparaît très diversifié et pourrait être qualifié d'opportuniste, ceci même lorsque les analyses montrent une certaine sélection, qui peut être simplement due au fait que l'essence en question est, sinon la seule, tout du moins la plus courante, voire la plus efficace. Ces conclusions devront être validées par de nouvelles analyses et surtout par une étude conjointe de l'approvisionnement des bûchers et celui des habitats contemporains les plus proches.

\section{CONSTRUCTION ET DÉCORATION DU BÛCHER}

La construction et la décoration des bûchers sont fréquemment évoquées en raison de la présence, souvent abondante, d'éléments de tabletterie et de clous, mais aussi d'artefacts métalliques d'un autre type, la plupart du temps indéterminés à cause de l'ustion et de la fragmentation. Ces aspects peuvent aussi être appréhendés à partir de la forme même du creusement ou des traces laissées dans le sol. Mais, dans l'un comme dans l'autre cas, il est difficile, sinon impossible, de déterminer ce qui relève de la construction ou de la décoration du bûcher. Est-ce parce que ces deux aspects sont étroitement liés?

La fréquence et l'abondance des clous d'assemblage en fer caractérisent la plupart des couches de résidus charbonneux Au Valladas, trente-six bûchers sur trente-huit ont livré des clous d'assemblage, soit un total de 1289 clous auxquels s'ajoutent 774 clous issus des résidus des dépôts secondaires. Certains renfermaient plusieurs centaines de fragments, comme le bûcher 258 qui a livré 382 fragments correspondant à un minimum de 150 clous d'assemblage (Bel et al., 2002, p. 156 et p. 434), ou le bûcher 58 du 54 rue Pierre-Audry qui compte 312 clous en NMI. Le bûcher de Voiron en comportait 65, répartis sur toute la surface, avec ponctuellement des concentrations particulières (fig. 88). Dans l'ensemble funéraire des Communaux, les bûchers ont livré de 2 à 82 clous (Chapon et al., 2004, p. 133), tandis que l'ensemble funéraire de Soumaltre en a fourni 217 pour 24 tombes (Thernot et al., 2004, p. 259).

La présence de ces clous a fait l'objet de diverses interprétations dans la littérature archéologique. L'hypothèse de l'utilisation de bois de récupération afin de procéder à l'ustion est parfois évoquée, en particulier quand les clous sont peu nombreux et/ou de formes différentes. La diversité des essences utilisées (voir supra, p. 114-116) pourrait refléter une utilisation de bois recyclé, tout autant 


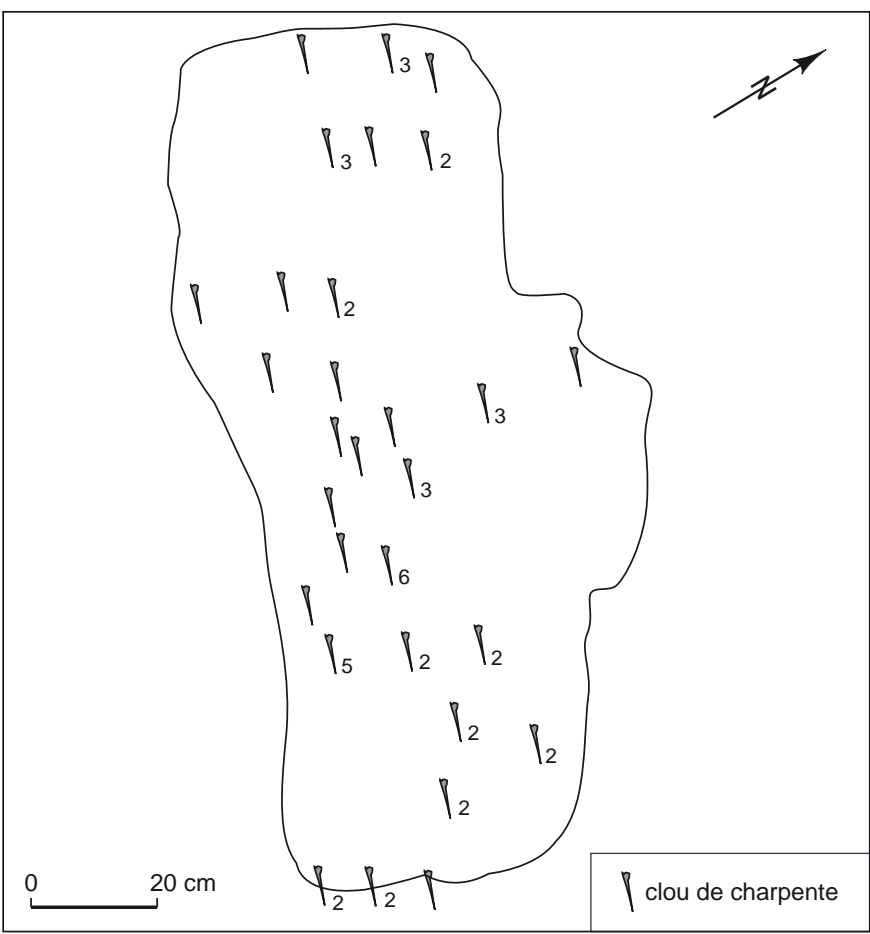

Fig. 88 - Répartition des clous de charpente du bûcher 25 de la Brunerie à Voiron, Isère (relevé : F. Blaizot, Inrap; DAO : G. Macabéo, Inrap).

qu'un approvisionnement opportuniste constitué d'espèces prélevées dans le proche environnement. La question se pose toutefois pour le Hêtre qui a pu être utilisé pour l'ameublement ou la charpente. Il apparaît très régulièrement dans les crémations en contexte méditerranéen comme au Valladas (Bel et al., 2002, p. 514-515) ou à Aspiran (Thernot et al., 2004, p. 302), alors qu'il est normalement inféodé à des contextes plus montagnards. Une origine locale n'est toutefois, pour l'heure, pas du tout exclue. La présence éventuelle d'élément d'assemblage de charpente, comme celui retrouvé à une extrémité du bûcher 8 des Communaux (Chapon et al., 2004, p. 149) ne peut pas non plus être retenue comme preuve de l'utilisation de bois de réemploi.

On peut penser en effet que de telles pièces ainsi qu'une bonne partie des clous, notamment les plus grands, ont pu servir à la construction ou à l'habillage du bûcher. Selon l'hypothèse de M. Feugère (Thernot et al., 2004, p. 259), les clous de grands modules peuvent avoir servi à fixer des planches sur les montants ou des planches entre elles et les clous de petite taille avoir été utilisés pour fixer sur la structure du bûcher des éléments décoratifs ou symboliques. Le bûcher 12 de la Grande Borne conserve 83 clous. L'étude réalisée par L. Orengo (Archéodunum) a montré que certains sont pourvus d'une tige droite mais que d'autres ont leur extrémité distale rabattue. Ces derniers sont majoritaires aux abords des parois de la fosse et se concentrent plutôt dans la partie supérieure de la couche de crémation. Il est ainsi permis de supposer que les clous droits ont servi à assembler les bûches, tandis que les clous à extrémité rabattue étaient destinés à fixer plusieurs pièces de bois d'épaisseur régulière, vraisemblablement des planches de $10 \mathrm{~mm}$ d'épaisseur à des tasseaux de même épaisseur, de façon à confectionner des panneaux. Ces derniers ont pu être disposés de manière à parementer le bûcher en façade ou bien être placés horizontalement, fixés sur des montants verticaux, afin de constituer un dispositif supportant ou contenant le défunt, et/ou recevant le mobilier sur plusieurs étages.

De nombreux bûchers ont livré ces grands clous, parfois qualifiés « de charpente ", dont la section de la tige, sous la tête, mesure entre $5 \mathrm{~mm}$ et $10 \mathrm{~mm}$, dont la longueur varie entre $80 \mathrm{~mm}$ et $110 \mathrm{~mm}$. Des éléments métalliques, également de grande taille, de type "agrafe », font parfois aussi partie de ces lots. Il existe par ailleurs une multitude d'autres clous, nettement plus petits, mais de taille et de forme diverses. Cette diversité est telle que les synthèses à leur sujet sont fastidieuses à réaliser. Si l'on peut facilement les distinguer des clous de chaussure, il est en revanche malaisé de classer les fragments de clous ; à l'issue de l'examen, la question de leur fonction (édification du bûcher, décoration, éléments du contenant ou autre) reste posée. C'est sans doute pourquoi on rencontre beaucoup d'études dans lesquelles les clous sont certes traités, mais sans définir de catégories (hormis pour les clous de chaussure). Leur localisation au sein du bûcher permet parfois d'émettre des hypothèses.

Le site lyonnais du Sextant, rue du CommandantCharcot, qui a fonctionné durant tout le $\mathrm{I}^{\mathrm{er}}$ s., se caractérise par la faible fréquence des clous de construction et de menuiserie. En effet, une large majorité d'individus (99\%) est de petite taille (section quadrangulaire de $2 \mathrm{~mm}$ à $4 \mathrm{~mm}$ de côté) et correspond à ce que J.-P. Guillaumet définit comme des clous de tapissier (Guillaumet, 2003, p. 62-63). Les clous de taille moyenne (dont la section est comprise entre $4 \mathrm{~mm}$ et $8 \mathrm{~mm}$ ), utilisés en menuiserie, sont peu fréquents. Relevés uniquement dans quatre structures, ils représentent moins de $1 \%$ de la totalité des fragments découverts. Enfin, un unique clou de construction est issu d'un dépôt mixte de crémation, et pourrait provenir de bois de récupération. Le nombre de clous issus des bûchers n'est pas uniforme. Quatre d'entre eux contiennent entre 150 et 200 clous, une en contient 27 tandis que les autres en livrent 
entre 40 et 90 . La quasi-absence de clous de charpente et de menuiserie permet d'aborder les techniques de construction des bûchers sur ce site. Il semble en effet évident que les bûches furent assemblées sans clous, ce qui implique un dispositif d'édification du bûcher différent de celui mis en ouvre sur d'autres ensembles funéraires lyonnais ; la rareté des grands clous est également soulignée dans les bûchers de Krefeld-Gellep, en Rhénanie (Pirling, 2002, p. 520). Quant aux petits clous, largement majoritaires, ils ont sans doute servi à la décoration du bûcher (couronnes, guirlandes, tentures), cette hypothèse étant étayée par leur dissémination dans la fosse. Sur le site de Krefeld-Gellep, ils sont interprétés comme provenant des cercueils, des civières ou des lits funéraires (id., ibid., p. 520).

\section{LE DISPOSITIF DU CORPS}

\section{LA POSITION DU CORPS SUR LE BÛCHER}

Dans la majeure partie des cas, on se heurte à de grandes difficultés pour restituer la position du corps sur le bûcher et il arrive même que l'on soit dans l'incapacité de déterminer son orientation. En effet, non seulement les membres fléchissent naturellement sous l'action du feu, mais en outre les régions anatomiques sont très fréquemment brassées par certaines pratiques destinées à activer la combustion du corps, ou encore par des remaniements qui interviennent en fin de crémation au moment de la récupération des os (voir chapitre III). Si l'on examine les 21 bûchers provenant de la région Rhône-Alpes, dans lesquels la couche de crémation est intacte et qui ont fait l'objet d'un enregistrement in situ approprié, 11 ne livrent aucun renseignement sur l'orientation du corps, et les autres ne fournissent que l'orientation générale. Dans le bûcher 30 du 54 rue Pierre-Audry, où les fragments du crâne, du tronc et des membres supérieurs sont majoritairement dans la moitié est et ceux des membres inférieurs dans la moitié centre-ouest, la répartition différentielle des fragments d'os longs latéralisés pourrait indiquer que le corps reposait sur le ventre, à moins qu'un retournement ne se soit produit pendant la crémation (fig. 89). Dans le bûcher 293 du Replat, à Aime, toutes les régions anatomiques sont réparties de manière relativement égale dans les diverses parties de la fosse ; la petite taille de cette dernière $(1,10 \mathrm{~m})$ comparativement à celle, moyenne, que l'on peut attribuer à l'adulte qui fut brûlé dans ce bûcher, pourrait être à l'origine de ce brassage apparent (fig. 90). On peut en effet imaginer

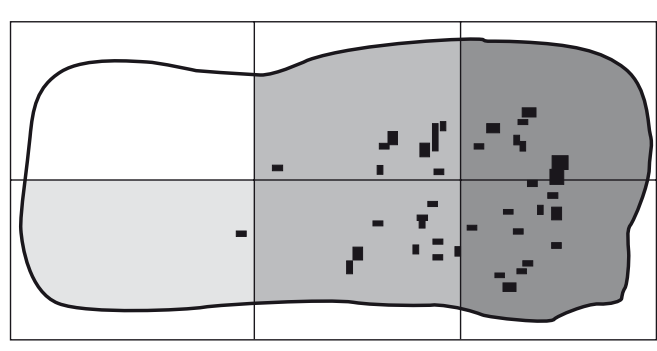

Fragments de CRÂNE DE LA TOMBE 30

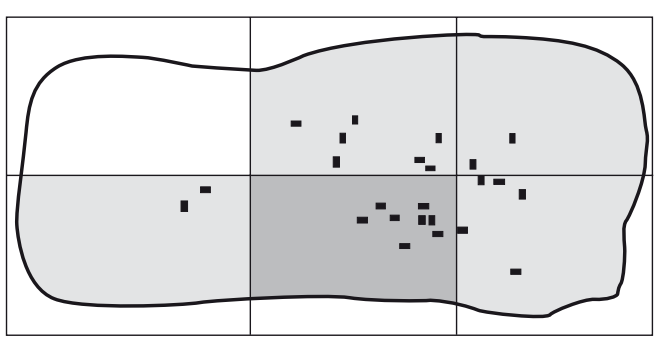

Fragments de tRonC DE LA TOMBE 30

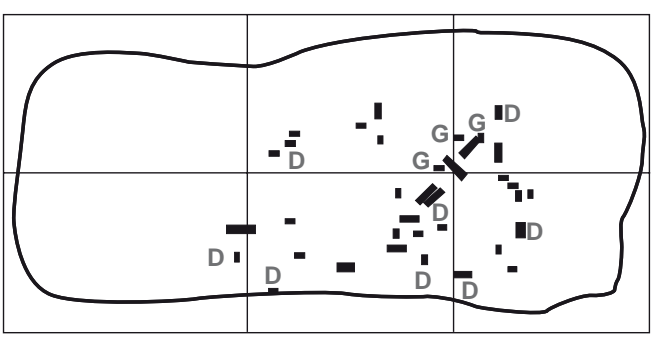

FraGmENTS DE MEMBRES SUPÉRIEURS

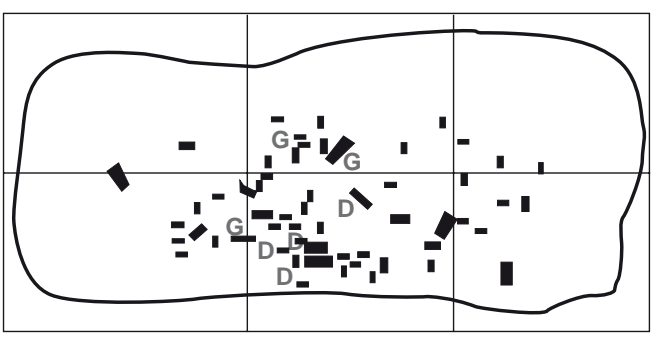

FrAGMENTS DE MEMBRES INFÉRIEURS

0

- gros fragments

D fragments droits

G fragments gauches

Fig. 89 - Répartition spatiale des régions anatomiques dans le bûcher 30 du 54 rue Pierre-Audry à Lyon (relevé : V. Bel, Inrap; DAO : G. Macabéo, Inrap).

que le corps était fléchi et que ce brouillage correspond à l'effondrement d'un bûcher sur lequel le corps était ramassé 


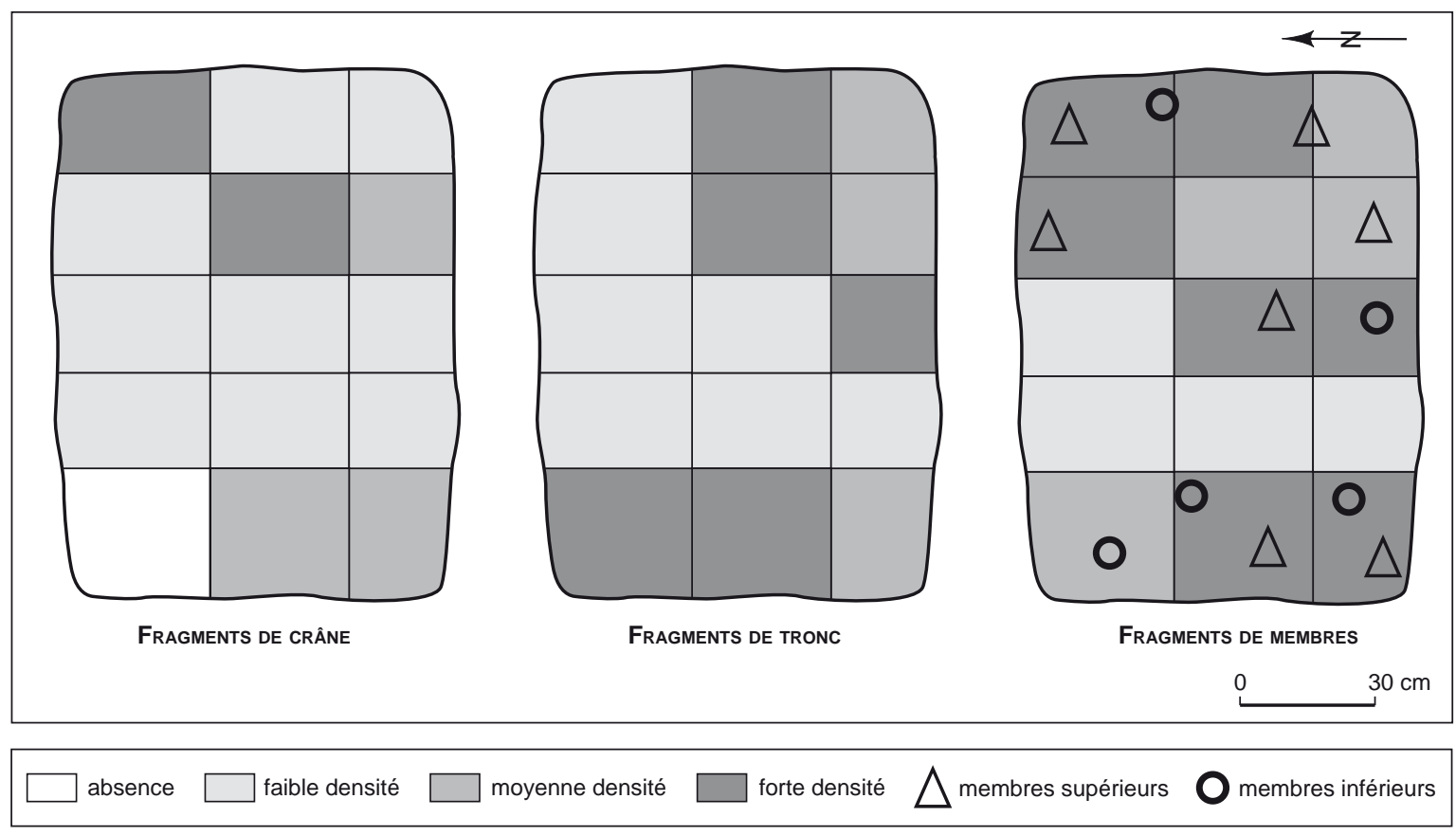

Fig. 90 - Répartition spatiale des régions anatomiques du bûcher 293 du Replat à Aime, Savoie (relevé : F. Blaizot, Inrap ; DAO : G. Macabéo, Inrap).

sur lui-même, ou bien qu'il était étendu sur un bûcher placé au-dessus de la fosse qui servait avant tout de foyer et qu'en fin de crémation, les résidus brûlés dispersés sur le sol ont été reversés dans la fosse. La seconde hypothèse semble argumentée par la mise en évidence de quatre couches de comblement, de la plus riche (sur le fond) à la plus pauvre (au sommet conservé) en os, en matériel et en charbons.

Dans les régions méditerranéennes, on dispose à ce jour d'une série de 80 bûchers pour lesquels la position générale du corps a pu être documentée (16 exemplaires en Languedoc et 64 en Provence dont 45 à Vernègues et 16 à Marseille, Sainte-Barbe). En Languedoc, l'orientation générale a pu être définie dans 14 cas dont 6 pour lesquels il a été possible, à partir de la latéralisation des pièces osseuses, de préciser que le sujet, en fin de crémation, se trouvait sur le dos. Dans la plupart des bûchers provençaux étudiés la position des corps n'est pas lisible en raison des « manipulations » effectuées en cours de crémation ou de déplacements opérés à l'issue de celle-ci. À Vernègues, parmi les 45 exemplaires documentés, 7 seulement ont livré des indices suffisant pour restituer la position générale du corps, auxquels s'ajoutent 9 autres cas à Marseille et un à Bollène (site du Pont de Pierre). Lorsque les données sont suffisamment explicites (quatre exemples seulement en Provence), il semble que le corps était placé sur le dos.
L'orientation du corps dépend bien évidemment de celle de la fosse. Le plus souvent, lorsqu'elle est cohérente d'un point de vue anatomique, la répartition des restes osseux indique que l'axe du corps coïncide avec l'axe longitudinal de la fosse, parfois cependant avec un décalage sur l'un des côtés, les ossements n'occupant qu'une moitié de la fosse. C'est le cas par exemple du bûcher de Gallière à Montpellier (voir infra, p. 124-126) dans lequel les restes osseux étaient circonscrits à la moitié est de la fosse orientée nord-sud. Deux bûchers de grandes dimensions (6318 du site de SaintFréjus à Marennes et 11104 du site de Soumaltre) présentent une organisation différente. La distribution des pièces semble indiquer que le corps a été placé sur un côté de la fosse perpendiculairement à son axe longitudinal.

La documentation disponible montre une très grande diversité des situations : toutes les orientations sont illustrées et cette variabilité s'observe au sein d'un même site. Les bûchers de l'ensemble funéraire de l'Estel, les mieux documentés sont tous orientés est-ouest et les corps sont placés tête à l'est (un cas) ou à l'ouest (trois cas) (fig. 91). À Sainte-Barbe, les corps brûlés sur des bûchers nord-sud ont été placés tête au sud (trois cas) ou au nord (quatre cas), tandis qu'à Vernègues, où les fosses sont orientées nordouest/sud-est, la tête a été retrouvée au nord-ouest dans six cas sur sept. Il serait plus intéressant de connaître la dispo- 


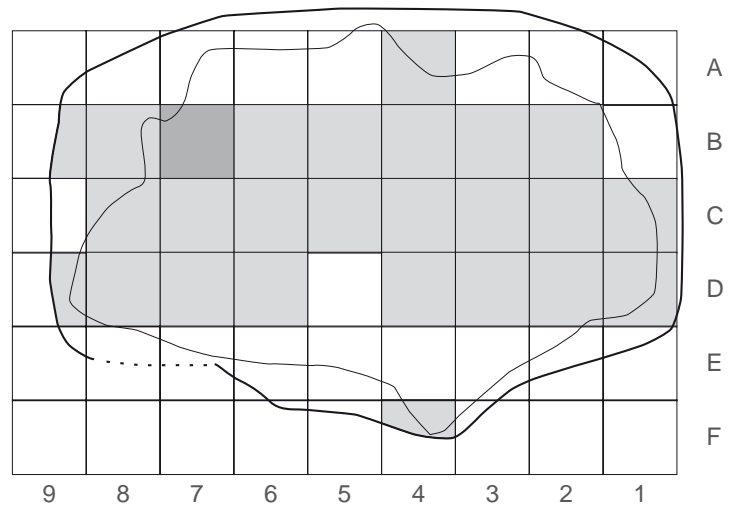

Os BRÛLÉS HUMAINS

poids des os localisés : $1841,9 \mathrm{~g}$

poids total : $1893,3 \mathrm{~g}$

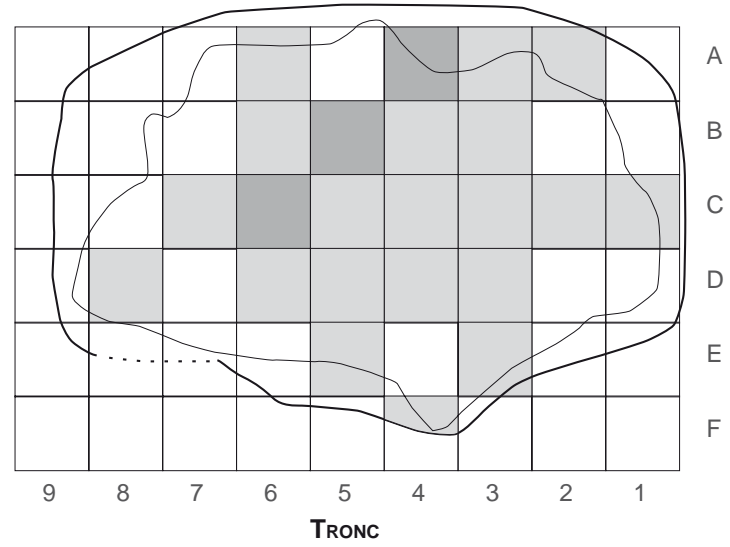

poids des os localisés : 182,4 g

poids total : $184,4 \mathrm{~g}$

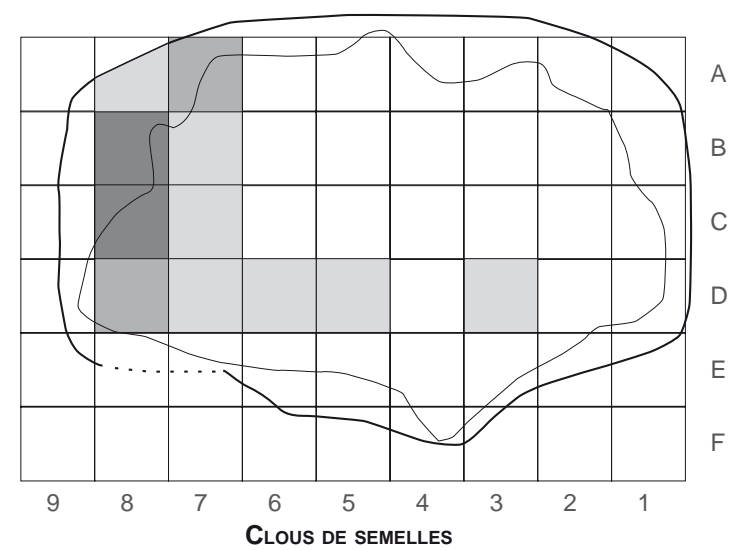

poids des os localisés : 422,1 g

poids total : $433,8 \mathrm{~g}$

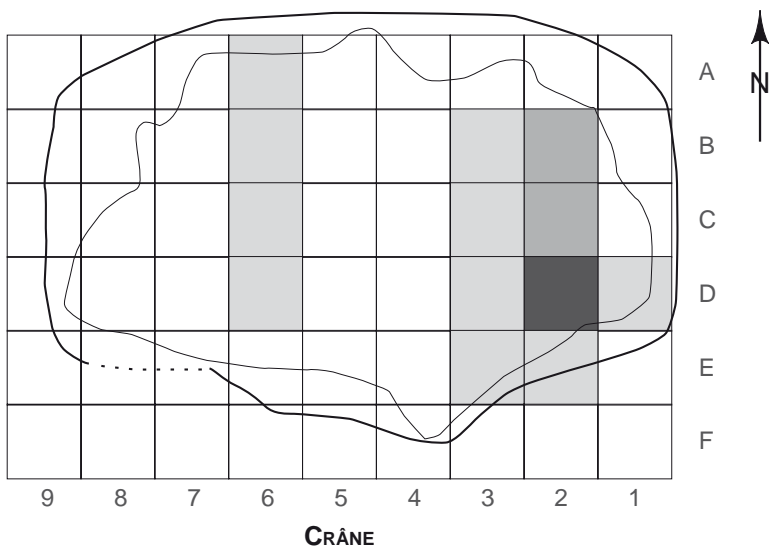

poids des os localisés : $237,7 \mathrm{~g}$ poids total : $247,2 \mathrm{~g}$

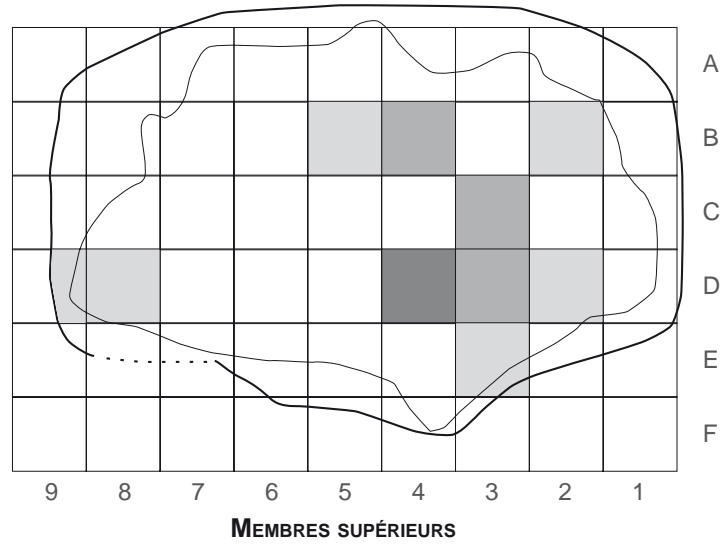

poids des os localisés : 147,9 g

poids total : 153,1 g

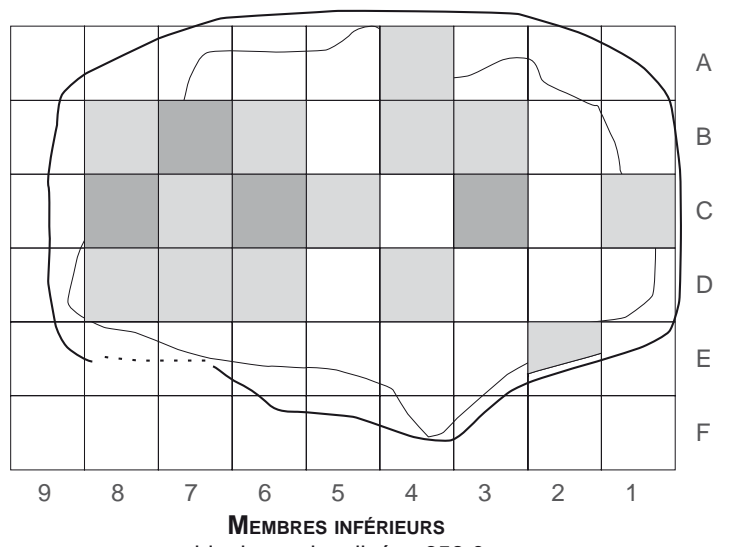

poids des os localisés : 658,0 $\mathrm{g}$

poids total : 655,1 $\mathrm{g}$

\begin{tabular}{|l|l|l|l|l}
\hline & $-1 \%$ & $\square, 1-10 \%$ & $\square$
\end{tabular}

Fig. 91 - Répartition spatiale des régions anatomiques dans le bûcher 5 de l'Estel à Vers-Pont-du-Gard, Gard (DAO : A. Recolin, Inrap). 


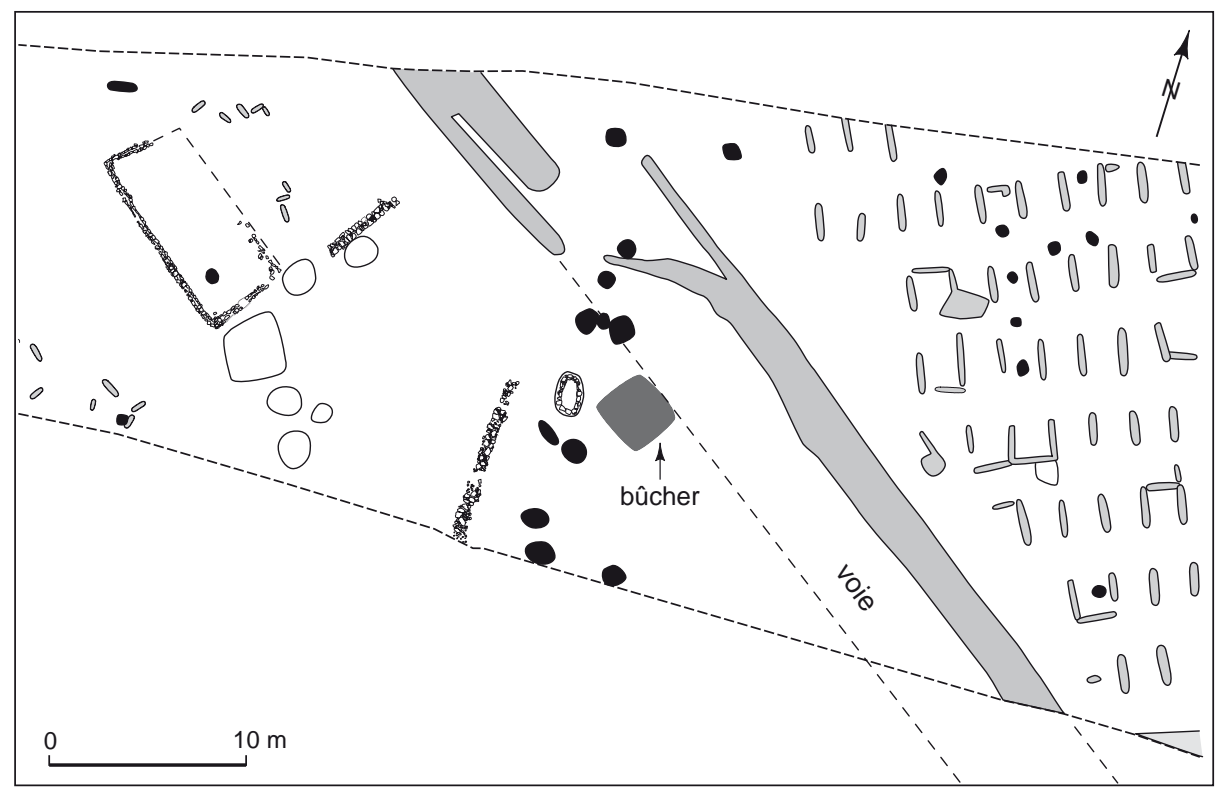

Fig. 92 - Localisation du bûcher 3060 de Peyre Plantade à Clermont-l'Hérault, Hérault (DAO : A. Recolin, Inrap).

sition du corps et l'organisation du bûcher par rapport au espaces de circulation, mais on dispose en ce domaine de très peu d'exemples. La grande fosse de crémation mise au jour aux abords de l'agglomération de Peyre Plantade est implantée à $5 \mathrm{~m}$ à l'ouest d'une voie d'accès à l'habitat, de direction est-ouest (fig. 92). Au sein de la fosse quadrangulaire, parallèle à la voie, la répartition des restes osseux indique que le sujet avait été disposé perpendiculairement au chemin, la tête à l'est (du côté de la voie) et les pieds à l'ouest. Cette disposition s'observe également sur le site du Mas de Vignoles IX, implanté le long d'une voie. L'un des bûchers en fosse est parallèle au chemin, mais le second est perpendiculaire et contient les restes d'un sujet disposé tête à l'est, côté voie.

\section{DISPOSITIFS DE PRÉSENTATION DU CORPS SUR LE BÛCHER}

Parmi les nombreux clous retrouvés dans les couches de résidus de crémation certains ont pu être utilisés pour l'assemblage du cercueil ou bien du support sur lequel reposait le défunt (voir supra, p. 116-118). Cette interprétation est suggérée par la présence de divers éléments métalliques (charnières de coffre, bandages de renfort, pieds de lit ou meuble...), ou de pièces de décoration (appliques en os, clous décoratifs en bronze...). Les exemples sont nombreux. Au Valladas, le bûcher 195 renfermait sur le fond des fragments de bandage de renfort en fer dont la disposition suggère de les attribuer à un coffre de bonne taille qui a pu servir de cercueil d'autant que la plupart des ossements brûlés ont été trouvés à proximité de ces éléments (Bel et al., 2002, p. 339) (fig. 93). Sur ce site (id., ibid., p. 156-157), les clous robustes, utilisés pour l'assemblage des cercueils (section supérieure à $4 \mathrm{~mm}$, longueur comprise entre $60 \mathrm{~mm}$ et $160 \mathrm{~mm}$, exemplaires coudés assemblant des épaisseurs de bois de plus de $35 \mathrm{~mm}$ ) étaient bien moins représentés dans les crémations que les clous de taille moyenne (section de 3-4 mm, longueur comprise entre $25 \mathrm{~m}$ et $80 \mathrm{~mm}$, clous assemblant des épaisseurs de 15-40 mm seulement). Quelques lots font exception comme celui du bûcher 195 qui fournit par ailleurs des éléments de coffre. Les clous de taille moyenne sont également prédominants dans les structures de l'ensemble funéraire méridional d'Aix-en-Provence (Nin et al., 2006, p. 117). Les clous les plus nombreux, moins robustes, pourraient donc avoir eu d'autres fonctions (habillage du bûcher, assemblage de supports légers, civières ou meubles). Les 90 clous associés à des éléments caractéristiques de lit dans le bûcher de Peyre Plantade appartiennent à cette catégorie. En revanche, les éléments de lit du bûcher 6200 de Saint-Fréjus (étude inédite, V. Bel) étaient associés à 77 exemplaires robustes, 180 exemplaires de taille moyenne et 138 exemplaires de petite taille. Il apparaît donc bien difficile de préciser le type de dispositif utilisé à partir de la seule clouterie. D'autres éléments d'assemblage particuliers, comme les clavettes associées à Saint-Fréjus (bûcher 6200) à des éléments de lit, et présents également 


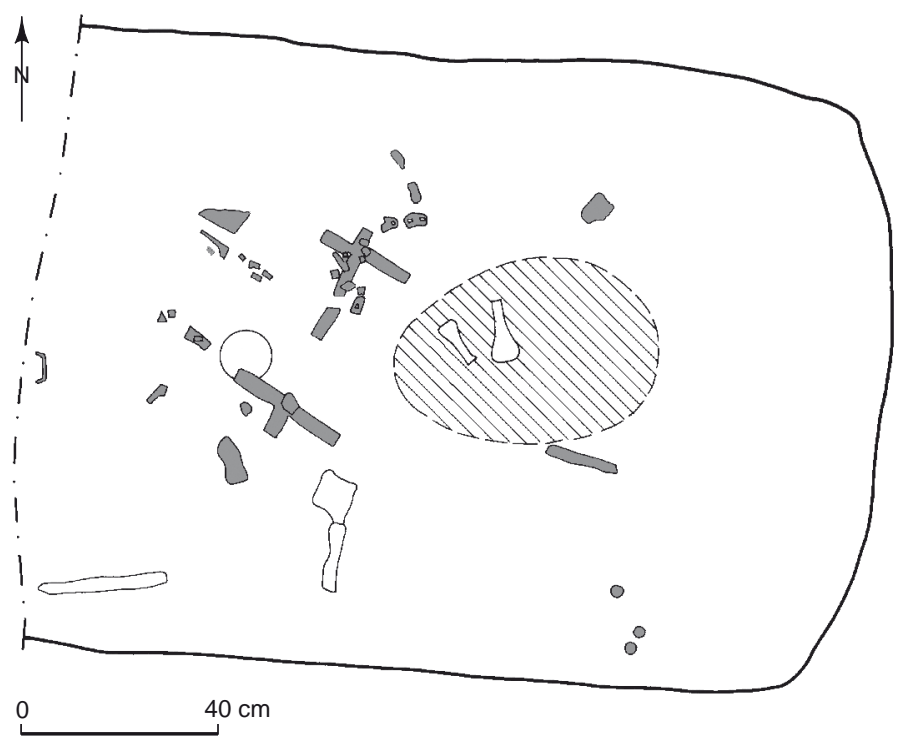

Fig. 93 - Éléments de renfort de coffre dans le bûcher 195 du Valladas à Saint-Paul-Trois-Châteaux, Drôme (dessin : M.-N. Baudrand).

dans les bûchers de Vernègues (Chapon et al., 2004, p. 133) pourraient être attribués à des meubles. Selon M. Feugère, le clou en $\mathrm{T}$ du bûcher 65 des Communaux de Saint-Cézaire à Vernègues appartient à un type de couchette rustique " éventuellement assemblée pour le seul transport du mort sur le bûcher » (id., ibid., p. 133).

À Voiron, les clous de taille moyenne de type «tapissier ", à tête plate et à la pointe très effilée, sont moins nombreux que les clous de charpente, mais suivent la répartition de ces derniers S'il reste difficile d'en déterminer la fonction, ils sont manifestement liés soit au décor du bûcher soit au dispositif du corps.

L'existence de lits est bien attestée grâce aux éléments de placages en os ou en ivoire caractéristiques (Nicholls, 1979 ; Béal, 1986 et 1991) et par des pièces d'assemblage en fer.

Sur l'ensemble funéraire rural de Saint-Fréjus, des vestiges de lit et/ou de meuble ont été relevés dans trois bûchers et dans une structure de dépôt. Le bûcher 6200 comportait un lot de 45 pièces d'appliques en os de décor de pied et de cadre de lit associées à des clavettes en fer, ainsi qu'à trois grandes tiges robustes en fer $(300 \mathrm{~mm}, 295 \mathrm{~mm}$ et $250 \mathrm{~mm}$ de longueur) et aux fragments d'une quatrième, caractérisées par une extrémité massive à section carrée précédée par une pièce carrée et interprétées comme des armatures de pieds de lit (ou de meuble).

Le bûcher 6318 renfermait trois (ou quatre) pieds de lits en bronze, en forme de sabots et des pièces de renfort en bronze, associés à deux tiges en fer similaire à celles de la tombe 6200 . Le bûcher 6100 a fourni quatre tiges robustes en fer, munies d'une extrémité massive en forme prismatique de section carrée et sur lesquelles sont enfilées des rondelles en fer (fig. 94). Enfin, le dépôt de crémation 6319 a livré une armature de pied de lit (fig. 94) ainsi que des éléments d'assemblage et plusieurs renforts en tôle de fer comportant des trous de fixation. Plusieurs plaques, coudées, correspondent à des renforts d'angle. L'une des faces de ces pièces présente des restes de tissus fossilisés par l'oxyde métallique ou bien des restes de bois et de matière organique brûlés. Ils étaient associés à de nombreux clous en fer dont 19 petits clous à tête hémisphérique et 120 petits clous à tête plate portant des traces de tissus sous la tête. Ces différents éléments suggèrent de restituer un lit ou une banquette recouverte de tissus maintenus par des plaques en fer et par les clous de tapissier.

Des clous décoratifs (en bronze cette fois), sont également attestés à Peyre Plantade en association avec des éléments de décor de lit en os, ainsi qu'aux Communaux (Chapon et al., 2004, p. 133). Les tiges en fer apparaissent dans plusieurs autres contextes avec des variantes concernant la forme des extrémités et la longueur des tiges. On signale ainsi trois exemplaires avec clous en bronze et pièces de tabletterie dans le bûcher de Peyre Plantade, deux exemplaires associés à une grande pièce de bois avec encoches dans la fosse $4 \mathrm{du}$ Pont de Pierre (Alfonso, 2002, p. 798), deux autres dans la fosse 68 des Communaux de Saint-Cézaire à Vernègues (Chapon et al., 2004, p. 133) et un dans le dépôt de crémation 5050 des Girardes à Lapalud, dans le Vaucluse $(i d$. , ibid.). On note que la plupart de ces décou- 

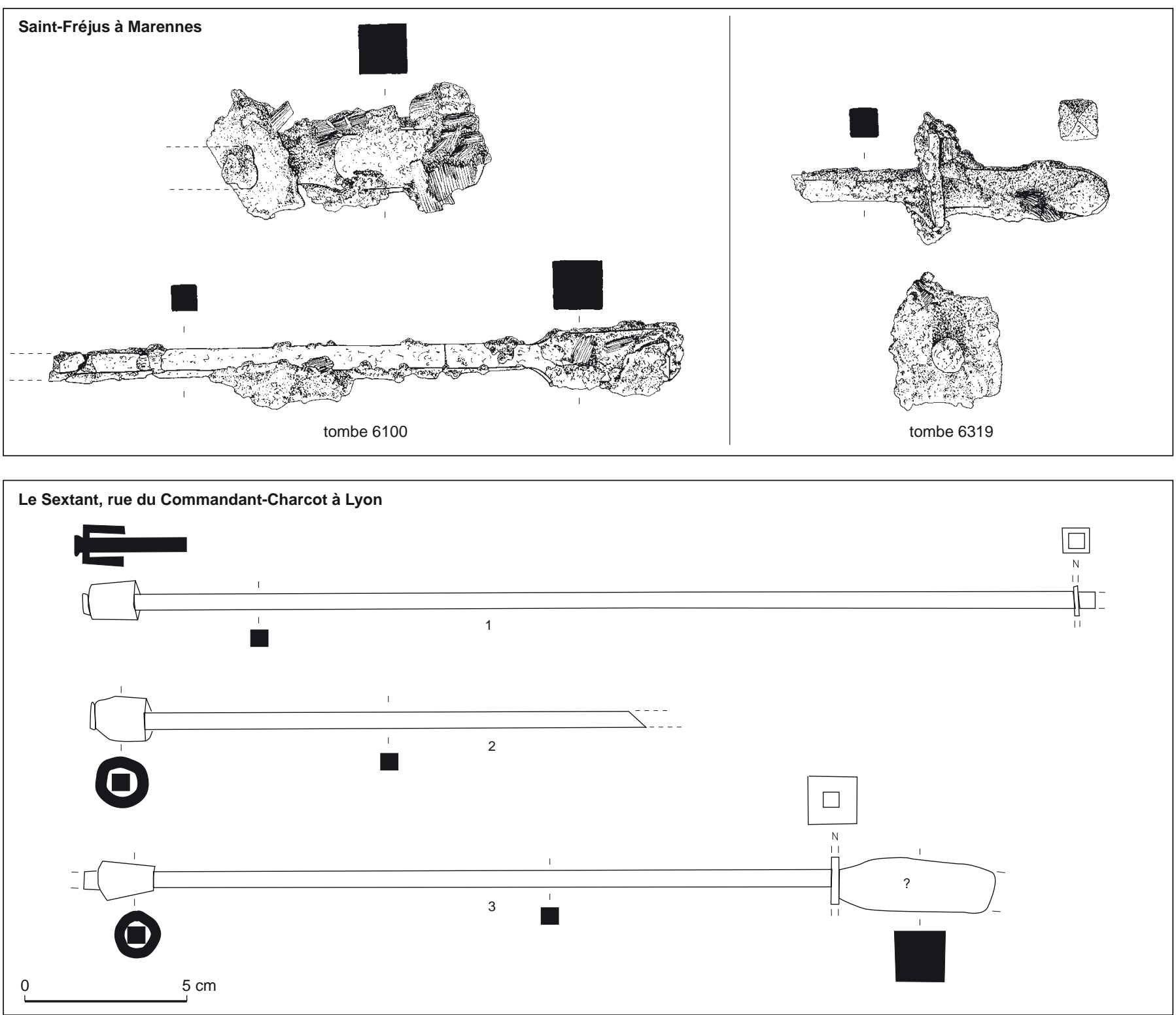

Fig. 94 - Armatures de pieds de lits en fer dans les crémations 6100 et 6319 de Saint-Fréjus à Marennes, Rhône (dessin : M.-N. Baudrand; DAO : G. Macabéo, Inrap) et dans les bûchers 4 et 5 du Sextant, rue du Commandant-Charcot à Lyon (dessin : M.-É. Gagnol ; DAO : G. Macabéo, Inrap).

vertes proviennent de sites ruraux alors qu'en général, les éléments de décor de lit en os sont bien représentés dans les ensembles périurbains. Par ailleurs, les vestiges de lits semblent attestés principalement dans les bûchers de grandes dimensions, peut-être parce qu'ils nécessitaient une plus grande assise.

À Lyon, les éléments de tabletterie se rapportant à des lits funéraires sont rares; seules quelques pièces éparses ont été recensées par J.-C. Béal parmi le mobilier des fouilles de
Trion du XIX ${ }^{\mathrm{e}}$ s. (Béal, 1983 et 1986). Ce constat vaut déjà en soi pour le site de la Favorite, pourtant riche en bûchers de grandes dimensions, puisque aucun bûcher et aucun dépôt de résidus n'en a livré : les quelques fragments de décor de lits proviennent de "fosses dépotoirs " (Tranoy, 1995b, p. 784). Au Valladas, seules 5 à 10 structures en conservent, mais ni sur le site périurbain du Replat à Aime, ni à la Grande Borne à Clermont-Ferrand, aucun élément de ce type n'a été trouvé lors du tamisage des couches de résidus. 
On notera toutefois que dans quelques séries régionales, la tabletterie apparaît dans les inventaires (les Roses, rue du Commandant-Charcot ou Villa Montel à Lyon, la Brunerie à Voiron, par exemple) mais n'a pas été étudiée.

Cependant, des armatures de pieds de lits en fer ont été découvertes sur le site du Sextant, rue du CommandantCharcot, dans quatre bûchers en fosse (fig. 94). Les structures F1 et F4 contiennent une seule tige tandis que les deux autres (F5 et F18) en livrent deux. Ces objets sont constitués de la même manière : une tige en fer de section quadrangulaire $(0,5 \mathrm{~cm}$ de largeur), comportant à une extrémité une virole tandis que l'autre extrémité, plus large souvent brisée et manquante, est précédée d'une plaque de forme carrée. En dépit de la mauvaise conservation du matériau, leur longueur a pu être évaluée à une trentaine de centimètres. Il s'agit de l'unique exemplaire lyonnais connu à ce jour.

La connaissance des modes de construction du bûcher et des dispositifs de présentation du corps et du mobilier repose principalement sur l'analyse de la répartition des artefacts associés aux ossements humains. Ainsi, la distribution par carrés de l'ensemble des éléments de décor de lit en os du bûcher 6200 du site de Saint-Fréjus à Marennes (Rhône) montre une concentration des pièces de placage en os et en ivoire des fulcra et du cadre sur le côté ouest de la fosse, principalement au sud près de la tête (Bel, 1996). Par ailleurs, les éléments de placage en os de pieds de lit sont principalement issus des angles de la fosse, mais la répartition des différents types de décors n'est pas homogène, l'angle sud-ouest livrant des éléments différents. Ces observations font penser que la face décorée du lit était dirigée vers l'ouest, où devait se tenir l'assistance, et que la partie située du côté de la tête présentait une ornementation différente. Dans ce cas, les armatures en fer des pieds de lits se trouvaient dans le comblement de la fosse et ont donc été déplacées après la crémation. La même hypothèse peut être formulée à propos des éléments de tabletterie retrouvés dans la partie nord-ouest du bûcher 225 de Voiron où, d'après la situation des clous de chaussure, les pieds du cadavre étaient supposés se trouver.

L'existence d'un cercueil ou d'un coffre peut être suggérée par la répartition des restes osseux dans la couche de résidus. Toutefois, l'absence de restes dans une partie de la fosse, avec une répartition différentielle des os et du mobilier, conjuguée à des effets de délimitation sur l'ensemble des résidus peut également correspondre à un réaménagement de ces derniers à l'issue de la crémation (voir chapitre III, p. 160-164).
Dans le cas du bûcher de Gallière à Montpellier la préservation de certaines connexions et le maintien global de la cohérence anatomique ne s'accordent cependant pas avec une telle hypothèse. Les restes osseux brûlés sont concentrés majoritairement dans la moitié orientale de la structure, au sein d'une surface de plan rectangulaire (fig. 95). Les éléments des diverses régions anatomiques se distribuent dans l'espace de manière anatomiquement cohérente, avec le crâne au sud (carrés D3/D4, E3/E4), le tronc, les membres supérieurs et les os coxaux au centre du dépôt (carrés D5/ E5, D6) et les membres inférieurs au nord (carrés C7, D7/ E7, D8/E8). La présence des clavicules à l'emplacement des éléments osseux du tronc et des membres supérieurs (carré D5) suggère la préservation de la position anatomique initiale de ces pièces osseuses de la ceinture scapulaire. Cet indice permet de restituer la position du défunt, sur le dos à la fin de la crémation. Certains fragments osseux se trouvent en position marginale, un peu à l'écart de l'ensemble du dépôt, dans la moitié occidentale de la structure. Il s'agit de vestiges du membre supérieur et du tronc (carrés C5/C6), ainsi que de ceux du membre inférieur (carrés B6 et B8). La dislocation des connexions anatomiques affecte l'ensemble du squelette mais la connexion coxo-fémorale est préservée à gauche (carrés C6/D6, D7) .

L'observation in situ des restes brûlés du défunt ne suggère pas de bouleversement ou de dispersion notable dans l'agencement des régions anatomiques. Cet indice, ainsi que celui de la taille des fragments, permettent d'exclure le rassemblement des ossements par des opérations de ratissage ou des manipulations de restructuration dynamique du bûcher funéraire au cours de la crémation ou à son terme.

Par ailleurs, la zone occupée par les restes osseux brûlés est délimitée par des alignements de clous ( 5 clous au sud, 4 à l'ouest et 3 au nord), d'orientation nord-sud et est-ouest, associés à des fragments de bois carbonisés dont un élément d'une longueur de $1 \mathrm{~m}$ et d'une largeur de 0,10 m, conservé contre le bord oriental de la fosse. Une autre série de clous alignés sur un axe est-ouest, au centre de la zone (carrés C5, D5, E5), semble traduire la présence d'un autre assemblage de bois dans le sens transversal. Ces clous ont été retrouvés à des cotes de profondeur différentes, proches de celles des ossements. Leur disposition suggère de restituer un dispositif en bois de plan parallélépipédique, dont seul le montant longitudinal proche de la paroi orientale de la fosse est conservé en état de bois brûlé. Cet assemblage pourrait correspondre à une structure ayant servi de support (de type brancard par exemple) ou de contenant pour le corps 


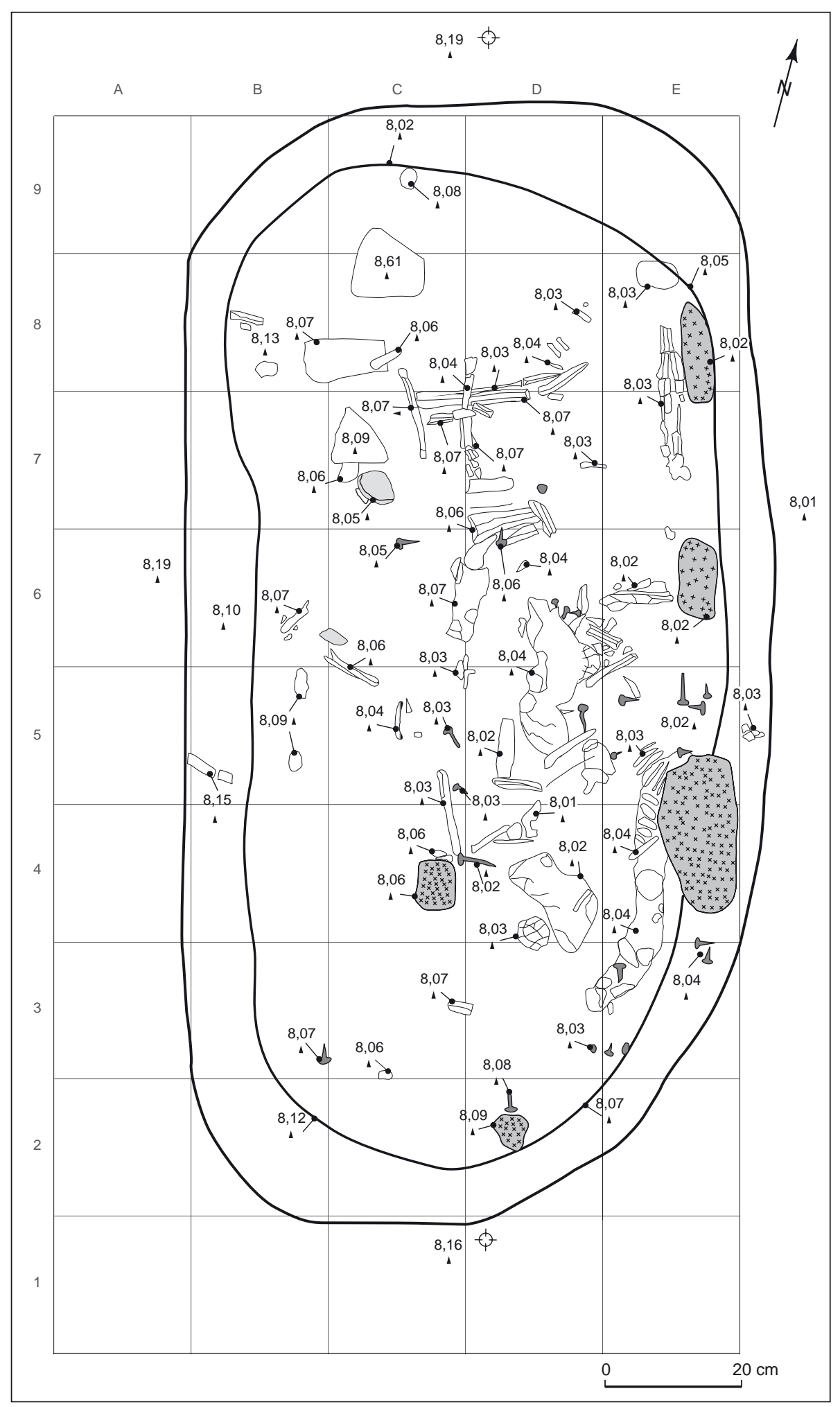

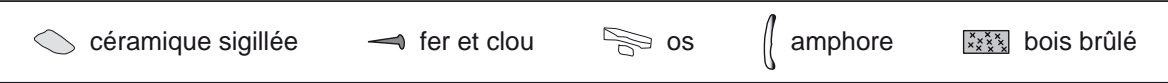

Fig. 95 - La couche de résidus de crémation du bûcher de Gallière à Montpellier, Hérault (relevé et DAO : R. Lisfranc et G. Isnard, Inrap). 


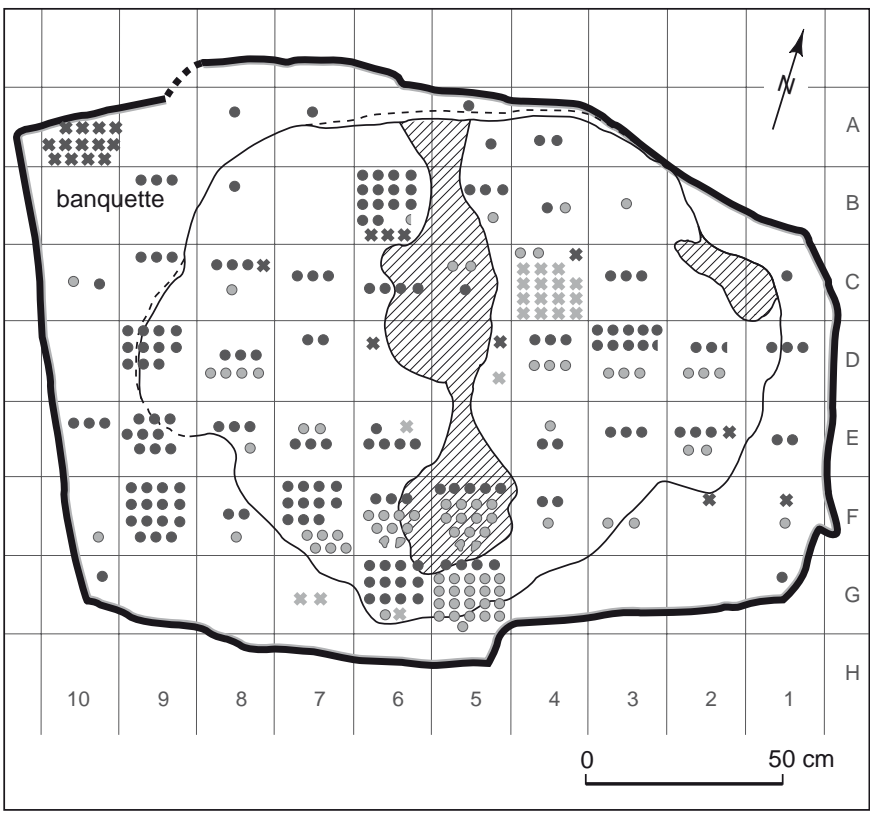

- jeton en os $\quad *$ fragment de jeton en os

- jeton en verre $\quad *$ fragment de jeton en verre $\quad$ ZA zone rubéfiée

Fig. 96 - Répartition des jetons du bûcher 23 de la Raude à Tassin-la-Demi-Lune, Rhône (relevé : J-L. Gisclon, Inrap; DAO : G. Macabéo, Inrap).

du défunt lors de la crémation. Ce dispositif est de dimensions modestes (inférieures à $1 \mathrm{~m}$ de longueur et $30 \mathrm{~cm}$ de largeur). Cependant, cette estimation est fondée sur la disposition des clous et des restes de bois brûlés qui ont subi des modifications importantes pendant la crémation. Ainsi, sa longueur pourrait être supérieure, si l'on suppose la présence de «bras » de brancard qui ne nous seraient pas parvenus.

Certains bûchers et dépôts de résidus livrent des pièces osseuses circulaires, qualifiées, souvent par défaut, de jetons par les auteurs. Ils se présentent en effet comme très proches des éléments utilisés pour le jeu de dames (ou latroncules), mais pouvant aussi être assimilés à d'autres jeux de parcours ou de déplacement. À la lecture des données récentes, ils pourraient avoir servi d'éléments de décor. Dans le bûcher de la Raude, par exemple, ont été retrouvés 171 jetons en os et une centaine en pâte de verre, répartis sur toute la surface (fig. 96). On s'attend en effet à ce que les pièces de jeux soient plutôt rassemblées dans un endroit du bûcher, probablement dans un contenant à l'origine, alors que les éléments de décors, apposés le long des montants du lit ou sur les panneaux du bûcher, vont être retrouvés en grand nombre et répartis sur une grande partie ou toute la surface. Le bûcher 45 du Valladas fournit ainsi un lot de 14 jetons en os, tous groupés dans un angle de la fosse, au sein de la couche de résidus de crémation ; un autre (276) livre également 14 jetons, dispersés dans un tiers de la fosse et associés à des clous décoratifs et des éléments de renforts en fer (Bel et al., 2002, p. 221 et p. 449). Les bûchers 6100 et 6200 de Saint-Fréjus en comptent respectivement 9 et 12, associés à des éléments de lit mais aussi, dans 6200, à un dé à jouer auquel ils étaient associés dans la couche de résidus. Dans le bûcher individuel du Larsou à Réalville, 19 disques en os non percés ont été exhumés. Plats ou bombés, 11 sont décorés sur une seule face, la bombée. Ils étaient dispersés dans la zone charbonneuse, dans la partie sud de la structure de crémation. L'ustion n'a pas été la même pour chacune de ces pièces circulaires (6 ex. ; diam. : $1,40 \mathrm{~cm}$ à $1,70 \mathrm{~cm}$; épaisseur : $0,01 \mathrm{~cm}$ à $0,02 \mathrm{~cm}$ ) ou ovales (8 ex. ; diam. : $1,30 \mathrm{~cm} \times 1,45 \mathrm{~cm} ; 1,30 \mathrm{~cm} \times 1,40 \mathrm{~cm}$ et $1,30 \mathrm{~cm} \times 1,70 \mathrm{~cm}$; épaisseur : $0,01 \mathrm{~cm}$ à $0,02 \mathrm{~cm}$ ). Un jeton incomplet, non percé, brûlé et dont la face bombée est décorée, provient d'une structure incomplète du site de la station FrançoisVerdier et est donc difficilement interprétable.

\section{LE DISPOSITIF DU MOBILIER}

\section{IDENTIFICATION DES VASES EN SITUATION PRIMAIRE}

La plupart des couches de résidus charbonneux des structures de crémation, bûchers et structures secondaires, livrent en quantités variables des vases en céramique, plus ou moins fragmentés et brûlés qui n'en restent pas moins le plus souvent abondants et donnent parfois l'apparence d'un bric-à-brac. L'aspect fonctionnel (vases à liquides, à solides, à parfum, lampes) étant fondamental, on ne peut pour autant occulter les vases en verre, porteurs d'informations, en particulier sur le rôle du parfum dans la cérémonie. Ces vases en céramique et en verre, qui comportent des traces de feu plus ou moins marquées, constituent la plus grande part du mobilier. Parce qu'il est associé à la première étape du rite de la crémation, ce mobilier est qualifié de primaire. Celui-ci se caractérise par une représentativité très variable de ses pièces, allant du vase complet au simple fragment, en passant par des vases brisés à tous les degrés de représentativité. Cette masse de vases entièrement ou partiellement brûlés et fragmentés pose une première série de difficultés lors du tri, de la détermination du nombre de vases et de la représentativité de chacun. Les archéologues confrontés à cette complexité ont appliqué, selon l'état de 
la recherche et le temps qu'ils pouvaient y consacrer, différentes méthodes d'inventaire qui vont du simple comptage des seuls vases bien représentés excluant tous les tessons isolés, jusqu'au catalogue exhaustif, descriptif et interprétatif (dépôt primaire, fragment résiduel...). Cette diversité des choix méthodologiques ne facilite pas les comparaisons d'un ensemble funéraire à l'autre, spécialement pour ce qui concerne - lorsque les remontages le permettent - la question du traitement subi par les vases (bris, mutilations, lacunes), qui bénéficient rarement d'un descriptif exploitable. L'abondance des dépôts primaires de vases, leur fragmentation parfois extrême et la variété des altérations dues au feu sont autant de freins à leur analyse, alors qu'ils représentent pourtant l'essentiel des témoins matériels permettant d'évoquer l'organisation du bûcher (soin du cadavre, repas du défunt), et les rituels se déroulant à proximité du bûcher (banquet des vivants).

Finalement, les questions posées par les vases brûlés et leur classification sont d'ordre interprétatif. Dès les années 1980, des auteurs confrontés à la gestion complexe de cette masse de tessons et aux problèmes de représentativité qu'implique leur calcination (Tranoy, 1995b ; Bel et al., 2002) mesuraient pour la première fois l'étendue des difficultés et tentaient d'y faire face. Seules les céramiques, dont le taux de représentativité semblait correct avec un seuil au-dessus de $20 \%$ à $30 \%$, furent considérées comme des dépôts intentionnels ; parmi eux, les vases identifiés par des tessons brûlés se retrouvèrent classés dans les dépôts primaires et ceux exempts de coups de feu, dans les dépôts secondaires. Il restait donc, aussi bien dans les bûchers que dans les structures secondaires, une quantité plus ou moins importante (de quelques dizaines à plusieurs centaines sur le site de la Favorite) de tessons isolés ou de vases mal représentés, dits « fragments résiduels » généralement interprétés comme ayant été piégés accidentellement dans la structure. Lorsque ces fragments étaient issus de structures secondaires, on supposait généralement qu'il s'agissait d'intrus provenant du bûcher imparfaitement nettoyé entre deux usages. Les auteurs invoquaient également l'introduction, lors des interventions sur le bûcher et du creusement de la structure secondaire, de tessons erratiques issus des recoupements avec d'autres tombes ou des niveaux de surface supposés avoir été jonchés de fragments épars susceptibles de tomber dans la fosse. Dans de nombreux cas, les vases mal représentés même s'ils provenaient avec certitude des niveaux charbonneux de la structure examinée étaient donc exclus des dépôts intentionnels et placés parmi le mobilier résiduel. Cette notion de résidualité, discutée d'ailleurs par les auteurs eux-mêmes, conscients de son inadéquation (Tranoy, 1995b, p. 727), est particulièrement délicate puisque la non-appartenance à la structure étudiée exclut de fait ces vases du cortège du mobilier. En effet, même s'il est raisonnable de considérer qu'une part de ces vases est effectivement intrusive, il est abusif de les classer ainsi $a$ priori et donc de les écarter définitivement de la réflexion. Dans une couche de résidus, chaque fragment de vase brûlé peut être l'ultime témoin, certes bien ténu, d'un dépôt intentionnel, dont la majorité des fragments a été dispersée par les remaniements attestés dans de nombreux bûchers. De plus, dans les structures de dépôts qui conservent les résidus (voir chapitre IV), la quantité ramassée lors de la translation des restes dans la structure définitive influence grandement la représentativité de chaque vase.

Cette exclusion systématique a donc été discutée et remise en cause (Bel et al., 2002, p. 106 ; Blaizot, Bonnet, 2007) pour en arriver à considérer les vases brûlés des couches charbonneuses comme des dépôts primaires possibles, quel que soit leur taux de représentativité. Il s'agit de "dépôts primaires avérés $(\mathrm{P})$ » lorsque ce taux est supérieur à 20-30 \% ou de "dépôts primaires potentiels (P ?) » lorsqu'il est inférieur (Blaizot, Bonnet, 2007). C'est en ce sens qu'il importe de proposer, au moins sur un échantillon pertinent de structures, un catalogue descriptif le plus complet possible de l'ensemble des vases brûlés issus des structures funéraires quel que soit leur état de conservation. Ce catalogue inclut la totalité des vases de chacune des structures, en distinguant clairement les dépôts primaires $(\mathrm{P})$, les dépôts secondaires $(\mathrm{S})$ et les vases ossuaires $(\mathrm{O})$. Cette présentation sous forme de listes en tableau permet de mieux gérer les données et offre une lecture globale et relativement rapide des dépôts de chaque structure qui facilite le travail d'analyse et de comparaison (tabl. XII). Elle apporte de plus une lisibilité correcte de ces données et rend ainsi possible de futures réinterprétations.

Afin d'illustrer de quelle manière il est possible de tenter de nouvelles interprétations grâce à un classement plus descriptif qu'interprétatif du mobilier primaire, nous prendrons l'exemple de l'ensemble funéraire du Replat où les vases brûlés, très fragmentés et rarement intacts, sont, à hauteur des deux tiers, en dessous du taux des $20 \%$ de représentativité (P ?). À l'occasion d'un travail sur le rôle des cruches, donc des pratiques libatoires, lors des funérailles (Blaizot, Bonnet, 2007), nous avons mis en évidence que les structures dépourvues de cruches, qu'elles proviennent du mobilier du bûcher $(\mathrm{P})$ ou du mobilier introduit uniquement dans la structure définitive des restes (mobilier secondaire, S), recélaient systématiquement des fragments de cruche 
Tabl. XII - Tableau synthétique des données céramologiques pour les crémations (représentativité, état et traitement des vases) : exemple du site du Replat à Aime (Savoie).

\begin{tabular}{|c|c|c|c|c|c|c|c|c|c|c|}
\hline Structure & $\begin{array}{l}\mathrm{N}^{\circ} \mathrm{de} \\
\text { vase }\end{array}$ & $\begin{array}{l}\text { Type de } \\
\text { dépôt }\end{array}$ & Brûlé & $\begin{array}{c}\text { Nombre de } \\
\text { fragments }\end{array}$ & $\begin{array}{c}\% \text { de } \\
\text { présence }\end{array}$ & $\begin{array}{c}\text { Partie } \\
\text { absente }\end{array}$ & $\begin{array}{l}\text { Catégorie de } \\
\text { céramique }\end{array}$ & Production & Type & Fonction \\
\hline \multicolumn{11}{|c|}{ Dépôt de résidus 76} \\
\hline & \multirow{3}{*}{1} & \multirow[t]{3}{*}{ primaire } & \multirow[t]{3}{*}{$x$} & $1:$ us 78 & \multirow[t]{3}{*}{$70 \%$} & \multirow[t]{3}{*}{ bord, panse } & \multirow{3}{*}{$\begin{array}{l}\text { céramique } \\
\text { commune }\end{array}$} & \multirow[t]{3}{*}{ siliceuse } & \multirow[t]{3}{*}{ petite jatte } & \multirow[t]{3}{*}{ solide } \\
\hline & & & & $5:$ us 83 & & & & & & \\
\hline & & & & $8:$ us 87 & & & & & & \\
\hline & \multirow{2}{*}{2} & \multirow[t]{2}{*}{ primaire } & \multirow[t]{2}{*}{$x$} & $4:$ us 83 & \multirow[t]{2}{*}{$70 \%$} & \multirow{2}{*}{$\begin{array}{l}\text { bord, panse, } \\
\text { fond }\end{array}$} & \multirow{2}{*}{$\begin{array}{l}\text { céramique } \\
\text { commune }\end{array}$} & \multirow[t]{2}{*}{ siliceuse } & \multirow[t]{2}{*}{ petit pot } & \multirow[t]{2}{*}{ indéterminée } \\
\hline & & & & $8:$ us 87 & & & & & & \\
\hline & \multirow{2}{*}{3} & \multirow[t]{2}{*}{ primaire } & \multirow[t]{2}{*}{$x$} & $1:$ us 83 & \multirow[t]{2}{*}{$30 \%$} & \multirow{2}{*}{$\begin{array}{l}\text { médaillon, } \\
\text { bord, fond }\end{array}$} & \multirow[t]{2}{*}{ lampe } & \multirow[t]{2}{*}{ calcaire } & \multirow[t]{2}{*}{ à volutes } & \\
\hline & & & & $5:$ us 87 & & & & & & \\
\hline & 4 & primaire ? & $x$ & $1:$ us 87 & $2 \mathrm{~cm}$, bord & & $\begin{array}{l}\text { céramique } \\
\text { fine }\end{array}$ & $\begin{array}{c}\text { sigillée } \\
\text { sud Gaule }\end{array}$ & Drag. 35/36 & solide \\
\hline & \multirow{2}{*}{5} & \multirow[t]{2}{*}{ primaire? } & \multirow[t]{2}{*}{$\mathrm{x}$} & $1:$ us 83 & \multirow[t]{2}{*}{ indéterminé } & \multirow[t]{2}{*}{ indéterminé } & \multirow{2}{*}{$\begin{array}{l}\text { céramique } \\
\text { commune }\end{array}$} & calcaire & cruche & liquide \\
\hline & & & & $1:$ us 87 & & & & & & \\
\hline & 6 & primaire ? & $x$ & $2:$ us 87 & indéterminé & indéterminé & $\begin{array}{l}\text { céramique } \\
\text { commune }\end{array}$ & calcaire & cruche & liquide \\
\hline & 7 & & $x$ & $1:$ us 78 & $\begin{array}{c}\text { moins de } 2 \mathrm{~cm} \text {, } \\
\text { panse }\end{array}$ & & $\begin{array}{l}\text { céramique } \\
\text { commune }\end{array}$ & siliceuse & indéterminé & indéterminée \\
\hline & 8 & secondaire & & $1:$ us 80 & entre 90 et $100 \%$ & éclat de & céramique & calcaire & cruche & liquide \\
\hline & 0 & & - & $44:$ us 83 & & & commune & & & \\
\hline & 9 & primaire? & $x$ & $1:$ us 83 & $\begin{array}{c}\text { moins de } 2 \mathrm{~cm} \text {, } \\
\text { bord }\end{array}$ & & $\begin{array}{l}\text { céramique } \\
\text { commune }\end{array}$ & siliceuse & petit pot & indéterminée \\
\hline Bûcher 165 & & & & & & & & & & \\
\hline & 1 & secondaire & - & 9 : us 166 & $80 \%$ & $\begin{array}{l}\text { bord, panse, } \\
\text { anse }\end{array}$ & $\begin{array}{l}\text { céramique } \\
\text { commune }\end{array}$ & calcaire & $\begin{array}{c}\text { cruche } \\
\text { miniature }\end{array}$ & liquide \\
\hline & 2 & primaire? & $x ?$ & 37 : us 166 & $20 / 30 \%$ & $\begin{array}{l}\text { bord, panse, } \\
\text { anse, fond }\end{array}$ & $\begin{array}{l}\text { céramique } \\
\text { commune }\end{array}$ & calcaire & cruche & liquide \\
\hline & 3 & primaire ? & $x ?$ & $1:$ us 166 & $\begin{array}{c}\text { moins de } 2 \mathrm{~cm} \text {, } \\
\text { bord }\end{array}$ & & $\begin{array}{l}\text { céramique } \\
\text { commune }\end{array}$ & siliceuse & pot & indéterminée \\
\hline & 4 & primaire ? & $x ?$ & $1:$ us 166 & $\begin{array}{l}\text { moins de } 1 \mathrm{~cm} \text {, } \\
\text { panse }\end{array}$ & & $\begin{array}{l}\text { céramique } \\
\text { fine }\end{array}$ & $\begin{array}{c}\text { sigillée } \\
\text { non identifiée }\end{array}$ & & indéterminée \\
\hline
\end{tabular}

classés parmi les dépôts potentiels (P ?). En tenant compte de tous les types de dépôts ( $\mathrm{P}, \mathrm{P}$ ? et $\mathrm{S})$, chaque structure est pourvue d'une cruche, information majeure pour l'examen des pratiques libatoires dans une cérémonie funéraire. Les témoignages de dépôt de vases lacunaires sont d'ailleurs régulièrement attestés à l'âge du Fer avec des cas extrêmes comme celui du dépôt de tesson unique dans les tombes du site de Lamadelaine (Titelberg, Luxembourg) (MetzlerZens et al. dir., 1999, p. 403). Des sépultures de La Tène montrent également des dépôts de simples fragments d'amphores (Poux, 1999, p. 28) et déjà au premier âge du Fer, des dépôts de tessons sont interprétés comme le symbole ou la pars pro toto du vase (Dedet, 1992, p. 161).

Les problèmes d'identification du mobilier primaire ne se limitent malheureusement pas aux vases brûlés et à leur taux de représentativité ; il reste en effet l'écueil du classement et de l'interprétation des tessons isolés non brûlés issus des structures de crémation. Les fragments des vases passés sur le bûcher peuvent être brûlés à des degrés très variables et parfois même, ils sont exempts de coups de feu. Ainsi, une interprétation trop hâtive risquerait de classer ces éléments, apparemment non brûlés, et trop isolés pour appartenir à un dépôt secondaire, en « intrusifs » ou " résiduels », alors qu'ils pourraient être les reliquats de dépôts primaires. Il convient donc, dans un premier temps, de rester sur un plan descriptif. Inventoriés de la même manière que les autres vases, ils ne sont pas négligés, mais il faut admettre que les arguments sont faibles pour les interpréter systématiquement comme des dépôts primaires potentiels.

\section{PRÉSENGE/ABSENGE DES VASES EN SITUATION PRIMAIRE}

Pour brosser un tableau succinct du rôle des vases brûlés dans les bûchers de l'âge du Fer dans les régions considérées, il faut rappeler qu'en Auvergne et en Languedoc, d'où proviennent les données laténiennes, les situations sont très contrastées. En Auvergne, les rares vases recueillis sont issus 
Tabl. XIII - Pourcentage de structures contenant des dépôts primaires de vases dans les bûchers lyonnais des sites principaux.

\begin{tabular}{|c|c|c|c|c|}
\hline Localisation & Phase & $\begin{array}{l}\text { Type de } \\
\text { structure }\end{array}$ & $\begin{array}{l}\text { Nombre de } \\
\text { structure }\end{array}$ & $\begin{array}{l}\% \text { de structure avec } \\
\text { dépôt primaire }\end{array}$ \\
\hline Rue des Granges, Lyon & $40-60$ & bûcher & 4 & 100 \\
\hline Les Roses, rue du Commandant-Charcot, Lyon & - & bûcher & 2 & 100 \\
\hline 62, avenue du Point-du-Jour, Lyon & fin $l^{\text {er }} \mathrm{s}$ - -début $\|^{\mathrm{e}} \mathrm{s}$. & bûcher & 1 & 100 \\
\hline La Raude, Tassin-la-Demi-Lune & $50-100$ & bûcher & 1 & 100 \\
\hline \multirow[t]{4}{*}{ Voie de l'Océan, Lyon } & $50-100$ & bûcher & 1 & 100 \\
\hline & \multirow{2}{*}{$100-175$} & bûcher & 2 & 100 \\
\hline & & bûcher? & 4 & 100 \\
\hline & fin IIe s.-début III s. & bûcher & 1 & 100 \\
\hline \multirow[t]{3}{*}{ 77-79, rue Pierre-Audry, Lyon } & $25-50$ & bûcher & 1 & 100 \\
\hline & $50-100$ & bûcher & 1 & 100 \\
\hline & $\left\|\mathrm{e}^{\mathrm{e}}\right\|^{\mathrm{e}} \mathrm{s}$. & bûcher & 2 & 100 \\
\hline \multirow[t]{2}{*}{ 54, rue Pierre-Audry, Lyon } & fin $\mathrm{I}^{\mathrm{er}} \mathrm{s}$-début II $\mathrm{s}$. & bûcher & 3 & 100 \\
\hline & $\mathrm{II}^{\mathrm{e}} \mathrm{s}$. & bûcher & 1 & 100 \\
\hline \multirow[t]{4}{*}{ Rue de la Favorite, Lyon } & $0-50$ & bûcher & 18 & 95 \\
\hline & $50-100$ & bûcher & 43 & 100 \\
\hline & $100-175$ & bûcher & 11 & 100 \\
\hline & fin $\left\|^{e} s .-1\right\|^{e} s$. & bûcher & 3 & 100 \\
\hline
\end{tabular}

du bûcher, à l'exception d'un exemple dans une des tombes aristocratiques de la Cime des Bruyères à Pulvérières. En Languedoc en revanche, les tombes sont exemptes de vases brûlés, le mobilier n'étant constitué que de dépôts secondaires de vases (Bel et al., 2008). Sur certains sites auvergnats, comme Aulnat (Gandaillat), aucun récipient ne semble avoir été placé sur le bûcher, mais les structures ne conservent pas non plus de dépôts secondaires de vases.

À Lyon et à Saint-Paul-Trois-Châteaux, les dépôts primaires de vases sont présents dès l'époque augustéenne (tabl. XIII). De manière générale au Haut-Empire, la majorité des bûchers de Rhône-Alpes conserve des dépôts primaires de vases. À Lyon, on en compte $95 \%$ à la Favorite, $100 \%$ sur le site des Roses, rue du Commandant-Charcot, $100 \%$ rue des Granges ; au sud de Lyon, $100 \%$ des trois bûchers de Saint-Fréjus ; dans les Alpes, $100 \%$ des structures du Replat ; dans la moyenne vallée du Rhône, 97 \% de celles du Valladas. Même si les données sont peu nombreuses en Auvergne, les dépôts primaires de vases sont systématiques dans la dizaine de bûchers actuellement identifiés.

Dans les structures secondaires qui conservent les résidus, les restes de vases brûlés sont également nombreux : au Valladas, de $82 \%$ à $100 \%$ des structures intactes en renferment ; à Lyon, ce sont $100 \%$ des structures des Roses, rues du Commandant-Charcot et des Granges qui livrent du mobilier primaire alors que seulement $63 \%$ à $70 \%$ des structures secondaires de la Favorite sont concernées. Dans les Alpes du Nord, au Replat, toutes les structures secondaires comprennent des vases brûlés et le même constat est valable en territoire helvète dans l'ensemble funéraire du port d'Avenches (Castella, 1987, p. 28). En Auvergne, $83 \%$ à $94 \%$ des 42 structures ont livré des dépôts primaires de vases et en Languedoc, sur un échantillon de 13 structures complètes, 11 comprennent au moins un dépôt primaire de vase.

Les dépôts primaires de vaisselle apparaissent dans près de la moitié (entre $40 \%$ et $52 \%$ ) des contextes funéraires des régions méditerranéennes, qu'ils soient provençaux ou languedociens. En Languedoc, où les petits ensembles sont majoritaires, les plus gros effectifs représentés par les sites du 78 avenue Jean-Jaurès à Nîmes, de Soumaltre et de l'Estel, sont eux-mêmes limités à une vingtaine de structures. Seulement la moitié des six bûchers complets de l'Estel ont livré des dépôts primaires de vases alors que les trois bûchers complets du 78 avenue Jean-Jaurès et les trois bûchers complets de Soumaltre en comprennent tous. Si l'on prend en compte le corpus global de la région, bûchers et structures de dépôts confondus, qui compte 69 structures présentant un bon état de conservation, les vases brûlés apparaissent dans près de $52 \%$ de l'ensemble.

En somme, des vases ont été brûlés avec le corps dans la grande majorité des cas.

\section{QUANTITÉ DE DÉPÔTS PRIMAIRES}

Dans les grands ensembles funéraires périurbains de Rhône-Alpes (la Favorite et le Valladas), le nombre de vases déposés dans les bûchers pour la première moitié du $\mathrm{I}^{\mathrm{er}} \mathrm{s}$. de notre ère, qui varie de un à plus d'une cinquantaine, 
se situe dans une moyenne de 12/13 objets. Sur l'ensemble augustéen des Roses, rue du Commandant-Charcot, les deux bûchers ont livré une moyenne de dix-huit dépôts primaires.

À partir de l'époque flavienne, on constate une baisse du nombre de vases déposés sur le bûcher, qui passe à des moyennes de 5/6 à la Favorite et $4 / 5$ au Valladas. De même, le nombre maximum de vases chute considérablement avec 10 dépôts primaires par structure au Valladas et 20 à la Favorite. Au II $^{\mathrm{e}}$ s., les valeurs restent semblables à celles de l'époque flavienne à la Favorite ; en revanche, les bûchers contemporains de l'ensemble funéraire de la voie de l'Océan à Lyon comprennent une moyenne de 7 dépôts primaires de vases et un maximum de 16. Dans les Alpes, les bûchers du Replat, datés du $\mathrm{II}^{\mathrm{e}} \mathrm{s}$. et du début du $\mathrm{III}^{\mathrm{e}} \mathrm{s}$, livrent une moyenne de 6 à 7 dépôts primaires de vases selon l'origine des résidus (bûcher in situ ou structure secondaire). L'évolution sur les sites funéraires d'Auvergne reste difficile à apprécier, car à ce jour, un seul cas de bûcher est attesté pour le $\mathrm{I}^{\mathrm{er}}$ s., celui du Pâtural à Clermont-Ferrand qui compte 31 vases. En revanche, les trois bûchers du $\mathrm{II}^{\mathrm{e}} \mathrm{s}$. correctement inventoriés contiennent entre 8 et 22 récipients, soit une moyenne d'environ 15 à 16 vases par structure à la Grande Borne et la Buyre à Chappes. Pour la région, le bûcher du col de Ceyssat, avec ses 282 céramiques, apparaît tout à fait exceptionnel (Trément, Humbert, 2005).

\section{SÉLECTION DES VASES}

L'origine des vases impliqués dans les funérailles est une question complexe puisqu'il existe une multitude de situations selon les contextes géographiques, chronologiques, etc. Cette question concerne en premier lieu le réseau d'approvisionnement en céramique. En effet, hormis les exemples auvergnats de vases miniatures et de vases factices à l'embouchure fermée, et le cas lyonnais de petites coupelles «votives ", la majorité des céramiques utilisées lors des funérailles est sélectionnée parmi les vases du quotidien (Martin-Kilcher, 1976, p. 51 ; Steiner, Menna, 2000, p. 96-97 ; Bel et al., 2002, p. 280). Il s'agit essentiellement de la céramique fine de table, de la céramique commune de stockage comme les cruches et, moins fréquemment, de la céramique culinaire destinée à préparer et à cuire des aliments ; plus rarement se trouvent des amphores (Lemaître, 2003). Les nombreux vases à parfum, le plus souvent en verre et les quelques brûle-parfums en céramique commune sont également semblables à ceux que l'on trouve dans les habitats.
L'état des vases, en particulier dans les structures de crémations, et surtout leur aspect souvent très lacunaire, a incité de nombreux auteurs a y voir systématiquement des objets endommagés dans la cuisine et recyclés pour la cérémonie funéraire, de manière à réaliser un minimum de dépense. Mais certaines études ont mis en évidence que l'état fragmentaire des vases, les bris et les lacunes résultaient plutôt de leur traitement lors des diverses étapes du rituel et non d'une récupération systématique de vases usagés (Blaizot et al., 2001 ; Blaizot, Bonnet, 2007). En effet, si l'utilisation de vases provenant de la maison est régulièrement attestée par des objets comportant d'importantes traces d'usure (Pilet, 1980, p. 140-141 ; Lintz, 1991, p. 88) voire, dans quelques cas, des traces de réparation au plomb (Charmasson, 1968, p. 137), notamment au IV s., la majorité des vases ne semble pas obéir à ces critères et plusieurs éléments attestent qu'une partie des vases est destinée spécialement aux funérailles.

Les travaux effectués sur de grandes séries de dépôts de vases ont mis l'accent sur l'accumulation de vases appartenant aux mêmes types et sur la notion de service ou d'assemblage cohérent (Bel, 1993, p. 282). Ces associations de types fonctionnels et morphologiques, qui concernent en particulier la céramique sigillée (Tuffreau-Libre, 1992, p. 123), se doublent parfois de séries de vases apparemment neufs estampillés par le même potier appartenant donc à la même production et au même lot (Lintz, 1991, p. 88). Ce premier élément laisse déjà envisager que la famille du défunt achetait à un marchand toute une partie de la vaisselle nécessaire aux funérailles.

Enfin, la découverte dans les tombes de vases usuels mais comportant des défauts de fabrication tels que d'importantes déformations, des fentes ou des coups de feu dus à un mauvais contrôle de la cuisson dans l'atelier, en somme ce que l'on appelle des ratés de cuisson, démontre également que l'on peut se procurer spécialement pour l'occasion, des vases de second choix impropres pour certains à l'usage domestique (Allain et al., 1992, p. 122 ; Jospin, 1998-1999, p. 21 ; Alfonso, Blaizot dir., 2004, p. 166 ; Nin et al., 2006 p. 136-137 ; Blaizot, Bonnet, 2007, p. 221-222). À la Favorite, dans les structures de la première moitié du $\mathrm{I}^{\mathrm{er}}$ s., les vases en imitations de sigillées italiques du service II, de qualité médiocre, avec divers défauts, illustrent l'existence de catégories propres à la sphère funéraire (Tranoy, 1995b, p. 739-748). Il apparaît donc que les vases, loin d'être toujours récupérés au coup par coup dans le contexte domestique, peuvent aussi constituer un marché, même s'il reste difficile d'en mesurer l'importance. 
Un autre argument est développé ici au moyen d'exemples lyonnais mis en évidence pour la première fois par L. Tranoy dans son étude des dépôts de vases en sigillée des structures funéraires de la Favorite (Tranoy, 1995b, p. 748-754). En effet, l'inventaire des récipients laissait apparaître une prédilection pour des céramiques sigillées des ateliers de Gaule centrale, entre les années 50 et 150 apr. J.-C. Les résultats obtenus récemment, à partir de l'étude du mobilier d'une des grandes «fosses dépotoirs » (fosse 241, voir chapitre IV), confirment la tendance observée par L. Tranoy et vont à l'encontre de celles observées sur les sites d'habitats lyonnais : sur ces derniers en effet, les sigillées cuites en mode A de Lezoux prennent une part minime du marché au I ${ }^{\mathrm{er}}$ s.. Il faut attendre le milieu du $\mathrm{II}^{\mathrm{e}}$ s. pour que les ateliers de Gaule centrale dominent les approvisionnements en céramique sigillée (Bonnet et al., 2003). Nous proposons ici d'exposer les résultats de l'étude récente des sigillées de la fosse 241 et de les confronter avec le mobilier des tombes et des bûchers (Tranoy, 1995b, p. 748-754), de manière à amorcer ainsi une étude comparative des fréquences de ces mêmes produits entre les sites funéraires et les sites d'habitat.

La céramique sigillée des $\mathrm{I}^{\mathrm{er}}$ et $\mathrm{II}^{\mathrm{e}} \mathrm{s}$. découverte dans la fosse 241 réunit au total 2151 tessons et provient exclusivement de deux aires géographiques, à savoir le sud et le centre de la Gaule (tabl. XIV). L'examen attentif des caractères techniques et typologiques de ces fabrications, ainsi que l'étude des estampilles de potiers permettent d'identifier avec plus de précision les différentes sources d'importation de ce mobilier. De fait, les résultats de cette analyse mettent en évidence des échanges commerciaux assez peu diversifiés, dans la mesure où ces derniers se sont essentiellement concrétisés avec le principal pôle exportateur de chaque région. Ainsi, les productions de Gaule méridionale relèvent-elles en priorité des officines de la Graufesenque (Millau, Aveyron). Les importations d'ateliers satellites tels que Banassac ou Le Rozier (Lozère) ne sont pas clairement attestées, tandis que les sigillées de Montans (Tarn) paraissent totalement absentes. Pour ce qui concerne la Gaule centrale, le centre de production de Lezoux a joué un rôle de premier plan dans l'approvisionnement du site, et ce durant deux siècles. Certes, au cours de la première moitié du II $^{\mathrm{e}}$ s., des céramiques sigillées des Martres-deVeyre (Puy-de-Dôme) semblent avoir été employées dans le cadre des cérémonies funéraires. De même, à partir des années 140-150, on constate la présence d'importations issues d'officines plus septentrionales, localisées autour de Vichy (Allier). Il n'en reste pas moins vrai que, tout au long du $\mathrm{II}^{\mathrm{e}}$ s., la majeure partie de la vaisselle de table en sigillée utilisée sur place tire son origine des ateliers de Lezoux.

On sait qu'au I ${ }^{\mathrm{er}} \mathrm{s}$. de notre ère, les potiers du centre de la France se sont contentés de fabriquer une sigillée cuite en mode $\mathrm{A}$, à pâte siliceuse et vernis poreux, de moindre qualité que celle proposée dans le même temps par les ateliers du sud. Dès les années 10-20 apr. J.-C., en effet, ceux-ci lancèrent sur le marché des pièces de vaisselle cuites en mode $\mathrm{C}$, à pâte calcaire et vernis grésé, en tout point comparables à leurs prototypes en sigillée italique. Cet avantage technique indéniable constitue sans doute l'une des raisons pour lesquelles les officines de la Graufesenque imposèrent leur suprématie sur tous les marchés du quart nord-ouest de l'Europe, depuis les années 40 jusqu'à l'arrêt de leur diffusion à longue distance, vers 120 apr. J.-C. Or, les comptages effectués sur le mobilier de la fosse 241 témoignent d'une réalité surprenante : sur l'ensemble des tessons datés entre 20 et 120 apr. J.-C., plus de $55 \%$ sont issus de Lezoux. Cette supériorité se vérifie également au niveau des catégories typologiques; hormis les coupes, dont l'effectif total est d'ailleurs trop faible pour être représentatif, les assiettes et les bols en provenance de Lezoux dépassent systématiquement $50 \%$.

D'un point de vue diachronique, il est encore trop tôt pour déterminer si ce phénomène s'est exercé de manière uniforme tout au long de la période, ou s'il correspond à une anomalie limitée dans le temps. D’une part, la datation exacte de certaines formes de la Graufesenque, notamment de celles qui ont connu une longévité très étendue, reste difficile à appréhender à partir de menus fragments ; d'autre part, s'il est plus aisé de dater les productions de Lezoux grâce aux différentes phases technologiques du $\mathrm{I}^{\mathrm{er}}$ s., le fait que ce mobilier soit largement brûlé rend très délicate son approche chronologique. Néanmoins, nous disposons d'ores et déjà de quelques éléments de réflexion. Les productions millavoises d'époque tibérienne, par exemple, sont extrêmement rares : les formes d'assiette Drag. 17a et Drag. 9, de même que les formes de bol Ritt. 5 et Drag. 27a, ne livrent aucun exemplaire. Seuls quelques tessons d'assiette Ritt. 1, de bol Drag. 24/25a et de coupe Drag. 29a se rattachent à cette période. En revanche, les spécimens de la phase 2 de Lezoux, contemporaine du règne de Tibère, s'avèrent nettement plus abondantes. Si l'on considère, par ailleurs, l'intervalle compris entre les années 60 et 120, il apparaît que les services A à F, créés vers $60-70$, sont composés à $75 \%$ par des importations de Lezoux. D'après ces indices, nous pouvons donc suggérer, à titre d'hypothèse, que la préférence accordée aux sigillées 
Tabl. XIV - Productions céramiques relevées dans la fosse 241 du site de la Favorite à Lyon : NR, nombre de restes.

\begin{tabular}{|l|c|c|}
\hline Toutes catégories et périodes & N.R. & \% N.R. \\
\hline Assiettes/plats & 1066 & 49,56 \\
\hline Bols/coupelles & 741 & 34,45 \\
\hline Coupes & 16 & 0,74 \\
\hline Hautes & 14 & 0,65 \\
\hline Indéterminées & 314 & 14,60 \\
\hline Total & $\mathbf{2 1 5 1}$ & $\mathbf{1 0 0}$ \\
\hline
\end{tabular}

\begin{tabular}{|c|c|c|c|c|c|c|c|c|}
\hline & \multicolumn{2}{|c|}{ Sud } & \multicolumn{2}{|c|}{ Centre } & \multicolumn{2}{|c|}{ Indéterminé } & \multirow[b]{2}{*}{ Total N.R. } & \multirow[b]{2}{*}{ Total \% N.R. } \\
\hline Toutes catégories (20-120 apr. J.-C.) & N.R. & \% N.R. & N.R. & \% N.R. & N.R. & \% N.R. & & \\
\hline Assiettes/plats & 432 & 54,61 & 490 & 49,35 & 3 & 100 & 925 & 51,76 \\
\hline Bols/coupelles & 286 & 36,16 & 326 & 32,83 & 0 & 0 & 612 & 34,25 \\
\hline Coupes & 11 & 1,39 & 4 & 0,40 & 0 & 0 & 15 & 0,84 \\
\hline Hautes & 1 & 0,13 & 12 & 1,21 & 0 & 0 & 13 & 0,73 \\
\hline Indéterminées & 61 & 7,71 & 161 & 16,21 & 0 & 0 & 222 & 12,42 \\
\hline \multirow[t]{2}{*}{ Total } & 791 & 100 & 993 & 100 & 3 & 100 & 1787 & 100 \\
\hline & N.R. & \% N.R. & & & & & & \\
\hline Sud & 791 & 44,26 & & & & & & \\
\hline Centre & 993 & 55,57 & & & & & & \\
\hline Indéterminé & 3 & 0,17 & & & & & & \\
\hline Total & 1787 & 100 & & & & & & \\
\hline
\end{tabular}

\begin{tabular}{|c|c|c|c|c|c|c|}
\hline & & & & & \multirow[b]{3}{*}{ Total N.R. } & \multirow[b]{3}{*}{ Total \% N.R. } \\
\hline \multirow{2}{*}{ Toutes catégories (60-120 apr. J.-C.) } & \multicolumn{2}{|c|}{ Sud } & \multicolumn{2}{|c|}{ Centre } & & \\
\hline & N.R. & \% N.R. & N.R. & \% N.R. & & \\
\hline Assiettes/plats & 19 & 38,78 & 104 & 45,41 & 123 & 44,24 \\
\hline Bols/coupelles & 20 & 40,82 & 58 & 25,33 & 78 & 28,06 \\
\hline Coupes & 2 & 4,08 & 2 & 0,87 & 4 & 1,44 \\
\hline Hautes & 1 & 2,04 & 1 & 0,44 & 2 & 0,72 \\
\hline Indéterminées & 7 & 14,29 & 64 & 27,95 & 71 & 25,54 \\
\hline \multirow[t]{2}{*}{ Total } & 49 & 100 & 229 & 100 & 278 & 100 \\
\hline & N.R. & \% N.R. & & & & \\
\hline Sud & 49 & 17,63 & & & & \\
\hline Centre & 229 & 82,37 & & & & \\
\hline Total & 278 & 100 & & & & \\
\hline
\end{tabular}

\begin{tabular}{|l|c|c|}
\cline { 2 - 3 } \multicolumn{1}{c|}{} & \multicolumn{2}{c|}{ Centre } \\
\hline Toutes catégories (120-200 apr. J.-C.) & N.R. & \% N.R. \\
\hline Assiettes/plats & 100 & 38,76 \\
\hline Bols/coupelles & 99 & 38,37 \\
\hline Coupes & 1 & 0,39 \\
\hline Hautes & 1 & 0,39 \\
\hline Indéterminées & 57 & 22,09 \\
\hline Total & $\mathbf{2 5 8}$ & $\mathbf{1 0 0}$ \\
\hline
\end{tabular}

de Lezoux ne relève pas d'une initiative particulière et ponctuelle, mais qu'elle répond davantage à un choix rationnel et collectif, inscrit dans la durée.

Les quantités et les pourcentages obtenus à partir d'un comptage en nombre de restes prêtent évidemment à discussion, en raison même du caractère aléatoire de cette méthode de quantification qui ne permet pas d'approcher le nombre réel des vases. Pour l'heure, donc, nous ne pouvons garantir que le nombre d'individus en provenance de Lezoux ait été effectivement supérieur à celui des importations de la Graufesenque. Force est d'admettre, cependant, que les comptages en NR ne dénotent quasiment jamais un tel phénomène sur les sites de consommation domestiques ou cultuels. Bien au contraire, les recherches récentes démon- 
Tabl. XV - Origine des céramiques sigillées des structures de crémation des sites funéraires du $5^{e}$ arrondissement de Lyon (30 à 200 apr. J.-C.) : NMI ; nombre minimum d’individus.

\begin{tabular}{|c|c|c|c|c|c|c|}
\hline \multirow{2}{*}{ Sites } & \multirow{2}{*}{ Chronologie } & \multirow{2}{*}{$\begin{array}{c}\text { Total sigillées } \\
\text { NMI }\end{array}$} & \multicolumn{2}{|c|}{ Centre Gaule } & \multicolumn{2}{|c|}{ Sud Gaule } \\
\hline & & & NMI & $\% \mathrm{NMI}$ & NMI & $\% \mathrm{NMI}$ \\
\hline 62 , rue des Granges & $30-60$ & 36 & 29 & & 7 & \\
\hline \multirow[t]{3}{*}{ Rue de la Favorite } & $50-100$ & 215 & 179 & 83 & 36 & 17 \\
\hline & $100-150$ & 42 & 41 & 98 & 1 & 2 \\
\hline & $150-200$ & 6 & 5 vases & & 1 vase & \\
\hline 54-56, rue Pierre-Audry & fin $I^{e r} \mathrm{~s} .-I^{\mathrm{e}} \mathrm{s}$. & 11 & 11 & & 0 & \\
\hline 41, rue Joliot-Curie & fin $I^{e r} s .-\|^{e} s$. & 6 & 6 & & 0 & \\
\hline
\end{tabular}

trent qu'entre le milieu du $\mathrm{I}^{\mathrm{er}}$ s. et le début du $\mathrm{II}^{\mathrm{e}}$ s., les ateliers de la Graufesenque inondèrent de leurs marchandises la plupart des marchés de Gaule intérieure, y compris les débouchés situés sur le bassin de la Loire, qui constitua pourtant l'axe de diffusion privilégié des officines du centre de la France tout au long de leur histoire (Delage, 1998, p. 278-284). Même sur les sites d'habitat arvernes localisés à proximité immédiate des ateliers, comme l'établissement agricole de Beaumont (Alfonso, Blaizot dir., 2004, p. 131), ou encore les agglomérations antiques de Clermont-Ferrand et Vichy, les vestiges de la Graufesenque représentent généralement $75 \%$ à $90 \%$ du mobilier sigillé au cours de la seconde moitié du $\mathrm{I}^{\mathrm{er}} \mathrm{s}$. De la même manière à Lyon, le dépotoir flavien du 117-121 rue Pierre-Audry comprenait $84 \%$ de céramiques sigillées produites dans le sud de la Gaule. Datées de la même période, les levées de terre du fossé de réaménagement de la domus découverte sur le site de la place des Célestins livrent également $89 \%$ de sigillées méridionales.

L'explication de cette tendance particulière tient peut-être au fait qu'à cette époque, l'achat d'une sigillée de Lezoux représentait sans doute un moindre coût par rapport à celui d'une production de la Graufesenque. En effet, la durée de vie des sigillées méridionales devait être plus longue, dans la mesure où le grésage de leur vernis leur assurait une meilleure protection contre l'imprégnation du goût et de l'odeur des aliments. Il est possible, en conséquence, que les participants aux funérailles se soient contentés d'acquérir une vaisselle de qualité inférieure et moins onéreuse, celle-ci étant dans ce contexte, destinée à n'être utilisée qu'une fois pour être rejetée aussitôt. La validité de cette proposition est d'ailleurs étayée par l'aspect général du mobilier, qui paraît globalement neuf. Nous n'avons détecté aucune usure particulière sur les parois des vases, dont l'existence pourrait évoquer un remploi d'objets domestiques. Si l'on compare la sigillée des sites de consommation à celle de la fosse 241, rien n'indique non plus que celle-ci ait consisté préférentiellement en pièces de second choix. Bien que l'exposition au feu et la fragmentation des vases puissent fortement contrarier leur examen, leurs défauts de tournage, de décor ou de finition ne semblent guère plus abondants qu'en milieu domestique. Enfin, la répartition des formes sigillées au sein de l'assemblage offre peu d'originalité par rapport au cortège rencontré sur les sites d'habitat. Si le répertoire de la fosse 241 dévoile un très faible pourcentage de coupes, bien en deçà des valeurs habituellement reconnues, les assiettes et les bols, en revanche, se répartissent grosso modo selon les mêmes types que dans les vaisseliers des habitations (tabl. XIV). Parmi les assiettes, les types Drag. 18-Lez. 58 et Drag. 15/17-Lez. 66 sont largement illustrés durant tout le $\mathrm{I}^{\mathrm{er}}$ s., de même que l'assiette A2-Lez. 15 à partir de l'époque flavienne. Les bols, quant à eux, regroupent principalement des spécimens de type Drag. 27-Lez. 28, Drag. 24/25-Lez. 26, puis A1-Lez. 14 à partir des années 60-70. Tous les autres types apparaissent dans des proportions plus ou moins marginales, relativement conformes aux indices de production que l'on observe dans les ateliers eux-mêmes, notamment à Lezoux.

En somme, tous ces indices laissent supposer que, dans la plupart des cas, la céramique sigillée a fait l'objet d'un achat spécifique au moment des funérailles, auprès de commerçants qui proposaient une marchandise reflétant la mode du moment et destinée indifféremment aux activités domestiques ou funéraires. Dans un souci d'économie, toutefois, le choix des clients a pu se porter sur des fabrications d'une technologie moins aboutie, puisque celles-ci étaient vouées à un usage éphémère. Depuis lors, l'analyse des dépôts de vases d'autres sites funéraires du même secteur de Lyon (62 rue des Granges, 41 rue Joliot-Curie, 54-56 rue PierreAudry...) a confirmé cette tendance (tabl. XV). Au II ${ }^{\mathrm{e}}$ s., la situation s'avère radicalement différente, dans la mesure où les officines du Centre exercent un monopole presque total sur le marché lyonnais. À cette époque, ces ateliers ne fabriquent plus des imitations de céramique sigillée, mais une vaisselle à pâte calcaire et vernis grésé, de qualité égale 
à celle de la Graufesenque au $\mathrm{I}^{\mathrm{er}} \mathrm{s}$. Un tel renversement de tendance rend désormais caduc le gain financier que les céramiques sigillées du Centre pouvaient éventuellement représenter au siècle précédent. Il ne semble pourtant pas que l'on ait cherché à réutiliser des vases usagés ou à acheter des céramiques présentant des défauts de fabrication. Comme par le passé, aucune trace d'utilisation prolongée n'est perceptible sur ce mobilier, qui paraît assez identique à celui des habitats, tant du point de vue de la qualité du façonnage que de la fréquence des types.

\section{RÉPARTITION FONCTIONNELLE DES DÉPÔTS PRIMAIRES}

\section{LE CLASSEMENT FONCTIONNEL}

Le rôle des vases en céramique et en verre recueillis dans les tombes ne peut être abordé sans tenir compte de la fonction initiale des objets. Trois grandes catégories sont représentées : les vases à nourriture solide ou liquide (vaisselle de table, vases pour la cuisine, amphores, stockage), les vases à huiles parfumées et les lampes. La détermination de la fonction d'une partie des vases n'est pas exempte de difficultés et la rareté des témoignages iconographiques laisse peu d'espoir d'obtenir des réponses. Alors que, grâce aux recettes de cuisine, on dispose de descriptifs des vases culinaires, les textes et l'iconographie ne fournissent pas d'informations précises sur l'usage quotidien de la céramique de table : les représentations concernent essentiellement les vases en verre et en métal (Dosi, Schnell, 1986 ; Blanc, Nercessian, 1992). Si une grande part des formes ouvertes en sigillée, plats et coupelles, semble destinée à présenter des mets solides ou des sauces (Polfer, 1996, p. 112 ; Bel et al., 2002), la fonction de certaines formes ne fait pas l'unanimité. En effet, si, par exemple, des formes hautes à parois fines sont manifestement des gobelets, qu'en est-il des petites formes ouvertes dont l'usage peut varier selon les besoins? La question se pose spécialement pour certaines coupelles en sigillée, par exemple, les types Drag. 33, Drag. 24/25, Drag. 27, pour lesquelles, la morphologie ne peut exclure un usage de vases à boire. Par ailleurs, les difficultés du classement fonctionnel varient selon les particularités du répertoire céramique régional (Tuffreau-Libre, 1992 ; Bel, 1993) et l'évolution dans le temps des modes alimentaires (Bats, 1988 ; Mazières, 2002, p. 297).

Des informations sont parfois fournies par la nourriture ou les substances contenues dans les vases déposés dans les tombes (dépôts secondaires de vases ou dépôts d'inhuma- tions). Par exemple, au Valladas, les restes carnés ont généralement été déposés dans des plats en céramique sigillée ; des coupelles contenaient des escargots et une coupe, un œuf. Bien entendu, on ne peut pas généraliser la fonction courante de ces récipients à partir de ces cas, le propos étant plutôt de déposer des nourritures spécifiques au contexte (Scheid, 1984, p. 133 ; André, 2001, p. 215-220), dans des contenants que l'on considère comme appropriés pour l'occasion. Aux difficultés habituelles d'attribution fonctionnelle dans la sphère domestique, s'ajoute le problème de l'utilisation en contexte funéraire qui peut amener à un usage des objets, d'une manière que l'on pourrait qualifier d'opportuniste. Par exemple, on ignore tout de la fonction en tant que dépôt funéraire des vases en céramique commune, en particulier les pots, qui dans la sphère domestique servent à cuire des bouillies de viandes, de légumes ou de céréales. Servent-ils à présenter les mêmes mets lors du rituel funéraire ou sont-ils utilisés à toute autre chose ? Le rôle de vase ossuaire qui leur est fréquemment attribué illustre particulièrement bien ce déplacement de fonction. De la même manière qu'ils peuvent servir occasionnellement à stocker des œufs dans la cuisine comme à Pompéi (îlot I.8), leur usage peut être diversifié lors de la cérémonie funéraire. Par ailleurs, le processus d'inversion entre monde des morts et monde des vivants au sein des pratiques funéraires antiques (Scheid, 1984) peut expliquer le détournement intentionnel de la fonction d'un objet. En somme, exceptés pour les cruches, les gobelets, les plats de service bas et ouverts, le doute subsistera toujours pour une grande part de la vaisselle de table ou culinaire. Au final, d'un commun accord et à défaut de nouvelles données sur le sujet, les archéologues s'entendent généralement sur les fonctions au moins pour la vaisselle de service (Castella, 1987, p. 29 ; Bel et al., 1993, p. 120-121 ; Polfer, 1996, p. 111-112) : plats et coupelles sont associés dans la catégorie des vases à solides, les gobelets et les cruches destinées au service du vin (Desbat, 2003a) sont classés dans les vases à liquides. Les pots et les marmites en céramique commune sont indifféremment classés dans les vases à solides ou dans la catégorie des fonctions indéterminées, les plats en céramique commune dans les vases à solides, et les pichets et les bouilloires dans les vases à liquides.

\section{VASES À NOURRITURE}

À Lyon, les bûchers et les structures secondaires du $\mathrm{I}^{\mathrm{er}} \mathrm{s}$. des ensembles funéraires des rues du CommandantCharcot (les Roses) et des Granges ont d'abord livré une quantité importante de dépôts primaires de vases à parfum 
Tabl. XVI - Répartition des catégories fonctionnelles des céramiques augustéennes sur le site de la rue du Comandant-Charcot à Lyon : BUF, bûcher; MIXTE, dépôt mixte de crémation; OSS, dépôt de crémation en ossuaire; RES, dépôt de résidus ; $C$, céramique ; V, verre.

\begin{tabular}{|c|c|c|c|c|c|c|c|c|c|c|}
\hline Structures & \begin{tabular}{|c|} 
Primaire I \\
solides
\end{tabular} & \begin{tabular}{|c|} 
Primaire I \\
liquides
\end{tabular} & \begin{tabular}{|c|} 
Primaire I \\
Lampe
\end{tabular} & \begin{tabular}{|c|} 
Primaire $I$ \\
parfum
\end{tabular} & $\begin{array}{c}\text { Primaire } \\
\text { indéterminés }\end{array}$ & $\begin{array}{c}\text { Secondaire I } \\
\text { solides }\end{array}$ & $\begin{array}{c}\text { Secondaire I } \\
\text { liquides }\end{array}$ & $\begin{array}{c}\text { Secondaire I } \\
\text { lampe }\end{array}$ & $\begin{array}{c}\text { Secondaire I } \\
\text { parfum }\end{array}$ & $\begin{array}{l}\text { Secondaire I } \\
\text { indéterminés }\end{array}$ \\
\hline BUF 1 & 2 & 2 & & $7 \mathrm{C}, 3 \mathrm{~V}$ & $5 \mathrm{~V}$ & & & & $1 \mathrm{~V}$ & $2 \mathrm{~V}$ \\
\hline BUF 25 & 9 & & & $1 \mathrm{C}$ & $2 \mathrm{C}, 5 \mathrm{~V}$ & & 3 & & & $1 \mathrm{~V}$ \\
\hline MIXTE 7 & & & & $2 \mathrm{~V}, 1 \mathrm{C}$ & 1 & & 1 & & & \\
\hline MIXTE 10 & 4 & & & $2 \mathrm{C}, 2 \mathrm{~V}$ & 5 & & 2 & & $1 \mathrm{~V}$ & \\
\hline MIXTE 18 & 3 & 1 & & & & & & & & \\
\hline MIXTE 16 & 2 & & & $2 \mathrm{~V}$ & 1 & & & 1 & $1 \mathrm{~V}$ & \\
\hline MIXTE 39 & & & & 2 & 4 & & 1 & & & \\
\hline MIXTE 30 & 1 & 1 & & $1 \mathrm{C}, 3 \mathrm{~V}$ & $2 \mathrm{C}, 2 \mathrm{~V}$ & & & & & \\
\hline MIXTE 32 & 1 & 1 & & $1 \mathrm{C}$ & $3 \mathrm{~V}$ & & & & $1 \mathrm{~V}$ & $1 \vee ?$ \\
\hline \multicolumn{11}{|l|}{ OSS 21} \\
\hline \multicolumn{11}{|l|}{ OSS 40} \\
\hline RES 4 & 3 & 2 & & $1 \mathrm{C}, 2 / 5 \mathrm{~V}$ & $1 \mathrm{~V}$ & & & & $1 \mathrm{~V}$ & \\
\hline RES 8 & 2 & & 1 & $2 \vee ?$ & & & & & & \\
\hline RES 11 & 4 & & & & $2 \mathrm{~V}$ & & & & $1 \mathrm{~V}$ & \\
\hline RES? 14 & 2 & 2 & & $3 \mathrm{~V}$ & $2 \mathrm{~V}$ & & & & $1 \mathrm{~V}$ & \\
\hline RES? 29 & & & & & $2 \mathrm{C}, 1 \mathrm{~V}$ & & & & & \\
\hline RES 31 & 7 & 3 & & 4 & 1 & & & & & \\
\hline RES 36 & 2 & & & $1 \mathrm{~V}$ & & & & & & \\
\hline RES 38 & & 1 & & & & & & & & \\
\hline
\end{tabular}

(voir infra, p. 137-138), puis pour ce qui concerne les vases à nourriture, une nette majorité de vases à solides pour de rares vases à liquides. À la Favorite, où le parfum est également bien représenté, $72 \%$ des dépôts primaires de vases à nourriture recueillis dans les bûchers de la première moitié du I ${ }^{\mathrm{er}}$ s. sont des vases à solides. Cette disproportion est encore plus flagrante dans la seconde moitié du $\mathrm{I}^{\mathrm{er}} \mathrm{s}$. avec $82 \%$ de vases à solides et au $\mathrm{II}^{\mathrm{e}} \mathrm{s}$. avec $84 \%$. Dans les cinq bûchers datés fin $\mathrm{I}^{\mathrm{er}}-\mathrm{II}^{\mathrm{e}} \mathrm{s}$. du Replat, les vases à solides sont toujours majoritaires mais de manière moins écrasante avec $63 \%$. Les données obtenues en Auvergne paraissent semblables à celles de Rhône-Alpes, tout au moins pour le $\mathrm{I}^{\mathrm{er}}$ s. Sur le site du Pâtural, on constate en effet que $74 \%$ des objets du bûcher correspondent à des vases à nourritures solides, tandis que les structures secondaires en comptent $77 \%$. Bien qu'elle persiste au cours du $\mathrm{II}^{\mathrm{e}}$ s., cette prédominance semble légèrement décroître, dans la mesure où les vases à solides représentent désormais $61 \%$ à $66 \%$ des dépôts primaires sur les sites de Rochefort à Gerzat (Puyde-Dôme), la Buyre et la Grande Borne. En revanche, au Valladas, le schéma précédent ne se confirme pas puisque les quantités de vases à solides et à liquides sont globalement équivalentes (Bel et al., 2002, p. 126). En Languedoc, 17 bûchers ont livré la somme de 46 dépôts primaires de vases à liquides pour 59 vases à solides soit $56 \%$; 13 dépôts mixtes sont composés de $66 \%$ de vases à solides et 10 dépôts de résidus livrent $73 \%$ de vases à solides. Ces grandes lignes ne doivent pas cacher une certaine variabilité régionale voire locale. Une part infime des structures ne comprend en effet aucun vase à solides ou aucun vase à liquides et quelques-unes, même à Lyon, contiennent plus de vases à liquides que de vases à solides. Toutefois, ces discordances peuvent être liées à la part importante des vases de fonction indéterminée ou à la faible masse des résidus de la structure examinée (tabl. XVI).

En somme, dans la région de Lyon, en Limagne et dans les Alpes, les vases qui constituent la vaisselle de table, mets et boissons, forment, en proportion variable, avec toutefois une nette prédilection pour les vases à solides, l'essentiel des objets passés au bûcher. La moyenne vallée du Rhône et le Languedoc montrent des tendances plus contrastées avec globalement un équilibre entre vases à solides et vases à liquides. Il s'agit le plus souvent de céramiques sigillées (plats et coupelles), accompagnées de vases à parois fines (coupelles et gobelets) et de cruches à pâte claire. Les céramiques communes, pots, marmites, plats, mortiers apparaissent rarement à Lyon parmi les dépôts alors que les pots sont nombreux à Aime. De manière générale, les amphores sont rares, aussi bien dans les structures secondaires que dans les bûchers, où seuls quelques fragments isolés et brûlés apparaissent, par exemple rue Pierre-Audry (bûcher 150) et à la Favorite (bûchers 53, 243 et 405). La comparaison 
Tabl. XVII - Inventaire des fragments de verre dans la structure 35 du site de la rue des Granges à Lyon (S. Fontaine).

\begin{tabular}{|c|c|c|c|c|c|c|c|c|c|}
\hline $\begin{array}{c}\text { Numéro } \\
\text { d'inventaire }\end{array}$ & $\begin{array}{l}N^{\circ} \text { de } \\
\text { vase }\end{array}$ & Brûlé & Type de dépôt & $\begin{array}{l}\text { Nombre de } \\
\text { fragments }\end{array}$ & Non attribués & Catégorie & Type & Forme & NMI \\
\hline sans précision & & $x$ & primaire & & 23 & verre & $?$ & $?$ & \\
\hline \multirow[t]{3}{*}{3} & & $x$ & primaire & 12 & & verre & soufflé turquoise & $?$ & 1 \\
\hline & & $x$ & primaire & & 10 & verre & & $?$ & \\
\hline & & $x$ & primaire & & 6 & verre & & & \\
\hline 5 & & $x$ & primaire & 3 & & verre & soufflé cobalt & balsamaire & 1 \\
\hline 6 & & $x$ & primaire & & 1 & verre & & & \\
\hline \multirow[t]{2}{*}{7} & & $x$ & primaire & & 15 & verre & & & \\
\hline & & $x$ & primaire & 1 & & verre & $\begin{array}{l}\text { moulé cobalt } \\
\text { id. } \mathrm{n}^{\circ} 9 \text { objet } 2 / 3\end{array}$ & coupe côt. & \\
\hline \multirow[t]{6}{*}{9} & \multirow{6}{*}{ objet 2/3 } & $x$ & primaire & 4 & & verre & moulé cobalt & coupe côt. & 1 \\
\hline & & $x$ & primaire & 25 & & verre & soufflé cobalt & balsam. ? & 1 \\
\hline & & $x$ & primaire & 1 & & verre & soufflé émeraude & $?$ & 1 \\
\hline & & $x$ & primaire & 6 & & verre & soufflé incolore & balsam. ? & 1 \\
\hline & & $x$ & primaire & 6 & & verre & soufflé ambre & balsam. ? & 1 \\
\hline & & $x$ & primaire & 2 & & verre & incol. inclusion ambre & & \\
\hline 10 & & $x$ & primaire & & 5 & verre & & & \\
\hline \multirow[t]{5}{*}{11} & & $x$ & primaire & 10 & & verre & $\begin{array}{c}\text { soufflé incol. fil ambre } \\
\text { concentrique }\end{array}$ & $\begin{array}{l}\text { balsam. } \\
\text { Is } 10\end{array}$ & 1 \\
\hline & & $x$ & primaire & 2 & & verre & soufflé violet, fil blanc & $?$ & 1 \\
\hline & & $x$ & primaire & 11 & & verre & soufflé cobalt & $?$ & 1 \\
\hline & & $\mathrm{x}$ & primaire & 11 & & verre & soufflé ambre & $?$ & 1 \\
\hline & & $x$ & primaire & & 22 & verre & ambre & & \\
\hline \multirow[t]{6}{*}{12} & & $x$ & primaire & & 31 & verre & cobalt & $?$ & 1 \\
\hline & & $x$ & primaire & 1 & & verre & $\begin{array}{l}\text { violet blanc } \\
\text { à rattacher } n^{\circ} 11\end{array}$ & & \\
\hline & & $x$ & primaire & 23 & & verre & $\begin{array}{c}\text { incol. inclusion ambre } \\
\text { à rattacher } n^{\circ} 11\end{array}$ & & \\
\hline & & $\mathrm{x}$ & primaire & & 15 & verre & ambre & & \\
\hline & & $x$ & primaire & & 3 & verre & incolore & & \\
\hline & & $x$ & primaire & & 1 & verre & émeraude & & \\
\hline
\end{tabular}

avec les vases brûlés issus d’une des rares aires de crémation correctement fouillée en Gaule, à Septfontaines-Deck au Luxembourg, s'avère très intéressante car M. Polfer constate que $77 \%$ des dépôts concernent les nourritures solides et qu'il s'agit majoritairement de céramiques sigillées (Polfer, 1996, tabl. 11). En conclusion, les dépôts primaires de vases à nourriture concernent le plus souvent la vaisselle à présenter les mets (plats, coupelles) et, en quantité plus variable, les vases à servir les boissons (cruches) et à boire (gobelets). Ces vases sont parfois associés à des restes de nourriture.

Les informations fournies par les récipients en verre des dépôts primaires s'avèrent souvent faibles, en raison d'une importante dégradation causée par l'ustion. Une grande partie de ce mobilier s'avère beaucoup trop fragmentée et déformée - quand le verre n'est pas réduit à l'état d'un agrégat en forme de goutte - pour identifier le nombre et les formes des vases. Sans doute en raison de leur petite taille et de leur morphologie très particulière, les balsamaires sont les objets les plus facilement reconnaissables et de ce fait, ils se trouvent surreprésentés dans les inventaires (tabl. XVII). Or, l'identification des récipients en verre peut modifier sensiblement non seulement le nombre d'éléments qui composent la vaisselle, mais également la part et la nature des vases à liquides et à solides. Par exemple, sur le bûcher de la Raude, le nombre de récipients en verre (hormis les balsamaires), est presque équivalent à celui des récipients en céramique (15 dans le premier cas, 16 dans le second). La céramique compte 11 vases à solides ( 5 coupelles, 4 plats, 2 pots) et 4 cruches, le verre comprend 12 vases à solides (9 plats, 3 coupelles) et 4 gobelets. Ainsi, la vaisselle se compose de 31 récipients et, parmi les vases à liquides, 
les cruches sont en céramique et les gobelets en verre. À la Grande Borne, la vaisselle de verre identifiée (A. Moirin, musée archéologique de Bourges, étude inédite) se compose quasi exclusivement de vases destinés au service des liquides, contrairement à la vaisselle en céramique qui privilégie le service des nourritures solides (un gobelet et une forme non identifiée dans le bûcher 6 ; deux formes ouvertes, une bouteille et une carafe dans le bûcher 12 ; un gobelet et une bouteille dans le bûcher 15). Les trois bûchers totalisent 38 vases en céramique contre 7 en verre. Sur le site du Sextant, rue du Commandant-Charcot à Lyon, qui couvre l'ensemble $\mathrm{du} \mathrm{I}^{\mathrm{er}} \mathrm{s}$. de notre ère à partir du règne de Tibère, les objets en verre sont représentés par $12 \%$ de vaisselle, avec une majorité de formes ouvertes (coupes, bols, plats) puisque les vases à liquides, illustrés par des bouteilles ne forment que $13 \%$ de la vaisselle en verre (Robin, 2008). Au Valladas, la vaisselle en verre constitue $22 \%$ des récipients et elle est composée en majeure partie de pots à anse, de gobelets et de cruches, les assiettes et les pots étant représentés en plus faible part (Bel et al., 2002, p. 129). Malheureusement, sur ce site, où les dépôts secondaires de vases sont très nombreux, il n'a pas été établi de distinction, dans l'analyse, en fonction de la situation, primaire ou secondaire, de ces récipients. L'auteur remarque que, jusqu'au milieu du $\mathrm{I}^{\mathrm{er}} \mathrm{s}$., le verre se rapporte essentiellement aux balsamaires, mais qu'à partir de cette date, ses fonctions se diversifient et celui-ci se substitue partiellement à la céramique fine (id., ibid., p. 181).

\section{LES VASES À PARFUM}

À Lyon, c'est dans les structures de crémation de la première moitié du I ${ }^{\mathrm{er}} \mathrm{s}$. que l'on compte le plus de dépôts primaires de balsamaires en céramique et en verre ; ils sont présents dans 17 bûchers sur 18 à la Favorite, avec une moyenne de 7,7 vases (de 1 à 20). Les balsamaires en céramique s'avèrent une particularité de l'époque augustotibérienne, où ils sont associés aux balsamaires en verre aussi bien à Lyon (rue des Granges, les Roses rue du Commandant-Charcot, la Favorite, voie de l'Océan), qu'en Provence (la Gatasse, Saint-Lambert). Au Valladas, les balsamaires sont présents dans 37\%-41 \% des structures et, jusqu'à l'époque claudienne, ils sont majoritairement déposés sur le bûcher (Bel et al., 2002, p. 142-143). Le bûcher du $\mathrm{I}^{\mathrm{er}} \mathrm{s}$. de Pont de Pierre livre un minimum de 13 balsamaires brûlés et celui de la Raude, daté de l'époque claudienne, en comprend au moins 5, également brûlés. En revanche, dans la ZAC Sextius-Mirabeau, à Aix-en-Provence, les restes brûlés de balsamaires concernent seulement 7 structures de crémations sur 37, contrairement à Vernègues où $42 \%$ des bûchers renferment un ou deux balsamaires et au Languedoc où $39 \%$ des structures en sont pourvues.

À partir de l'époque flavienne, globalement, leur fréquence et leur quantité baissent considérablement, aussi bien au Valladas qu'à Fréjus, où les dépôts primaires de parfum se font plutôt après la crémation dans les structures de dépôt. Présents au Valladas dans $75 \%$ des résidus de crémation avant 70, ils apparaissent dans moins de $30 \%$ des cas à partir du dernier tiers du $\mathrm{I}^{\mathrm{er}}$ s. apr. J.-C. (Bel et al., 2002, p. 142). Au troisième tiers du I ${ }^{\mathrm{er}}$ s. et au $\mathrm{II}^{\mathrm{e}}$ s., la moitié des bûchers de la Favorite livrent encore des balsamaires brûlés (ils en livraient $94 \%$ dans la première moitié du $\mathrm{I}^{\mathrm{er}} \mathrm{s}$.).

Le schéma est très différent dans les Alpes. Au Replat, les balsamaires sont rarissimes avec deux vases non brûlés et quelques grammes d'un exemplaire brûlé dans un bûcher, pour un ensemble d'une centaine de structures. À Avenches, l'ensemble funéraire du port ne compte qu'une tombe pourvue de balsamaires apparemment brûlés (Castella, 1987 , p. 31), tandis qu'aucun n'est répertorié dans celui de la porte de l'Ouest (Castella et al., 1998, fig. 20). Enfin, dans les trois ensembles aristocratiques d'En Chaplix, au riche mobilier (vaisselle en bronze, parure, tabletterie, etc.), les balsamaires sont également très peu nombreux (Castella et al., 2002, fig. 41). De la même manière, ce type de récipient se rencontre rarement en Auvergne. Parmi toutes les opérations archéologiques menées durant les quinze dernières années, un seul contenant en céramique, découvert au Pâtural dans une structure secondaire de crémation, est interprété comme tel. Toutefois, quelques pièces en verre très altérées, pouvant se rapporter à un balsamaire, apparaissent sporadiquement dans les diverses séries. On dénombre ainsi un vase en verre sur un total de 222 dépôts primaires au Pâtural, un sur 35 à la Buyre, mais aucun à la Grande Borne où l'étude du verre a été réalisée par A. Moirin, du musée de Bourges. Le musée Bargoin de Clermont-Ferrand conserve cependant un certain nombre de « lacrymatoires/balsamaires » non brûlés des $\mathrm{I}^{\mathrm{er}}{ }^{\mathrm{II}}{ }^{\mathrm{e}} \mathrm{s}$, dont la plupart est d'origine inconnue ; cinq proviennent des sites des Martres-de-Veyre et un de Cébazat (Ruiz, s.d., p. 18-33). Pour l'heure, la fragmentation élevée des vestiges recueillis dans les structures liées à la pratique de la crémation, ainsi que l'absence d'études spécialisées dans ce domaine, ne permettent pas de déterminer leur fonction avec exactitude. Les rares études réalisées sur des exemplaires de la Favorite et du Valladas montrent la présence d'un 
composé gras non alimentaire d'origine végétale pouvant avoir servi à fixer un parfum (Formenti, Ruby, 1987), et dans un cas (au Valladas), une solution très chargée en chlorure de sodium (Bel et al., 2002, p. 141-142). Ce dernier résultat n'est pas sans rappeler les pleureuses : plaçait-on de l'eau salée dans des fioles pour évoquer les larmes ? Un article récent propose d'examiner neuf des balsamaires recueillis sur le site du Sextant, rue du Commandant-Charcot à Lyon (Garnier et al., 2008) ; quatre d'entre eux ont été placés sur le bûcher et les neuf autres constituent des dépôts secondaires. Les résultats s'avèrent étonnants : les balsamaires non brûlés contiennent une association de cire et de cutine végétales, ces dernières provenant de feuilles attribuables à des espèces feuillues. Les auteurs se demandent si ces flacons n'auraient pas contenu des potions composées de décoctions concentrées de végétaux plutôt que des parfums (id., ibid., p. 78). L'un d'entre eux renferme une sorte de goudron de résineux qui a subi un fort chauffage en atmosphère close, rappelant une huile essentielle. Les balsamaires calcinés ne conservent plus, quant à eux, de matière organique et seuls les composés peu volatils originaires de cires sont présents sous forme de traces.

Quoi qu'il en soit, même si ces récipients, ou la majeure partie d'entre eux, ont pu contenir des essences parfumées, leur dépôt sur le bûcher constitue à l'évidence un phénomène marginal, ce qui ne veut pas dire que les «parfums » étaient rares ou absents, mais que les vases s'y référant explicitement sont peu représentés.

La présence de vases à parfum en Gaule antérieurement à l'époque romaine semble exclusivement concerner les tombes grecques de Marseille (Moliner et al., 2003, p. 205). La pratique de la distillation étant inconnue dans l'Antiquité, il ne s'agit pas véritablement de parfums mais d'huiles systématiquement préparées à base d'olives ou d'une autre plante oléagineuse à laquelle on incorpore des substances aromatiques (Foy, Nenna, 2001, p. 149). Si l'usage d'huiles parfumées répandues avant la crémation sur la dépouille, et plus largement sur le bûcher, est abondamment documenté par les sources écrites grecques et romaines (Scheid, 1984, n. 13), celles qui entrent en scène au moment de la crémation pourraient alors se rapporter à des offrandes aux dieux (id., ibid.) puisque, de manière générale, la sphère religieuse fait grand usage des parfums et des encens. Enfin, on pourrait encore envisager que les parfums témoignent d'une fonction symbolique qui installe le mort dans l'incorruptibilité et, de manière plus pragmatique, de l'utilité de masquer les odeurs de chairs calcinées. En tout cas, le grand nombre de dépôts primaires de balsamaires à Lyon, dans le sud de Rhône-Alpes et dans l'aire méditerranéenne, révèle l'importance des rituels d'aspersion de parfum sur le bûcher dans la vallée du Rhône jusqu'à l'époque flavienne.

\section{LES LAMPES}

La lampe constitue un objet inconnu dans les structures funéraires du second âge du Fer en Rhône-Alpes et en Auvergne, tant sur les bûchers que dans les structures de dépôt. Dans le Midi, en revanche, elles apparaissent, certes en faible quantité, dès le $\mathrm{II}^{\mathrm{e}} \mathrm{s}$. av. J.-C., mais ne sont jamais placées sur le bûcher avec le mort : on les trouve uniquement dans les structures de dépôt, jamais brûlées, des deux premiers siècles avant notre ère. Ainsi, cet élément apparaît comme une innovation.

L'ensemble augustéen des Roses, rue du CommandantCharcot recèle un unique dépôt primaire de lampe. Toujours à Lyon, dans les structures contemporaines de la rue des Granges, trois structures sur sept comprennent des lampes (43\%) ainsi que trois sur quatre datées du milieu du $\mathrm{I}^{\mathrm{er}} \mathrm{s}$. ; toutes les lampes sont passées au bûcher à l'exception d'un cas douteux. À la Favorite, les dépôts primaires de lampe apparaissent dès l'époque augusto-tibérienne avec $67 \%$ des bûchers qui en comprennent le plus souvent un exemplaire et plus rarement deux exemplaires. Quatorze structures de dépôt contemporaines des plus anciennes de la Favorite livrent $36 \%$ de lampes passées au bûcher. À l'époque flavienne, $47 \%$ des bûchers contiennent une lampe, qui constitue le plus souvent l'unique dépôt primaire. Ainsi, de manière générale à Lyon, les lampes sont déposées sur le bûcher et il est plus rare qu'elles apparaissent lors de l'étape de l'ensevelissement.

$\mathrm{Au}$ Replat, dans les Alpes, sur un échantillon de dix structures, $70 \%$ comportent une ou deux lampes brûlées ; les dépôts secondaires de lampe sont inconnus.

Dès l'époque augustéenne dans le midi de la Gaule, les dépôts de lampe existent selon un schéma différent. Au Valladas, seulement $11 \%$ des structures liées à la crémation livrent un dépôt primaire de lampe et elles sont en revanche très nombreuses parmi les dépôts secondaires de mobilier. Dans les bûchers de Vernègues, les lampes sont présentes systématiquement et semblent généralement dégradées et avoir souffert du feu. Alors qu'à Aix-en-Provence, parmi les 37 structures liées à la crémation du site, 3 seulement ont livré des restes de lampes brûlées.

En Languedoc, sur 17 bûchers, seuls 4 comprennent une lampe en dépôt primaire et les dépôts secondaires sont encore plus rares. 
L'Auvergne se démarque encore plus de la tendance générale puisque, sur les sites funéraires fouillés aussi bien dans le passé qu'à une date récente, ce type de mobilier ne semble pas avoir constitué un dépôt très répandu. Les quatre bûchers qui ont pu faire l'objet d'une analyse exhaustive au Pâtural, à la Grande Borne et à la Buyre, ne comportaient aucun fragment de lampe. Cinq exemplaires plus ou moins complets figuraient en revanche dans le comblement de trois structures secondaires du II $^{\mathrm{e}}$ s., situées à Rochefort (Gerzat) et aux Quaires (Les Martres-d'Artière, Puy-deDôme). Deux de ces sépultures se rapportent à des enfants décédés en bas âge, et au vu des découvertes anciennes effectuées dans la région (Mondanel, 1982, p. 74-76), il semblerait que le luminaire ait été associé de préférence à une classe d'âge jeune.

\section{LES DÉPÔTS ALIMENTAIRES}

Les autres catégories sont plus diversement documentées selon les sites. On constate surtout un déficit en études de la faune, des restes végétaux et autres macrorestes, ces éléments étant très fréquemment mentionnés mais rarement étudiés.

Les restes animaux brûlés sont globalement peu présents dans les crémations du Midi méditerranéen, avec des différences selon les secteurs : absents des bûchers de Vernègues, on les rencontre dans une crémation sur dix à Saint-Paul-Trois-Châteaux, dans une crémation sur trois à Aix-en-Provence et une sur quatre en Languedoc. Les espèces représentées sont le Porc (Aix-en-Provence, SaintPaul-Trois-Châteaux, Aspiran et Nîmes), le Bœuf (Aix-enProvence), les Caprinés (Mouton ou Chèvre) (Marseille), la Poule (Aix-en-Provence et Aspiran). On note également la présence ponctuelle de restes de poissons (Aix-en-Provence et Nîmes) et de coquillages (Saint-Paul-Trois-Châteaux et Nîmes). Des espèces non alimentaires comme les Équidés (Aix-en-Provence, Saint-Paul-Trois-Châteaux, Marseille) et le Chien (Aix-en-Provence) apparaissent également. Des restes d'oiseaux sont mentionnés à Saint-Paul-TroisChâteaux et à Nîmes. En Auvergne, les vestiges de faune sont en revanche très présents, comme le montrent des séries récentes issues de contextes ruraux du bassin de Clermont-Ferrand, dont plusieurs sont en cours d'étude par P. Caillat (Inrap). La région se caractérise par l'association presque systématique du Porc et de la Poule, le plus souvent représentés pour le premier par les têtes et les membres postérieurs de trois individus jeunes et pour la seconde par le squelette complet de femelles adultes. Dans le cas où une seule espèce est présente, elle se rapporte aux suidés. Les Caprinés ou le Bøeuf sont ponctuellement relevés, tandis que les animaux pouvant être sauvages (lièvre, tourterelle, canard...) restent minoritaires. À noter qu'aux fragments brûlés sont parfois associés (à Saint-Paul-Trois-Châteaux, sur les sites auvergnats et à Aix-en-Provence, Nîmes), au sein des couches de résidus de crémation, des ossements non brûlés dont l'interprétation pose problème : il pourrait s'agir d'éléments résiduels ou intrusifs ou bien de reliefs de repas introduits en fin de crémation ou postérieurement (Bel et al., 2002, p. 130).

En conclusion, le Porc paraît donc être l'espèce la mieux représentée dans toutes les régions. On remarque également que lorsqu'une seule espèce est illustrée, il s'agit généralement du Porc ou de la Poule. Les séries auvergnates se caractérisent par l'association quasi systématique de ces deux derniers animaux, ce qui n'est pas le cas du Midi, comme le montrent par exemple les résultats obtenus à Aix-en-Provence, où la Poule et le Porc ne sont pas mélangés, mais sont associés majoritairement au Poisson et au Bøuf (Nin et al., 2006, p. 132).

Les restes végétaux (graines, fruits carbonisés, préparations alimentaires) sont malheureusement documentés de manière très inégale, même pour les ensembles fouillés récemment. À notre connaissance, depuis les synthèses publiées sur le sujet par P. Marinval et L. Bouby (Marinval, 1993 ; Bouby, Marinval, 2004, p. 83), les données nouvelles sont peu nombreuses pour les régions qui nous occupent. Sur l'ensemble funéraire de Soumaltre (étude de E. Zwierzinski dans Thernot et al., 2004, p. 209-215), sont attestés des fruits charnus (figuier, vigne), des céréales, des lentilles, des cônes de pin et de cyprès et diverses semences sauvages. Par ailleurs, sur ce même site, un dépôt de résidus a livré un fragment de pain carbonisé de même forme que celui, complet, mis au jour dans le bûcher 4 de l'Estel (Thernot et al., 2004, p. 277). Une étude systématique est actuellement conduite en Auvergne par M. Cabanis sur une douzaine de sites funéraires ruraux du bassin de Clermont-Ferrand. Ses premiers résultats montrent la prévalence de l'Orge vêtue et de la Fève, tandis que la Prunelle, la Vesce cultivée, la Noisette, la Lentille et les Céréales sont en bonne place et que du raisin sec a été relevé à la Grande Borne. Ponctuellement, des préparations alimentaires (pain, galettes, desserts lactés) sont présentes. Enfin, la Figue et la Datte sont attestées sur les sites de la Grande Borne et des Martres-d'Artière. À noter que sur le site des Martres-de-Veyre, des noix, du Raisin frais, des pommes et des poires ont été identifiés dans les inhumations attribuées 
au Haut-Empire (Audollent, 1923). Plus à l'ouest, dans la Creuse, le site des Sagnes, à Pontarion, se distingue par la diversité des taxons cultivés (Marinval, 2001). Ici, c'est le Pois qui domine, suivi par la Fève au détriment de la Lentille. Le Blé est plus fréquent que l'Orge, et si les fruits méditerranéens sont présents (Figue et Datte), de même que la Pêche et le Raisin, la Cerise et la Prunelle sont très fréquentes. Les fruits secs sont illustrés principalement par la Noisette. À Lyon, les quelques contextes étudiés (la Favorite, rue du Docteur-Horand et deux sites sur la rue Pierre-Audry) livrent quelques fruits d'importation comme les dattes ou les pignons de pin, quasiment aucune céréale, un peu de fruits secs (noix et noisettes) et surtout des fruits frais comme la Figue, le Raisin, la Prune et la Pêche, des légumineuses (Fabacées ; Lentille et Vesce) ainsi que de nombreuses pâtisseries (pains ou gâteaux) notamment à la Favorite (Pradat, 1992 ; Marinval, 1993). Globalement, on remarque que dans toutes les régions, les légumineuses et les céréales côtoient les taxons méditerranéens, à l'exception, pour l'Auvergne et Lyon, de l'Olive. Les sites lyonnais se caractérisent par une grande variabilité des taxons représentés selon les sites, par le faible nombre de légumineuses et une importante sous-représentation des céréales relativement à l'Auvergne et au Midi.

\section{LES OBJETS PERSONNELS}

La pratique qui consiste à brûler le défunt avec ses objets personnels est bien attestée à la fin de l'âge du Fer dans le midi de la Gaule. Son ampleur est toutefois difficile à évaluer dans la mesure où l'on a affaire à des éléments placés dans des structures secondaires de crémation dépourvues de résidus. Dans le cas des objets en fer, seules les analyses métallographiques permettent de mettre en évidence une exposition au feu. Les objets présentant des stigmates visibles à l'œil ne sont pas très nombreux. Ils se composent essentiellement d'armes et d'éléments de parure, mais aussi d'objets de toilette et de pièces de coffret (Bel et al., 2008). Le testament du Lingon témoigne du maintien de cette pratique à l'époque romaine en Gaule (CIL, XIII, 5708 ; Le Bohec dir., 1991). On rencontre une très grande variété d'objets dans les structures (Feugère, 1993 ; Tranoy, 1995b, p. 776-783), parmi lesquels dominent les éléments de parure ou de vêtement, les amulettes et dans une moindre mesure, les objets de toilette, les restes de coffret, les pièces de jeu, les outils ou les instruments. Ces objets sont réalisés en métal, en verre ou en os travaillé. De manière générale, les armes sont absentes, sauf celles qui concernent la chasse. Les données archéologiques montrent toutefois que la fréquence du dépôt d'objets varie selon les régions (voir supra, p. 129), mais globalement le mobilier personnel apparaît plus souvent en dépôt primaire qu'en dépôt secondaire. En Auvergne, les objets personnels sont plus rares, même ceux qui ont trait à la parure (perles, épingles, pendentifs...) qui constituent d'ailleurs le seul mobilier de ce type relevé pour l'instant dans les couches de crémation.

Globalement, les objets personnels restent rares et peu diversifiés ; toutefois, l'étude exhaustive des objets menée sur l'ensemble des 19 structures funéraires du site du Sextant, rue du Commandant-Charcot à Lyon (15 bûchers et 4 structures de dépôt couvrant presque tout le $\mathrm{I}^{\mathrm{er}} \mathrm{s}$. de notre ère), a montré que seules 4 structures ne contenaient pas d'objets et que 2 ont révélé des fragments d'objets non identifiables. 6 structures contiennent un seul objet, 3 en renferment 4,4 en ont 2,3 en possèdent 3,1 en livre 4 et enfin 2 en livrent 5 . Le mobilier rassemble donc 34 objets brûlés sur le bûcher (ainsi que 43 clous de chaussure), répartis selon les catégories suivantes : parure (3 structures), accessoires vestimentaires (clous de chaussure : 10 structures; éléments de ceinture : 3 structures), meubles (4 structures), coffrets en bois déterminés par leurs éléments d'assemblages (5 structures), et des objets non classifiés (5 structures). On note également la présence de trois jetons en verre et un jeton en os ne comportant pas de traces de chauffe et issus du comblement supérieur de deux bûchers, sans doute résiduels.

Trois bûchers recèlent des éléments de parure, représentés par des bagues supportant des intailles en pierre ou en pâte de verre. Deux types d'objets se rapportant aux vêtements sont également relevés : des clous de chaussure et des éléments de ceinture en os. Les clous de chaussure sont présents dans 10 structures sur 19. Des éléments de ceintures identiques se retrouvent dans trois structures distinctes où, par ailleurs, le reste du matériel ne présente aucune similitude.

Parmi le matériel métallique, on distingue une série de pièces caractéristiques (éléments de serrure, agrafe, charnières), provenant de petits coffrets en bois. Des éléments identiques ont été recueillis sur le site du Replat, à Aime (fig. 97).

À cela s'ajoute une série d'objets se répartissant dans diverses catégories de mobilier : une applique en bronze, un manche de couteau, une lampe en fer, ainsi que des fragments de silex ne relevant sans doute pas d'un dépôt d'objet stricto sensu mais plutôt d'objets perdus ou abandonnés peut-être lors de l'allumage du bûcher funéraire. 


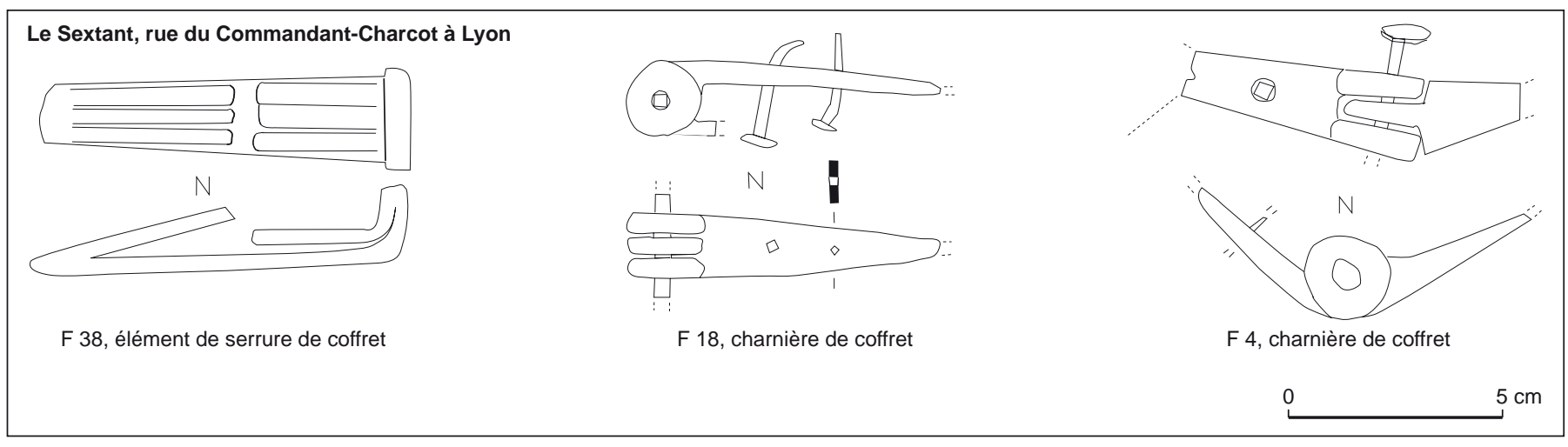

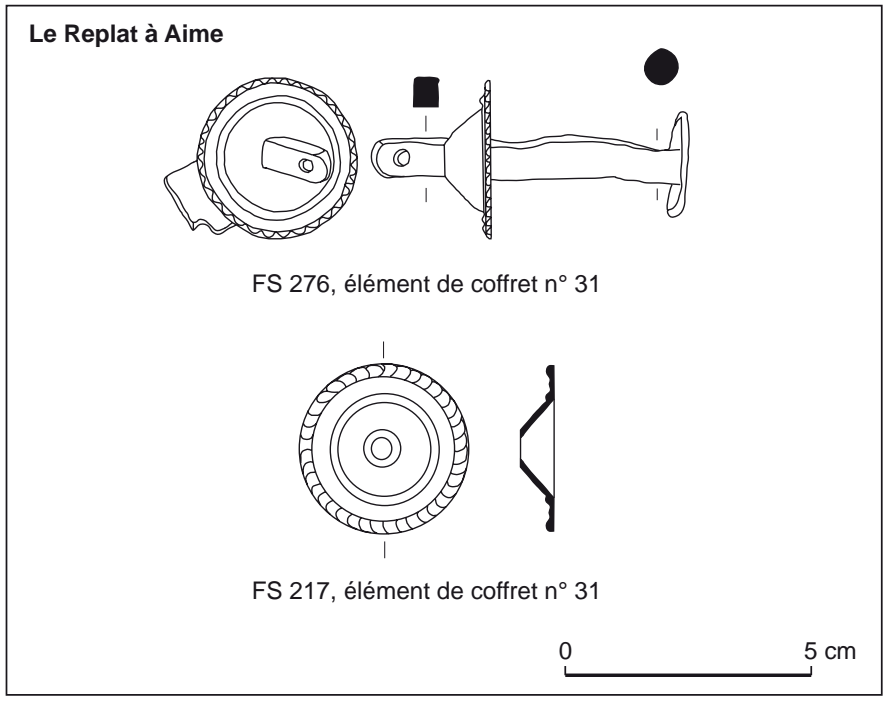

Un bûcher (F47) semble se distinguer des autres par la quantité et la nature des objets qui y furent déposés. En effet, la présence d'un scalpel, d'une ou plusieurs érigne(s) ou aiguilles à cataracte (fig. 98), d'étuis en bronze et d'un minimum de deux coffrets dans la structure indique qu'une trousse d'oculiste fut brûlée avec le défunt. Des forces et une aiguille pourraient avoir complété cet ensemble. Cet ensemble n'est pas la première trousse d'oculiste découverte à Lyon en contexte funéraire ; il s'agit toutefois de l'unique exemplaire connu brûlé sur le bûcher avec le mort : celui de la Favorite, par exemple, a été placé au sommet de la couche de résidus, une fois la crémation achevée. Sur le site du Replat, les coffrets et les accessoires de toilette dominent (fig. 98).

\section{SITUATION DU MOBILIER SUR LE BÛCHER}

Les tentatives de restitution de la disposition du mobilier sur le bûcher se heurtent aux mêmes difficultés que celles qui ont été évoquées précédemment concernant la situation du corps (voir supra, p. 118-121). L'agencement initial est susceptible d'avoir été perturbé par les mouvements internes de la construction liés à la disparition du combustible, mais aussi par les remaniements effectués par les officiants en cours de crémation et à l'issue de celle-ci (voir infra, p. 147-150 et p. 151-164). Par ailleurs, la documentation en ce domaine est tributaire des méthodes d'enregistrement mises en œuvre lors de la fouille. Le nombre de structures pour lesquelles on possède des informations précises et systématiques sur la répartition des restes osseux et sur celle du mobilier est assez réduit. L'effectif se restreint encore si l'on ne prend en compte que les contextes pour lesquels on a pu restituer la position du corps. On ne dispose ainsi au final que de peu contextes utilisables.

La position du mobilier retrouvé dans la couche de résidus de crémation est aussi variable que l'orientation du sujet. La localisation des dépôts dans la zone où se situe la partie inférieure du corps paraît un peu plus fréquente que 



F 47 2/1, fragments d'érigne

F 47 2/1, aiguille

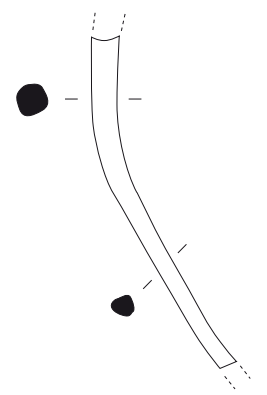

F 47, fragments d'érigne

F 47, fragments d'érigne

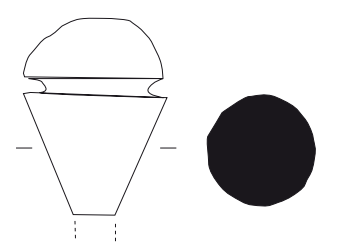

0 $2,5 \mathrm{~cm}$

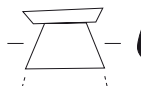

F 47 2/1, fragments d'érigne
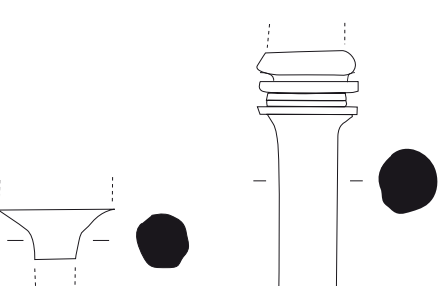

$$
\text { e }
$$




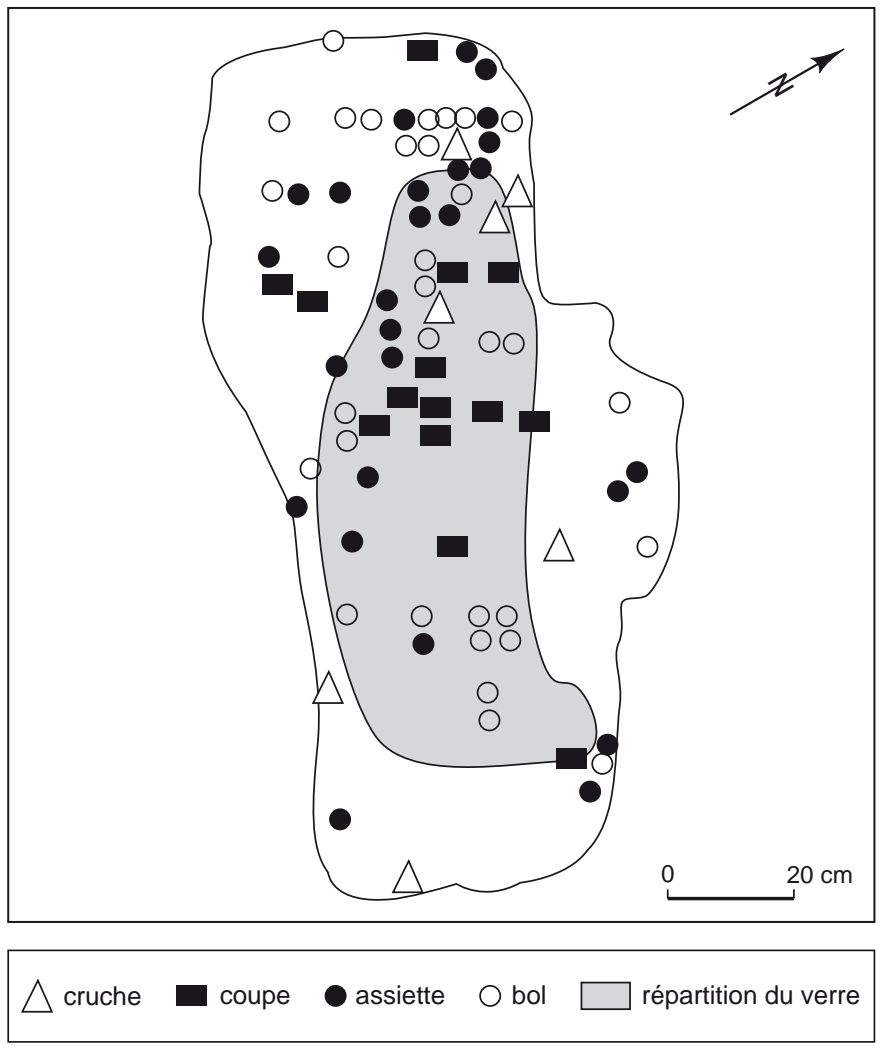

1

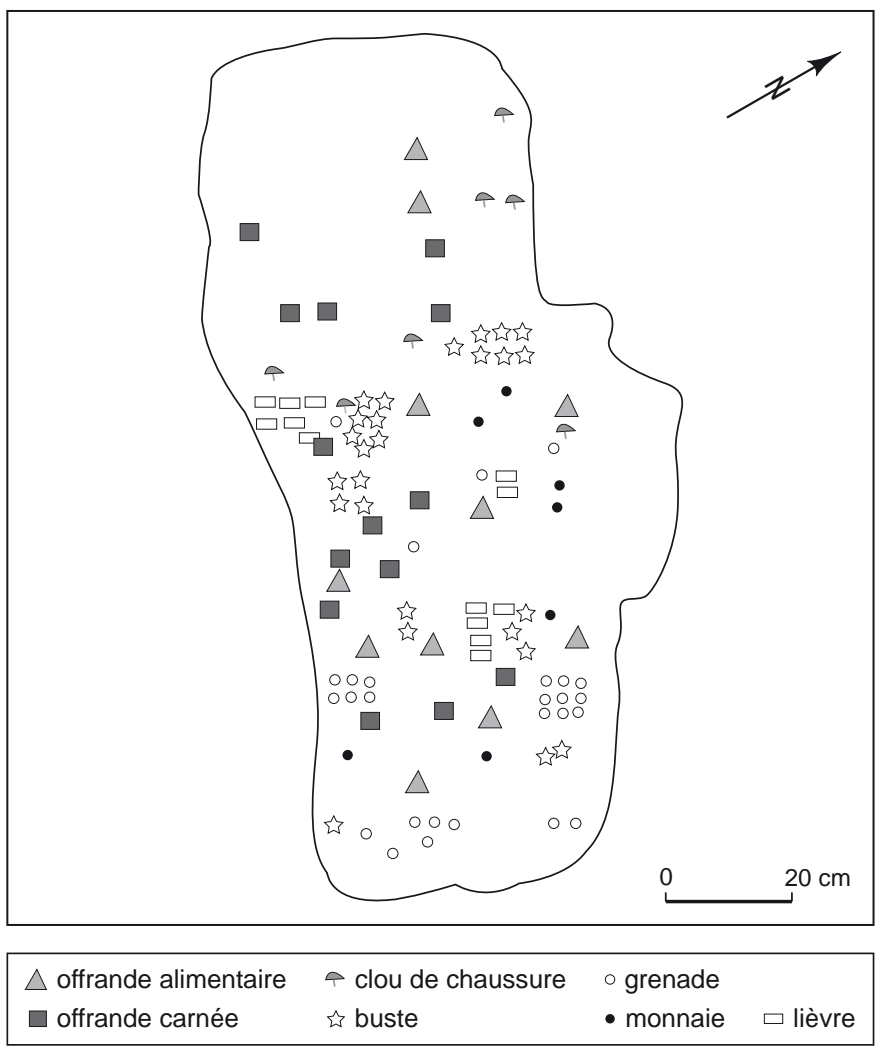

2

Fig. 99 - Le bûcher 25 de la Brunerie à Voiron, Isère : 1, répartition spatiale de la vaisselle; 2, répartition spatiale des objets et dépôts alimentaires (DAO : F. Blaizot, Inrap).

la localisation près de la tête. C'est le cas notamment pour les vases à liquides (sept attestations), les restes de faune (dont la position est documentée dans quatre contextes), les restes végétaux (trois cas : Pont de Pierre, Mas de Vignoles et Gallière) soit en position intermédiaire (deux cas : Sainte-Barbe et Mas de Vignoles). Pour ce qui concerne les vases à solides et les vases à parfum, la localisation près de la tête est presque aussi fréquente que la localisation près des membres inférieurs. Les céramiques du bûcher 225 de la Brunerie à Voiron, se situent majoritairement dans la moitié nord-ouest de la structure, les récipients en verre sont plutôt au centre, et les figurines (lièvre, buste, grenade en terre cuite), les préparations alimentaires et les monnaies sont au centre et au sud-est (fig. 99). Les bûchers auvergnats sont globalement trop remaniés pour livrer de telles informations. Toutefois, dans le bûcher 6 de la Grande Borne, le corps était placé avec la tête au sud, les récipients en verre se trouvaient à l'autre extrémité de la fosse, les restes végétaux au centre, tandis que la céramique et les dépôts carnés étaient répartis sur toute la surface.
Les clous de semelle ont été retrouvés le plus souvent (quatre cas sur cinq) du côté des membres inférieurs. Dans le bûcher 84 du site de Sainte-Barbe (Moliner et al., 2003, p. 292), deux fragments de semelles de chaussure distants de $40 \mathrm{~cm}$ ont été retrouvés, l'un au milieu de la fosse, l'autre près des os du pied gauche en connexion. La couche de résidus de crémation du bûcher 2249 du Mas de Vignoles IX a livré les restes de deux semelles comportant 38 à 41 clous conservés en position fonctionnelle, près du fond de la fosse, dans le même secteur que les os des pieds (fig. 100). On ne peut néanmoins affirmer que les chaussures étaient portées par le défunt dans la mesure où il n'a pas été retrouvé de connexion entre les restes de pieds et les semelles en place. En revanche, dans le bûcher voisin, 2247, la majorité des clous de semelle est issue du secteur où se trouvait la tête du sujet. Dans ce cas, le nombre de clous identifiés ( 8 au total) semble faible pour restituer deux semelles, une paire livrant normalement entre 30 et 130 clous (Van Driel-Murray, 1995). L'éventualité d'un prélèvement d'une partie du dépôt après la crémation n'est 


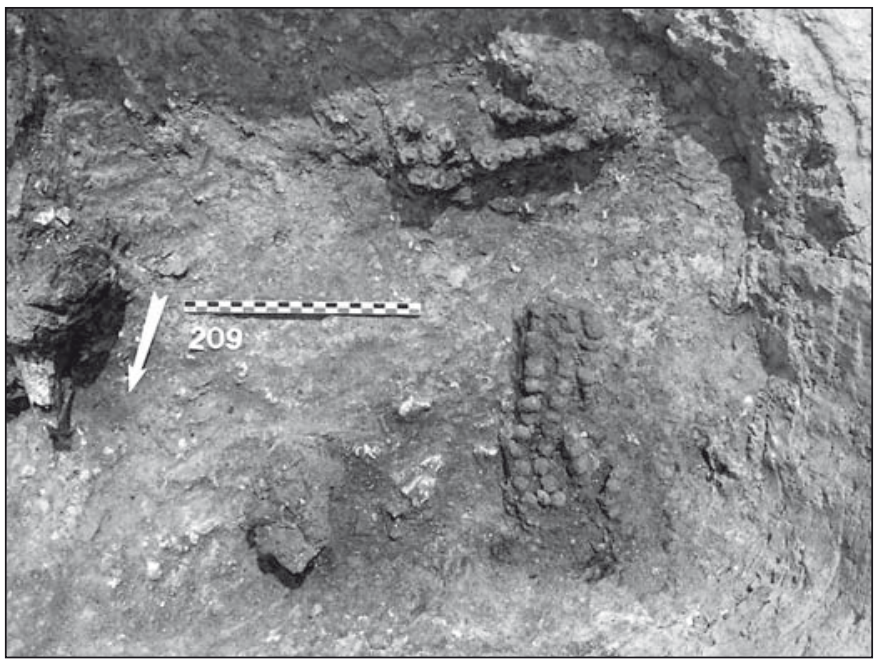

Fig. 100 - Clous de chaussure en place dans le bûcher 2249 du Mas de Vignoles à Nîmes, Gard (cliché : V. Bel, Inrap).

suggérée par aucun indice mais ne peut être cependant exclue.

Dans le bûcher 5 de l'ensemble de l'Estel, orienté nord-est/sud-ouest (fig. 101), presque tout le mobilier brûlé (une coupe, un plat, un gobelet, une lampe et une clefbague) provient du quart sud-est de la tombe, où se situe la partie supérieure du corps d'après la répartition des os du crâne et des membres supérieurs. En revanche, les 146 clous de semelle issus de la couche de résidus étaient localisés à l'extrémité ouest dans le secteur d'où proviennent les os des pieds dont certains étaient en bon rapport anatomique (fig. 91 et 94).

Il existe de nombreux exemples qui permettent de penser que les vases brûlés avec le défunt avaient été placés au-dessous du corps, voire à la base du bûcher, comme au Valladas (Bel et al., 2002, p. 94). Sur ce site, dans 13 cas sur 28, les récipients ont été retrouvés dans la partie inférieure de la couche de cendres, parfois sous les bûches calcinées. Dans le bûcher 5 de l'Estel, contrairement aux ossements qui étaient dispersés sur toute l'épaisseur de la couche de résidus, presque tous les objets brûlés ont été retrouvés entiers et brisés en place sur le fond de la fosse, sauf une coupe (objet 3) qui se trouvait au sommet de la couche charbonneuse. Les pièces de bois carbonisés les recouvraient, à l'exception d'une bûche engagée sous deux récipients (fig. 101). Par ailleurs, tous les vases brûlés ont été retrouvés entiers et brisés sur place, à l'exception d'un gobelet (objet 5), représenté par des éclats caractéristiques d'une fragmentation par la chaleur, dispersés dans un rayon de 0,50 m environ; on peut donc supposer que ces dépôts avaient été pour la plupart placés à la base du bûcher.
Il en est de même dans le bûcher 225 de la Brunerie, où tous les fragments se rapportant au mobilier reposent en grande majorité sur le fond de la fosse, alors que les os humains se situent au-dessus, dans la partie supérieure de la couche de crémation. Il apparaît donc que le mobilier a été disposé par catégories et qu'il se trouvait sous le corps placé, quant lui, au sommet du bûcher.

Dans cinq bûchers de l'Estel, on a retrouvé des éléments de mobilier à la fois sur le fond et au sommet de la couche ; cette configuration indique un agencement complexe ou bien plusieurs moments de dépôts, avant et pendant la crémation. Dans d'autres cas, comme dans près de la moitié des bûchers du Valladas et le bûcher 41 des Communaux de Saint-Cézaire, la situation des vestiges montre que le mobilier était placé le long des parois de la fosse (Bel et al., 2002, p. 94 ; Chapon et al., 2004, p. 172).

Les faits sont cependant difficiles à interpréter dans un grand nombre de cas, comme l'illustre bien, par exemple, le schéma relevé dans le bûcher 1385 aux Luêpes, à SaintPriest. Sur un total de 708 fragments de céramique et de verre, $61,3 \%$ proviennent de la partie supérieure de la couche de crémation ; les ossements humains sont quant à eux, répartis de manière quasi égale de bas en haut (de $21 \%$ à $31 \%$ sur chacun des quatre décapages). Cette répartition signifie-t-elle que le mobilier se trouvait au-dessus du corps ou bien que l'ensemble, corps et vaisselle, reposaient sur un même niveau au sommet du bûcher, mais que les os se sont infiltrés plus largement dans l'épaisseur des résidus?

Certains indices semblent indiquer que quelques vases sont placés en périphérie du bûcher ; il s'agit de vases, brisés ou non, qui comportent de très faibles traces de feu. À la Favorite, certains vases en céramique sigillée ne comportent que de très légères traces de suie ou bien des balsamaires issus des résidus de la crémation ne sont pas altérés, ce qui engendre des problèmes de classification : dépôts primaires ou dépôts secondaires (Tranoy, 1995b, p. 220). À Avenches dans l'ensemble funéraire de la porte de l'Ouest (Castella et al., 1998, p. 183), D. Castella note que «sur nombre d'objets toutefois, les traces de combustion sont très faibles et superficielles, voire absentes ». Cela pourrait signifier que ces vases n'ont pas brûlé au sein du bûcher mais qu'ayant joué un rôle à proximité du bûcher, ils ont après usage été jetés en fin de crémation sur des cendres chaudes. On peut également envisager que certains de ces vases sont tombés sur le fond tout au début de la crémation, là où l'oxygène est déficient et la température très faible. Les expérimentations montrent en effet que les vases déposés dans un bûcher sur lequel il n'y a pas d'interventions de 


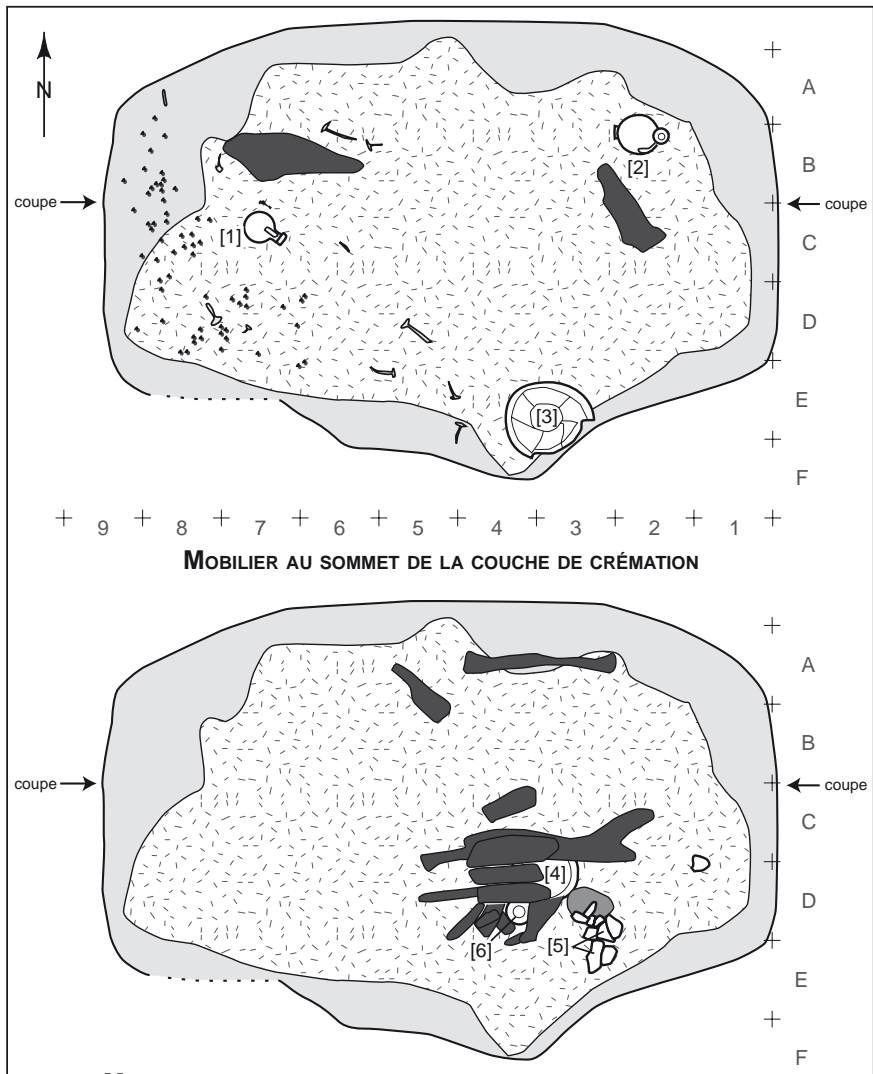

MoBiLIER DANS LA PARTIE INFÉRIEURE DE LA COUCHE DE CRÉMATION

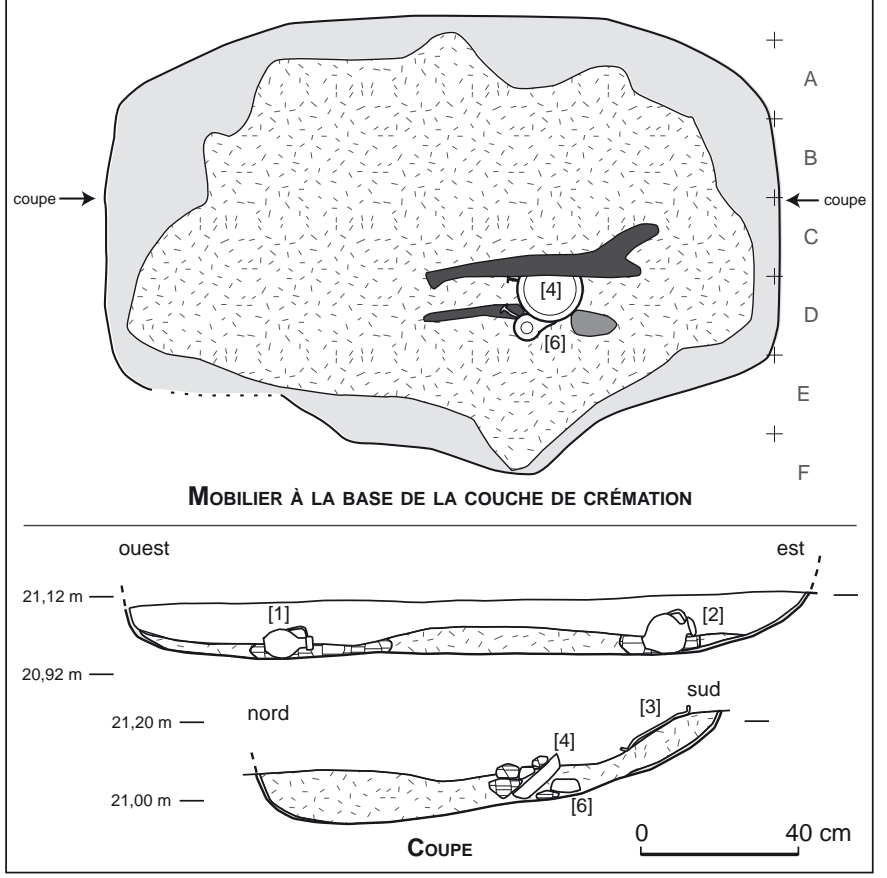

\begin{tabular}{|c|c|c|}
\hline$\Leftrightarrow$ clou & pain carbonisé & zone rubéfiée \\
\hline \# clous de semelle & charbon de bois & zone charbonneuse \\
\hline
\end{tabular}

type conduction peuvent, en chutant, se retrouver hors de son assise et comporter de faibles traces de feu (Courtois, Constantin, 1994, p. 305).

\section{LES BRIS INTENTIONNELS SUR LE BÛCHER}

La notion de représentativité des dépôts primaires de vases (déjà abordée plus haut sous l'angle de la méthode), qui revient à évaluer ce qui reste de l'objet dans la couche de résidus, doit être à nouveau examinée ici. De nombreux récipients extraits des bûchers sont à la fois brisés et lacunaires, même dans les quelques bûchers où la translation des restes n'est pas démontrée. Il est cependant difficile de déterminer si la fragmentation des récipients relève de gestes intentionnels effectués avant ou au moment de leur dépôt sur le bûcher, ou bien de conduite de la crémation (voir infra, p. 147-150), ou encore d'interventions postérieures (voir chapitre III, p. 151-164). Deux hypothèses sont envisageables qui peuvent d'ailleurs être additionnées ; la première propose que les fragments manquants pourraient avoir été projetés à l'extérieur de la fosse car, comme le montrent les travaux ethnologiques (Pautreau, 1994, p. 311 ; Grévin, 2004, p. 49), le travail de l'officiant peut éparpiller les restes sur les niveaux de circulation. La seconde hypothèse est que les prélèvements et autres interventions dans les bûchers ont été plus fréquents qu'on ne le soupçonnait. L'observation des cassures et des traces de feu sur les vases a également permis de mettre en évidence des phénomènes qui pourraient correspondre à différents modes de traitement (fig. 102). En effet, des cassures particulières ont été observées, soit rectilignes et franches qui divisent parfois le vase en deux ou plus (Lintz, 1969 et 1991, p. 89), comme on l'observe au Replat, rue des Granges et à la Favorite, soit sinueuses. Un même vase peut associer les deux types de cassures. Si les cassures sinueuses peuvent correspondre à un choc thermique ou mécanique (effrondrement du bûcher), les rectilignes ne sauraient en résulter ; des expérimentations ont en effet montré que les vases sont très majoritairement retrouvés entiers, rarement brisés par la chute ou les chocs (Courtois, Constantin, 1994, fig. 2). C'est d'ailleurs le cas des récipients issus des bûchers 18 et 22 du site de la rue des Granges à Lyon, qui étaient brûlés, complets et intacts (fig. 103). D’après les expérimentateurs,

Fig. 101 - Répartition des clous de semelle et des restes osseux par régions anatomiques dans le bûcher 5 de l'Estel à Vers-Pont-du-Gard, Gard (relevé : V. Bel, Inrap; DAO : A. Recolin, Inrap). 


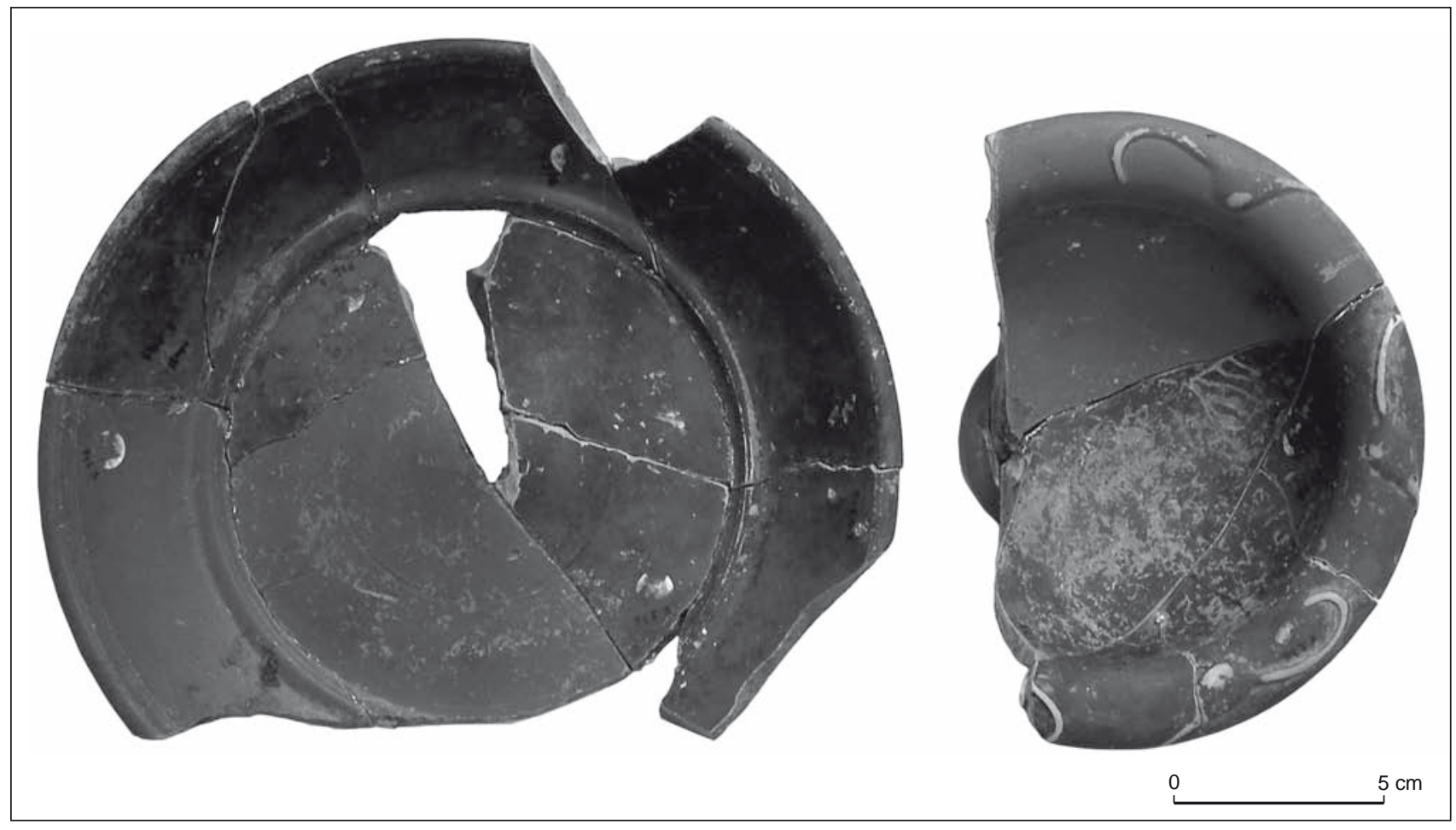

Fig. 102 - Dépôts primaires de vases en céramique sigillée du Replat à Aime, Savoie : cassures rectilignes et ustion différentielle (cliché : J.-M. Degueule, musée de la Civilisation gallo-romaine, Lyon).

un bûcher qui se consume sans intervention ne produit pas de vases brisés, très fragmentés. Ainsi, l'état d'un grand nombre de vases, lacunaires et associant fragments brûlés et non-brûlés sur le même vase, ne correspond donc pas du tout à un scénario statique de crémation mais au contraire à un déroulement très interventionniste.

Les traces de feu observées sur les céramiques sigillées et les collages obtenus entre des tessons très brûlés et d'autres exempts de traces de feu nourrissent encore cette question (Van Doorselaer, 1967). Ces constats ont été effectués sur les vases de la majorité des sites étudiés récemment, aussi bien à Lyon (rue des Granges, la Favorite), que dans les Alpes (le Replat), en Suisse (Martin-Kilcher, 1976 ; Castella, 1987), en Auvergne, ainsi que plus au sud, à Pont de Pierre (Bollène, Vaucluse) et à Mas de Vignoles IX (Nîmes, Gard). Aux Communaux également, les auteurs interprètent la variabilité des traces d'exposition au feu sur les formes complètes comme l'argument d'un bris effectué avant le dépôt dans le bûcher (Chapon et al., 2004, p. 124). Assurément, des vases en sigillée extraits des résidus du bûcher présentent après recollage l'aspect d'une mosaïque de couleur rouge et noire tant le contraste est fort d'un fragment à l'autre. À la Raude, les vases sont très fragmentés et leurs tessons dispersés ; seuls un à deux fragments sur les restes de chaque vase portent des traces de crémation, les autres étant seulement couverts de suie. Il en est de même sur le bûcher de Pont de Pierre, où neuf vases en céramique sigillée, complets et brisés, ne comportent qu'un ou deux fragments brûlés. Or, un scénario dans lequel un important bris de vase aurait projeté certains tessons sur le fond de la fosse où la température est basse, et d'autres du même vase hors du bûcher, paraît très improbable. Les interventions de l'officiant peuvent être invoquées, mais cela impliquerait qu'elles soient relativement brutales pour casser des plats en sigillée relativement solides en deux ou trois fragments, et surtout, elles sont incompatibles avec le fait qu'une partie du vase ne semble jamais avoir approché le feu.

Les bris, les lacunes, l'aspect des cassures et certains des coups de feu différentiels suggèrent plutôt que de nombreux vases ont été brisés avant de passer au feu. Les cassures rectilignes pourraient correspondre à un bris intentionnel où le vase est pris en main et cassé, cette action brutale dispersant les divers fragments dans le bûcher ou hors feu, en brûlant certains et en évacuant d'autres (Dupuy, 1968 ; Perraud, 1974 ; Barthélemy, 1987 ; Dussot, 1987 ; Lintz, 1991, p. 89). Cette situation est en fort contraste avec les cas où les vases 


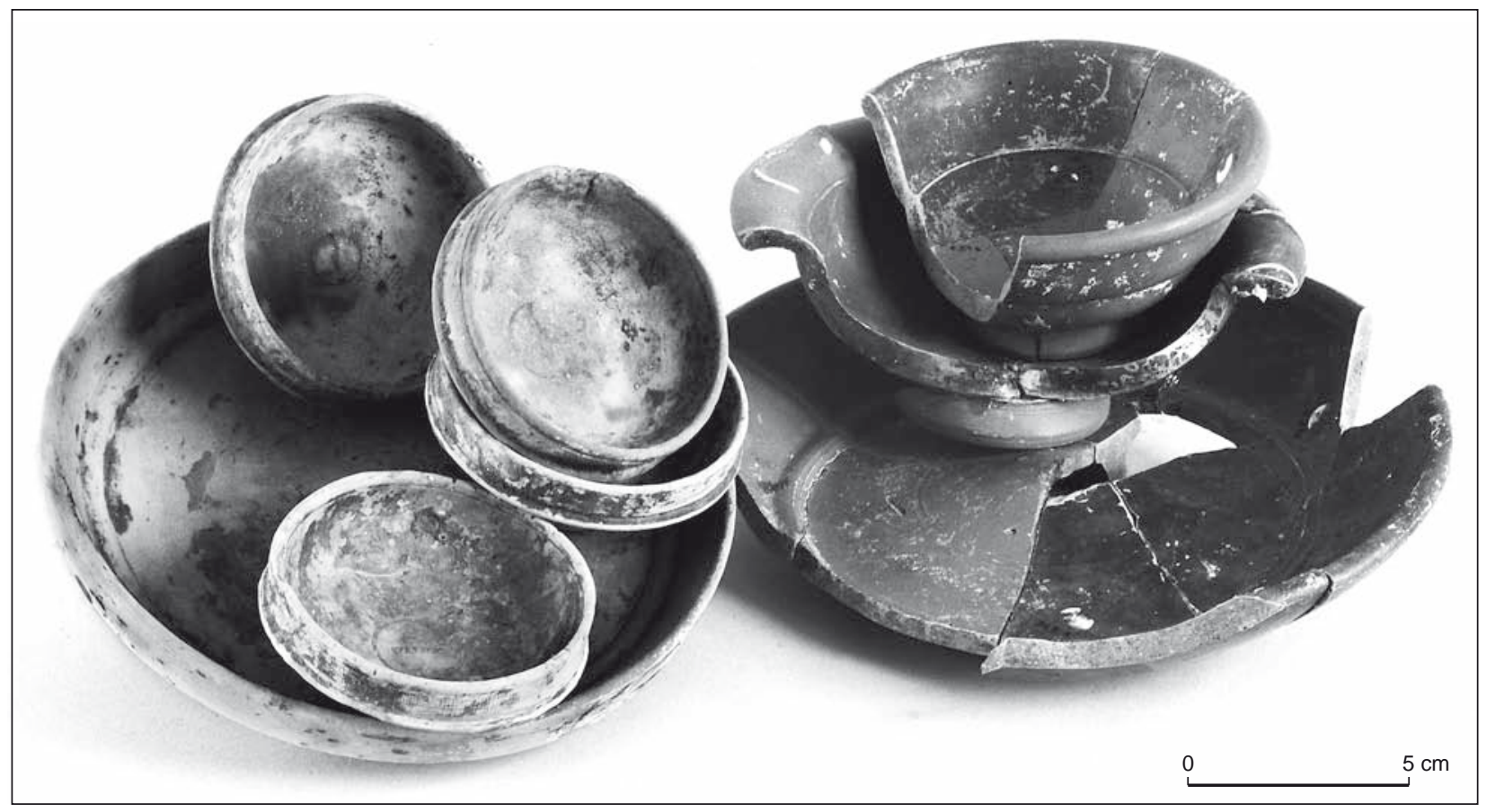

Fig. 103 - Dépôts primaires de vases en céramique sigillée : à gauche, vases brûlés intacts des structures 18 et 22 de la rue des Granges à Lyon; à droite, vases brûlés fragmentés lacunaires de la structure 276 du Replat à Aime, Savoie (cliché :J-M. Degueule, musée de la Civilisation gallo-romaine, Lyon).

retrouvés intacts ont incontestablement été installés dans le bûcher (Bel et al., 2002, p. 94), représentant peut-être le repas du mort alors qu'en opposition, les vases décrits plus haut pourraient correspondre aux vestiges passés au feu d'un véritable repas partagé, pris sur le lieu de la crémation. Dans cette société obsédée par la souillure, les instruments du repas des vivants, pris dans l'aire réservée aux morts, ne peuvent en aucun cas être réintroduits dans la sphère domestique, mais doivent être détruits et brûlés. La destruction de la vaisselle des vivants sur le bûcher du mort rappelle ce qui est observé de nos jours en Thaïlande : les participants aux funérailles consomment des boissons près du bûcher dans des gobelets en plastique qui sont rejetés dans le feu ou éparpillés sur le sol et dont on retrouve même parfois des vestiges jusque dans les vases ossuaires (Pautreau, 1994, p. 313).

\section{LA CONDUITE DE LA GRÉMATION}

Nous avons, à plusieurs reprises, évoqué nos difficultés à restituer l'ensemble du dispositif du bûcher en raison d'un mélange important des artefacts dans la couche de crémation. Ce mélange peut être attribué aux modalités de construction et d'effondrement du bûcher, aux pratiques postcrématoires (voir infra, p. 151-164), mais aussi aux modalités mêmes de la crémation lors du ringardage (ou conduction) qui consiste à activer la combustion en réduisant le bûcher (Pautreau, 1994, fig. 7 ; Grévin, 2004 et 2005).

Le bûcher 225 de la Brunerie est tout particulièrement représentatif d'une telle pratique. Bien que des os soient présents sur toute la surface de la fosse, la masse la plus importante se situe dans la moitié sud-est ; par ailleurs, on n'observe aucune répartition spatiale différenciée des régions anatomiques tandis que les liaisons par collages, réalisées sur les os longs, témoignent d'une forte dispersion des fragments (fig. 104). Seuls les os des pieds associés à la quasi-totalité des clous de chaussure, situés à l'extrémité opposée (nord-ouest), suggèrent que le corps était placé la tête au sud-est et les pieds au nord-ouest. Or, ces données, qui impliquent un rassemblement des restes humains dans une moitié de la fosse, sont en contradiction totale avec la disposition du mobilier, puisque les objets sont encore répartis par catégories distinctes (fig. 99). La concentration de la majorité des os dans une moitié de la fosse, conjuguée au brouillage des différentes régions anatomiques et à la dispersion des fragments osseux se rapporte plus probablement à une conduction du corps, d'autant plus que l'analyse 


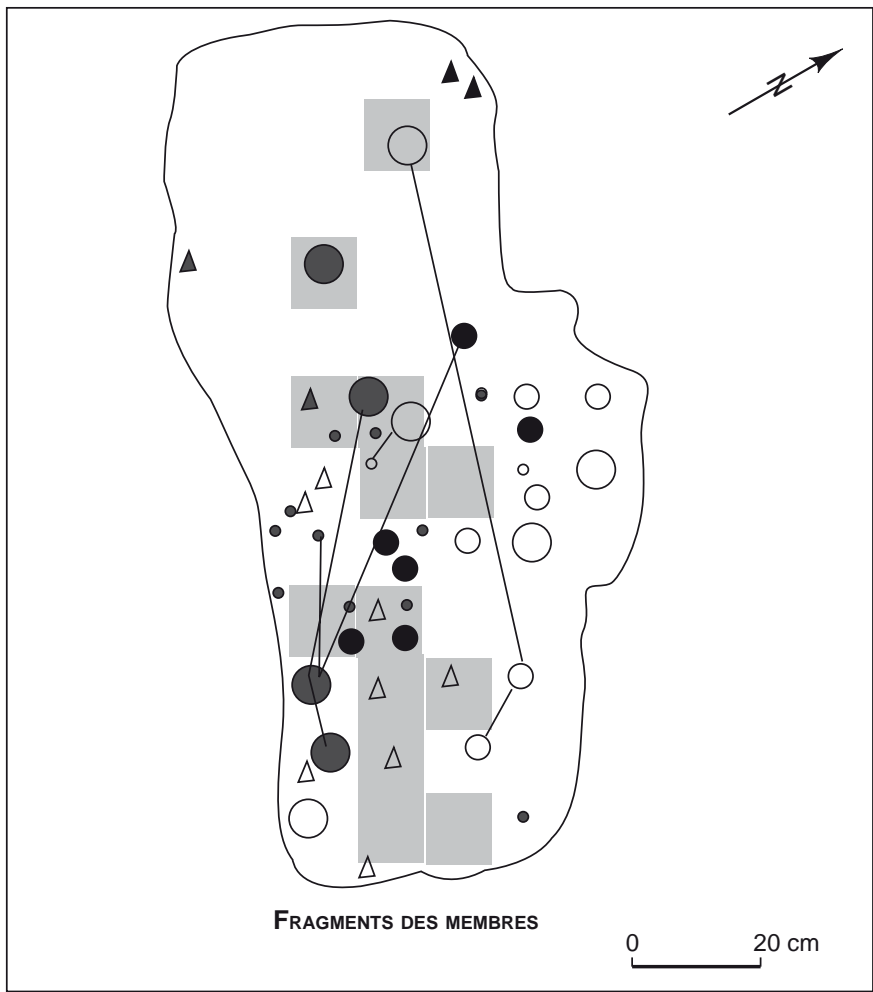

membres inférieurs

membres supérieurs

$\Delta$ pieds

$\Delta$ mains collages

os des membres indéterminés

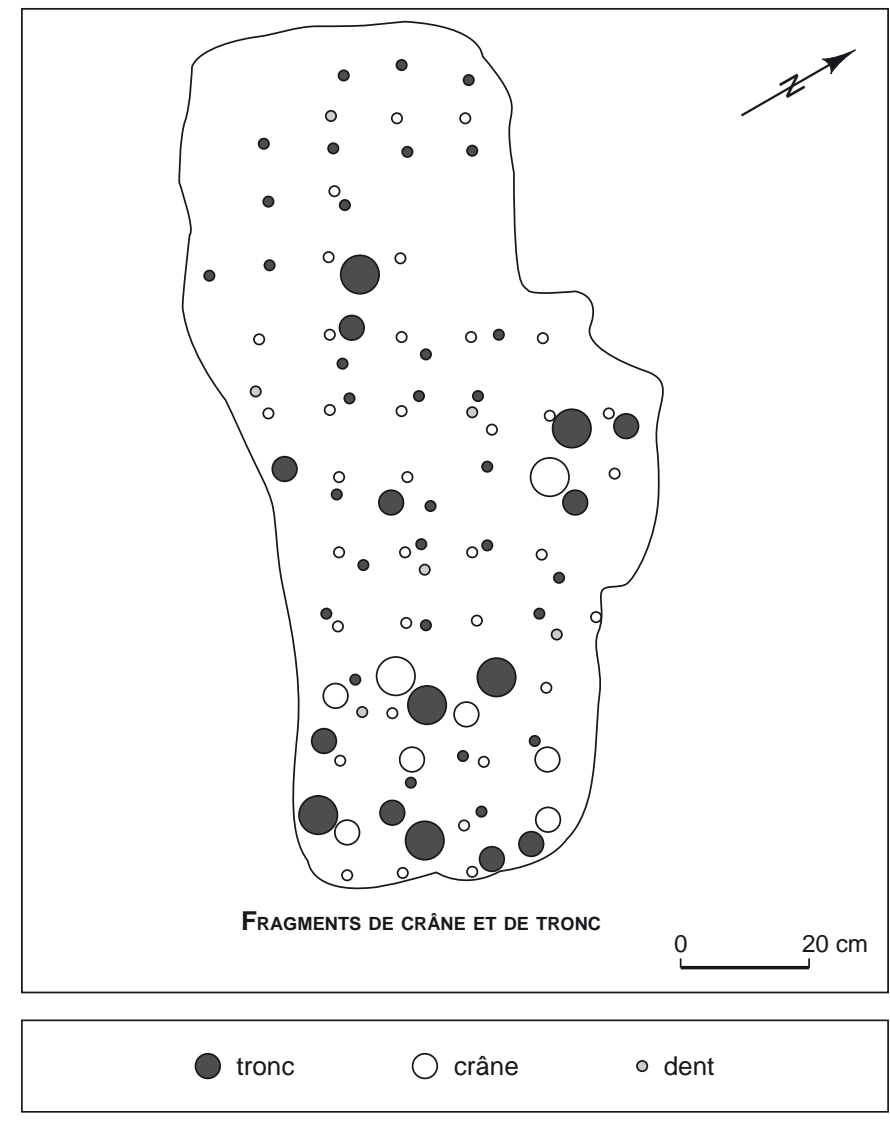

Fig. 104 - Répartition spatiale des régions anatomiques alimentaires dans le bûcher 25 de la Brunerie à Voiron, Isère. La taille des cercles est proportionnelle à la masse de la région anatomique représentée (DAO : F. Blaizot, Inrap).

de la répartition verticale des éléments indique que le corps surmontait le mobilier. Pour la même raison, l'hypothèse d'un ratissage a posteriori de la couche de crémation ne peut pas être retenue.

Une interprétation équivalente a été réalisée par les fouilleurs du bûcher 2 lors du diagnostic du Sextant (rue du Commandant-Charcot à Lyon) daté du $\mathrm{I}^{\mathrm{er}} \mathrm{s}$. de notre ère : en effet, si $78 \%$ de la céramique et $87 \%$ du verre sont concentrés dans la partie orientale de la fosse, $66 \%$ de la masse osseuse se trouve au centre. Toutefois, rien ne permet véritablement d'étayer cette hypothèse ; l'examen des proportions par carré et par couche montre que l'ensemble du mobilier est situé dans la partie est de la fosse, et qu'il se répartit aussi bien dans la couche supérieure des résidus (36 \%) que sur le fond de la fosse. Il apparaît même que la céramique est en majorité confinée dans la couche profonde (91\%), de même que les os humains (94\%), tandis que le verre est à peu près réparti de manière équivalente dans les deux horizons (45\% et $55 \%$ ). Les vestiges du bûcher semblent ainsi plutôt se rapporter globalement au dispositif initial : le corps, qui était celui d'un jeune enfant, fut placé au centre et le mobilier dans la moitié est, la céramique sur le fond et le verre de manière plus étagée. Cet exemple montre combien l'interprétation doit tenir compte du plus grand nombre possible de paramètres.

Dans d'autres bûchers, les remaniements sont moins systématiques et l'on distingue difficilement ce qui relève d'une intervention limitée de l'officiant de ce qui se rapporte à des processus naturels liés aux effets de la combustion ou de l'effondrement du bûcher. L'interprétation se heurte au manque de connaissances théoriques et à l'imprécision des informations disponibles concernant les phénomènes intervenant dans la combustion des corps en contexte de crémation traditionnelle. Ces problèmes d'interprétation ont été longuement développés dans l'étude de la structure de crémation néolithique de ReichstettMundolsheim (Bas-Rhin), qui se caractérise à la fois par le maintien de segments de corps articulés et par une disposition anormale de ces segments les uns par rapport aux autres (Blaizot, 2005). Les exemples des bûchers 5 et 10 de 


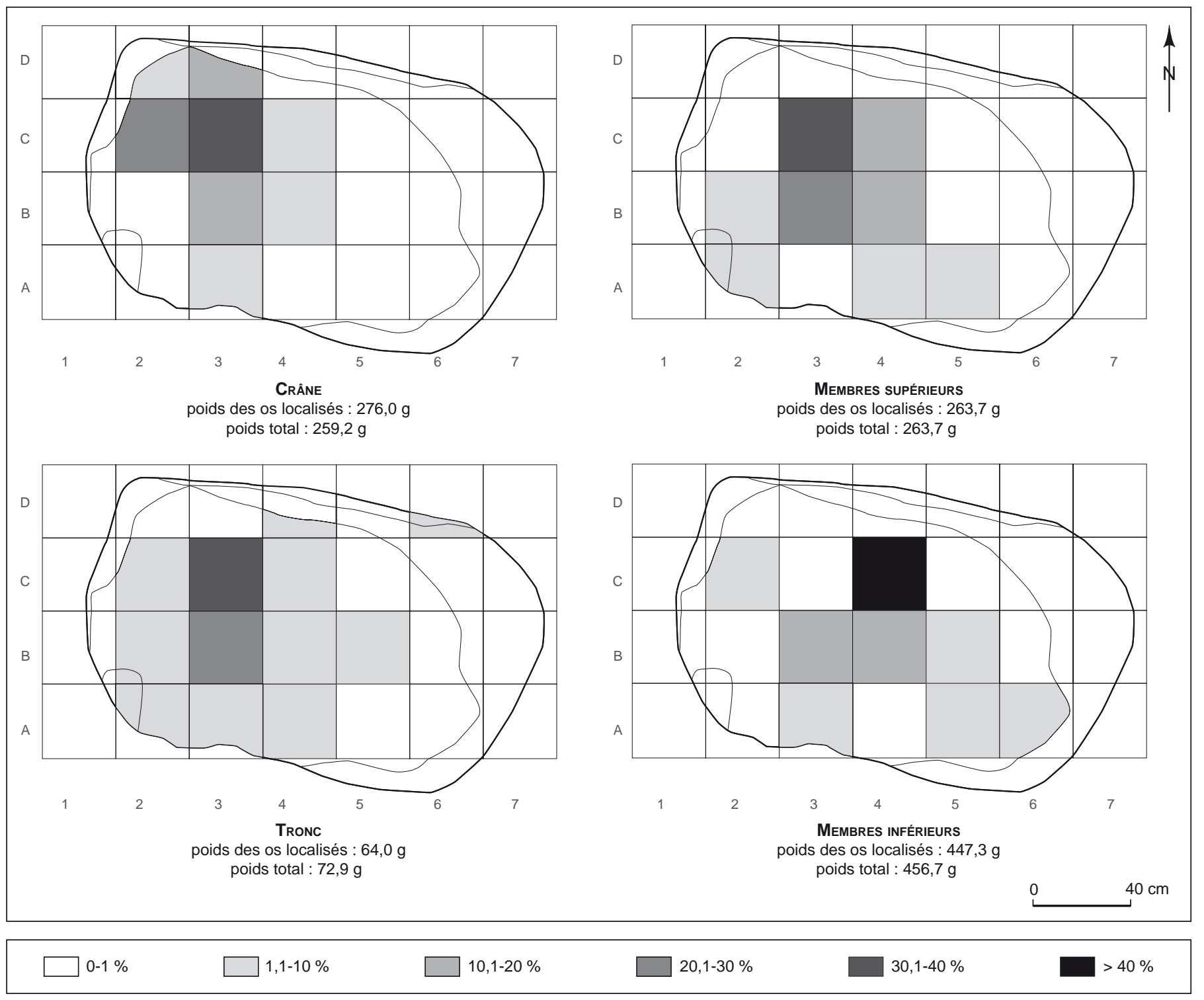

Fig. 105 - Répartition spatiale des restes osseux par régions anatomiques dans le bûcher 10 de l'Estel à Vers-Pont-du-Gard, Gard (relevé : V. Bel, Inrap;DAO : A. Recolin, Inrap).

l'ensemble funéraire de l'Estel à Vers-Pont-du-Gard offrent des cas de figures assez proches.

Dans le bûcher 5 , la distribution spatiale des restes osseux est globalement cohérente et situe la partie supérieure du corps à l'est et la partie inférieure à l'ouest. Toutefois, des remaniements ont concerné les os des jambes, les fémurs, les éléments du tronc, de la base du crâne et des premières cervicales. Ainsi, des fragments de fémur (dont le quart proximal du fémur gauche, $n^{\circ} 17$ ) se trouvent à l'extrémité est, des éléments de rocher et de basi-occipital à l'ouest ( $\left.n^{\circ} 43\right)$, tandis que des fragments d'atlas et d'axis ( $n^{\text {os }} 42$ et
41, en D6-7), sont localisés en aval d'un fragment de sacrum (no 34, en E4) (fig. 101). On observe que les éléments dont la localisation apparaît aberrante sont situés dans la partie supérieure de la couche de cendres, alors que les autres sont au fond. Par ailleurs, un ensemble de pièces en bon rapport anatomique, se rapportant au pied droit ( $\left.\mathrm{n}^{\text {os }} 59,60,79,80\right)$ ont été relevées à l'amont d'un faisceau de diaphyses de fibula, tibia et fémur ( $\left.\mathrm{n}^{\text {os }} 54-55-56\right)$. De la même façon, un autre ensemble de diaphyses de tibia se trouvait en aval d'un groupe d'os en bon rapport appartenant au pied gauche $\left(\mathrm{n}^{\text {os }} 64,65,70\right)$. Cette situation paradoxale des os des 
pieds et des jambes pourrait s'expliquer soit par l'action de l'officiant consistant en une rotation de cette partie du corps dans un geste destiné à ramener les éléments au centre du bûcher, soit en un déplacement « naturel » des pieds. En effet, il a été observé que les extrémités des membres qui comportent un faible volume de chair pouvaient se détacher avant que les contentions ligamentaires ne soient totalement consumées. Si ces parties tombent à la base du bûcher, la combustion peut alors être arrêtée faute d'oxygène et les os du segment sont maintenus en connexion (MacKinley, 1989, p. 72). Une autre hypothèse serait que le sujet était placé sur le ventre : dans ce cas, les genoux se fléchissent et les jambes et les pieds sont rabattus en arrière de la cuisse (sur face postérieure du fémur) sous l'effet de la rétraction des liquides et de la carbonisation musculaire (Grévin, com. pers.). Toutefois, les éléments de membres supérieurs ou de membres inférieurs qui ont été latéralisés suggèrent plutôt que le sujet reposait sur le dos.

Dans le bûcher 10, les restes osseux étaient concentrés au centre de la fosse, et, secondairement, contre les bords nord, ouest et sud, la partie est, étant pratiquement vide d'os. Ce regroupement des éléments laisse toutefois apparaître une distribution inégale des os selon les régions anatomiques (fig. 105). Le plan de répartition des os du crâne est en effet pratiquement le négatif du plan de répartition des os des membres inférieurs. Les premiers sont situés dans le quart nord-ouest, les seconds au sud-est. Les os du tronc et des membres supérieurs sont surtout présents dans la partie médiane. Cette organisation cohérente permet de restituer la position générale du sujet, la tête à l'ouest et les pieds à l'est. Le prélèvement individualisé des principaux fragments a permis de préciser la disposition des pièces osseuses qui, dans certains cas, sont restées en bon rapport anatomique, comme la partie proximale de l'avant-bras gauche ou bien encore les os de la jambe et du pied gauches, conservés en connexion. Contre toute logique anatomique cependant, la partie distale de cet ensemble est située à l'ouest (côté crâne), et la partie proximale à l'est, côté aval. La fibula $\left(n^{\text {os }} 57-58\right)$ et le tibia ( $n^{\text {os }} 55-56$ et 53 ) sont disposés parallèlement aux longs côtés de la fosse, en connexion stricte, en vue postérieure. L'épiphyse proximale du tibia est disjointe et située plus à l'est ( $\left.n^{\circ} 51\right)$. L'épiphyse du tibia ( $\left.n^{\circ} 56\right)$ est en connexion étroite avec le talus ( $\left.n^{\circ} 56\right)$, lui-même en bon rapport avec le calcanéus $\left(\mathrm{n}^{\circ} 63\right)$ et avec le naviculaire et le cuboïde ( $\left.n^{\circ} 59\right)$. La position « anormale » de la jambe et du pied gauche implique un déplacement de cette partie au cours de la crémation. Elle pourrait être attribuée aux effets de la crémation. En effet, les observations effectuées lors de crémations traditionnelles témoignent d'un fléchissement des membres (position du «boxeur » : Grévin, 1997 et 2004 ; Pautreau, Mornais, 2005, p. 49) qui se détachent du tronc. L'officiant aurait ramené la jambe vers le centre du bûcher, en la faisant pivoter à $180^{\circ}$, alors que les contentions ligamentaires, y compris celles de la partie proximale du tarse, étaient maintenues. Il est possible que les os aient été brisés à cet effet à leur extrémité proximale (voir la position du $n^{\circ} 51$ ), comme cela a été observé en Inde par G. Grévin (2004). 